\title{
APLICAÇÃO DO ELEMENTO FINITO DKT À ANÁLISE DE CASCAS
}

ELIAS CALIXTO CARRIJO

Dissertação apresentada à Escola de Engenharia de São Carlos, da Universidade de São Paulo, como parte dos requisitos para obtenção do título de Mestre em Engenharia de Estruturas.

ORIENTADOR : Prof. Dr João Batista De Paiva

São Carlos

1995

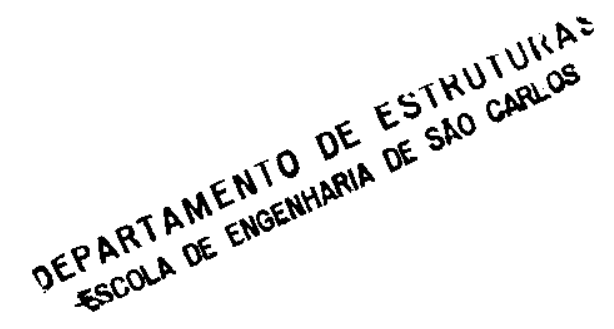


Class.

$\triangle \mathrm{HA}$

tombo

Carrijo, Elias Calixto

C312a Aplicação do elemento finito DKT à análise de cascas / Elias Calixto Carrijo. -- São Carlos, 1995.

89 p.

Dissertação (Mestrado) -- Escola de Engenharia de São CarlosUniversidade de São Paulo, 1995.

Orientador: Prof.Dr. João Batista de Paiva

1. Cascas (Estruturas). 2. Método dos elementos finitos. I. Título. 


\section{AGRADECIMENTOS.}

A DEUS, por tudo.

À Universidade Católica de Goiás, pela licença concedida.

À CAPES, pelo auxilio financeiro.

Ao prof. João B. De Paiva, pela orientação e apoio.

À Karla pela compreensão, amizade e companheirismo nos momentos mais difíceis. 
A meus pais, Jacyra e Onehil. 


\section{SUMÁRIO}

LISTA DE FIGURAS.

LISTA DE TABELAS.

RESUMO.

V

ABSTRACT

vi

1 INTRODUÇÃO.

20 ELEMENTO FINITO DE MEMBRANA . 8

2.1 Introdução. 8

2.2 Relações Básicas Da Teoria Da Elasticidade. 9

2.2.1 Variação Das Tensões. 9

2.2.2 Reciprocidade Das Tensões. 11

2.2.3 Equações De Equilíbrio. 13

2.2.4 Deformações Específicas.

2.2.5 O Estado Plano De Tensões. 19

2.3 Energia Potencial De Um Corpo Elástico. 21

2.4 O Método Dos Elementos Finitos . 22

2.5 Matriz De Rigidez Do Elemento De Membrana. 24

3 O ELEMENTO FINITO DE PLACA . 31

3.1 Introdução. 31

3.2 Hipóteses Básicas Da Placa Solicitada À Flexão. 31

3.3 Condições De Equilibrio De Um Elemento Da Placa. 34 
3.4 Determinação Das Tensões Para Placa Com

Efeito Do Esforço Cortante. 36

3.5 Determinação Dos Esforços. $\quad 42$

3.7 Equação Diferencial Das Placas. $\quad 43$

3.8 Energia De Deformação De Placas Com Efeito

Do Esforço Cortante. 43

3.9 Montagem Da Matriz De Rigidez Do Elemento DKT. 44

4 O ELEMENTO FINITO DE CASCA PLANO. 59

4.1 Introdução. 59

4.2 Rigidez De Um Elemento De Casca Plano Em

Coordenadas Locais. $\quad 59$

4.3 Elemento Finito De Casca Plano. 62

4.4 Rigidez Rotacional Fictícia. $\quad 64$

4.5 Transformação Para Coordenadas Globais. 64

4.5.1 Transformação De Coordenadas Locais Em

Um Sistema Qualquer. $\quad 65$

4.5.2 Montagem Da Matriz De Rigidez No Sistema Global. $\quad 67$

4.5.3 Matriz Dos Cossenos Diretores. 69

5 O PROGRAMA E EXEMPLOS NUMÉRICOS. 72

5.1 Introdução.

5.2 Exemplos.

5.2.1 Exempio. 73

5.2.2 Exemplo. $\quad 75$

5.2.3 Exemplo. $\quad 76$

5.2.4 Exemplo. $\quad 78$ 
6 CONCLUSÃO.

7 BIBLIOGRAFIA. 


\section{LISTA DE FIGURAS}

FIGURA 1.1..Condensação estática do elemento LST.

FIGURA 1.2 Condensação estática do elemento CST.

FIGURA 2.1 Estado de tensões.

9

FIGURA 2.2 Tensões tangenciais.

FIGURA 2.3 Corpo sob ação de carregamento.

FIGURA 2.4 Paralelepípedo infinitesimal.

FIGURA 2.5 Projeção dos deslocamentos..

FIGURA 2.6 Chapa sob ação de carregamento.

FIGURA 2.7 Coordenadas adimensionais.

FIGURA 2.8 Elemento CST.

FIGURA 3.1 Placa sob carregamento. 
FIGURA 3.3 Relações geométricas no elemento triangular.

FIGURA 4.1 Elemento finito plano.

60

FIGURA 4.2 Elemento finito de casca plano.

63

FIGURA 4.3 Sistema global e local.

68

FIGURA 4.4 Casca dividida em elementos triangulares planos.

69

FIGURA 5.1 Viga engastada com carga concentrada na extremidade.

FIGURA 5.2 Viga engastada inclinada no espaço.

FIGURA 5.3 Viga engastada inclinada no espaço.

FIGURA 5.4 Comportamento do modelo para viga engastada . 76

FIGURA 5.5 Placa inclinada engastada nas quatro bordas .

FIGURA 5.6 Comportamento da placa inclinada no espaço.

FIGURA 5.7 Cobertura em casca cilindrica.

FIGURA 5.8 Comportamento do modelo para momento e deslocamento transversal no ponto $\mathrm{C}$. 
FIGURA 5.9 Comparação entre a solução analitica e a execução da malha $14 \times 20$, do deslocamento transversal na seção central.

FIGURA 5.10 Comparação entre a solução analítica e a execução da malha $14 \times 20$ para o deslocamento longitudinal no apoio .

FIGURA 5.11 Comparação entre a solução analitica e a malha $14 \times 20$ do momento transversal na seção central. 81 
LISTA DE TABELAS

TABELA 5.1 Deslocamentos na viga engastada (sistema global) 
RESUMO

CARRIJO, E. C. Aplicação do elemento finito DKT à análise de cascas. São Carlos, 1995. 87p Dissertação (Mestrado)- Escola de Engenharia de São Carlos, Universidade de São Paulo.

Neste trabalho apresenta-se um elemento finito de casca plano que é obtido pela composição de um elemento de placa com um de membrana. $O$ elemento de placa usado, o DKT( Discrete Kirchhoff Theory), pertence à classe dos elementos triangulares com nove graus de liberdade( uma translação e duas rotações por nó), e é obtido pela imposição da hipótese de Kirchhoff nos seus pontos nodais. Para o elemento de membrana, usou-se o elemento CST( Constant Strain Triangle), com seis graus de liberdade( duas translaçōes por nó). O elemento finito resultante DCT, possui dezoito graus de liberdade ( tres translaçōes e tres rotaçōes, sendo uma das rotações fictícia- no plano do elemento). Simples exemplos de placa e chapa no espaço foram analisados para se testar o elemento, e finalmente uma casca cilindrica uniformemente carregada foi analisada e o resultado obtido comparado com o fornecido por outros autores. Neste estudo, o elemento DKT mostrouse eficiente na análise de cascas.

Palavras-chave : Elementos finitos, casca, plano, placa, membrana, DKT, CST, DCT. 
ABSTRACT

CARRIJO, E. C. DKT finite element aplication in shells analysis. São Carlos, 1995. 87p Dissertação (Mestrado)- Escola de Engenharia de São Carlos, Universidade de São Paulo.

This work presents a flat shell finite element composed of plates in bending and in tension finite elements. The bending element used, the DKT ( Discrete Kirchhoff Theory) belongs to the class of triangular elements with nine degrees of freedom( the transverse displacement and its derivatives at each node) and it is obtained from the theory of plates with shear force influence with Kirchhoff hypothesis imposed in the nodes of the element. The tension finite element is the well known CST( Constant Strain Triangle) with six degrees of freedom ( the displacement $u$ and $v$ at each node). The resulting finite element, called DCT, has 18 degrees of freedom, including an extra and fictitious rotation in the normal direction to rhe surface of elements. Single plates in bending and in tension described in a tridimensional system of coordinates were analyzed to test the adopted finite elements and finally cylindrical shell uniformly loaded were analyzed and the results compared with those of authors. In the work the DKT element shown to be a reliable finite element to be used in shell analysis.

Keywords: Finite elements, shells, flat, plate, membrane, DKT, CST,DCT. 


\section{INTRODUÇÃO}

Uma casca é essencialmente uma estrutura que se pode obter a partir de uma placa, transformando o plano médio em uma superfície curva. O estudo de seu comportamento tem sido objeto de intensa pesquisa na Engenharia de Estruturas. Mesmo sendo válidas as mesmas hipóteses da teoria de placas com relação à distribuição transversal de deformações e tensões, a forma com que as cascas resistem às cargas externas é diferente de uma placa plana. Em FLUGGE (1960) tem-se um trabaho inicial sobre o comportamento de cascas. Também em BILLINGTON(1982), encontra-se um estudo do comportamento das cascas pela teoria clássica.

No caso de uma casca, a obtenção detalhada das equações que descrevem o seu comportamento é extenso e complexo. Mesmo com toda pesquisa matemática envolvendo a solução analítica de equações diferenciais, nem sempre é possível encontrar soluçōes para esse tipo de equação.

Entretanto, com o surgimento dos computadores, o uso de métodos numéricos para a resolução dessas equaçōes sofreu grande avanço, apresentando bom grau de confiabilidade.

Dentre os vários métodos conhecidos, o Método dos Elementos Finitos ( MEF ), tem merecido grande atenção dos pesquisadores de diversas áreas pela sua versatilidade. 
O MEF baseia-se na divisão de domínios em subregiões, a partir das quais pode-se obter o comportamento de toda estrutura a ser analisada. 0 seu inicio foi com COURANT (1943), ao estudar a torção de Saint-Venant aplicando princípios variacionais.

O Método dos Elementos Finitos, como é conhecido hoje, foi proposto por TURNER et al (1956), ao estender o estudo apresentado por COURANT (1943) e ARGYRIS( 1950) ao comportamento de estruturas bidimensionais. O termo "Elementos Finitos" foi usado pela primeira vez por CLOUGH ( 1960).

A partir de então a pesquisa sobre o MEF tem sido intensa, somando em 1986 mais de 20.000 artigos sobre o mesmo (COOK,1989).

Na verdade, o MEF é uma poderosa ferramenta não só no estudo de estruturas espaciais, mas de diversos problemas, desde o comportamento de estruturas, até a análise de fenômenos hidráulicos.

Para se analisar cascas pelo Método dos Elementos Finitos, pode-se ter elementos finitos curvos e planos.

Os elementos curvos se adaptam à forma da casca em questão. Estabelecendo-se relações entre coordenadas curvilíneas e cartesianas, pode-se chegar na montagem da matriz de rigidez desses elementos.

A idéia de empregar as funções de forma do elemento para estabelecer coordenada curvilíneas foi proposta inicialmente por TAIG(1961), estabelecendo relações básicas para um quadrilátero. Posteriormente IRONS(1966) generalizou a proposta para outros elementos. Tais elementos tem como principal característica a rápida convergência para - resultado teórico, como pode ser verificado em recente trabalho apresentado por BHIMARADDI(1989), onde apresenta-se um elemento isoparamétrico quadrangualar com 64 graus de liberdade, incluindo o efeito do esforço cortante, para se analisar cascas de revolução.

Entretanto, mesmo com excelentes resultados, por ser baseada na teoria clássica de cascas, sua formulação é complexa, além de dificil associação com elementos de viga, o que torna sua aplicação restritiva. 
Inicialmente proposto por CLOUGH E JOHNSON (1968), os elementos de casca planos resultam do acoplamento entre um elemento de placa e outro de membrana, subdividindo a casca em várias regiões planas,. Além de bons resultados, apresentam uma formulação facilitada pelo simples acoplamento entre um elemento de placa e outro de membrana.

Os resultados desses elementos podem ser verificados nos trabalhos de DHATT(1986), onde se apresenta um elemento de casca a partir do elemento DKTP (Discrete Kirchhoff Theory Plus), acoplado ao elemento de membrana com variação quadrática para a deformação, o LST( Linear Strain Triangle). IBRAHIMBEGOVIC (1990) analisa um elemento de casca quadrangular, a partir do qual se gera um elemento triangular pela junção de dois de seus nós. PHAAL E CALLADINE (1992-11) obtém um elemento de casca plano a partir dos elementos HSB (Hinged Shell Bending) e CST (Constant Strain Triangle), e ALLMAN (1993) propõe um novo elemento de casca com tres pontos nodais e seis graus de liberdade por nó, no qual os elementos de membrana e placa possuem variação cúbica para seus respectivos deslocamentos.

HO (1992), faz uma comparação do desempenho entre os elementos plano( cujo elemento de membrana tem variação constante da tensão ao longo de sua face) e curvo ( superparamétrico quadrangular com oito pontos), mostrando em quais situações esses elementos tem bom comportamento.

Apesar de não convergirem tão rápido quanto os elementos curvos,os elementos planos tem fornecido resultados satisfatórios na análise de cascas, como pode ser verificado nos trabalhos já citados.

Neste trabalho procurou-se formular um elemento de casca plano com bons resultados, cuja formulação não demandasse cálculos extenuantes. Portanto ter-se-ia que buscar entre os elementos de placa e membrana aqueles que melhor se adaptassem.

Entre os elementos de placa, tem merecido atenção especial os elementos triangulares com nove graus de liberdade. Comparando os 
diversos elementos pertecentes à essa classe, BATOZ (1980) destaca os elementos DKT ( Discrete Kirchhoff Teory) e HSM( Hybrid Stress Metod) como os mais eficientes dentre os estudados na análise de placas delgadas. Este estudo comparou os resultados do elemento já mencionado com os seguintes elementos: HCT( CLOUGH, 1968), A, T e T-10 (CLOUGH, 1965), SRI (Seletive Reduced Integration), BCIZ1 e BCIZ2(BAZELEY et alli, 1965), além do elemento CPT usado no programa ICES-STRUDLII.

Baseados na generalização da hipótese de Kirchhoff, BATOZ E LARDEUR (1989) apresentam um novo, com nove graus de liberdade elemento para análise de placa espessas, o DST( Discrete Shear Triangle). Entretanto, os resultados para placas delgadas são os mesmos obtidos por BATOZ (1980).

Pode-se lançar mão, também, de elementos com mais de tres pontos nodais, na análise de estruturas pelo método dos elementos finitos. Entretanto surge um problema inerente da formulação desse elemento, que é a dimensão da largura da semi-banda, que obriga a ocupar grande espaço na memória volátil do computador.

Apesar de recentes pesquisas com o intuito de se encontrar um elemento com nove graus de liberdade com melhores resultados, como pode ser visto em PETROLITO (1989) (onde se apresenta os resultados do elemento ACM modificado para placas espessas), e em PHAAL E CALLADINE (1992) (onde se analisa o comportamento do elemento HPB (Hinged Plate Bending), tem-se no DKT ainda uma excelente opção na análise de placas delgadas.

Assim, para se obter um elemento de casca, necessita-se de um elemento de membrana que possa se associar a um dos elementos de placa.

Para os elementos de membrana pode-se fazer uso de um dos elementos conhecidos na bibliografia corrente, o caso dos elementos triangulares CST ( Constant Strain Triangle), LST (Linear Strain Triangle) e QST ( Quadratic Strain Triangle). Ou dos elementos quadrangulares 
isoparamétricos com quatro, oito e doze pontos nodais. Formulações mais recentes para elementos de membrana podem ser encontadas nos trabalhos de IBRAHIMBEGOVIC et alli( 1990), também apresenta um elemento quadrangular com rotações independentes. SALMON e ABEL (1989) analisam o comportamento do elemento Q9 para as situações às quais não se tem bons resultados. YUAN et alli ( 1993) apresentam uma nova formulação para o elemento de membrana híbrido com quatro pontos nodais, a partir de uma rotação no sistema de referência das coordenadas isoparamétricas.

No presente trabalho, optou-se por encontrar um elemento ao qual fosse possivel o acoplamento com o elemento DKT, motivado pelo exfecelente comportamento desse último.

Utilizando-se da condensação estática proposta por WILSON (1974), CODA ( 1993) propõe a formação de um elemento finito de membrana, a partir do elemento LST, eliminando-se os pontos nodais intermediários nos lados do elemento, conforme figura a seguir.

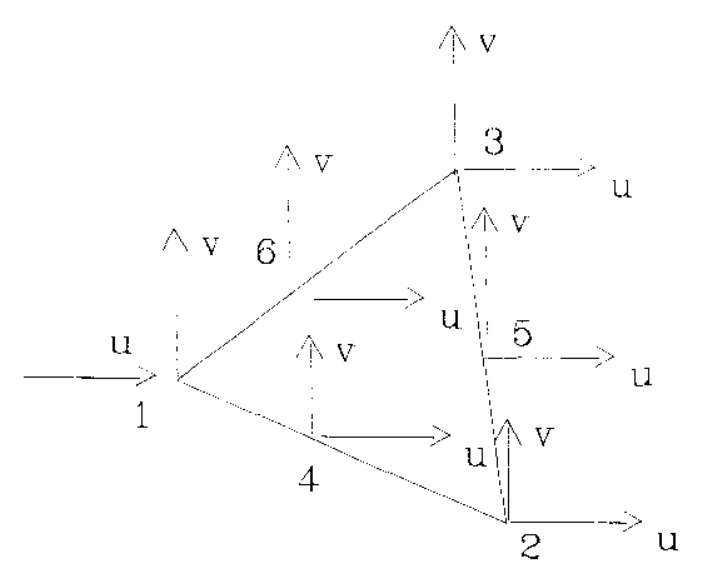

LST condensacao

estatica

Figura 1.1 Condensação estática do elemento LST. 
Apesar dos resultados obtidos para o processo dinâmico serem satisfatórios (CODA ,1993), para a análise estática não se obteve resultados confiáveis, impossibilitando o aproveitamento desse elemento.

Seguindo o mesmo procedimento da condensação estática, tentou-se um elemento que pudesse ser obtido a partir da combinação de tres elementos CST, conforme figura que segue.

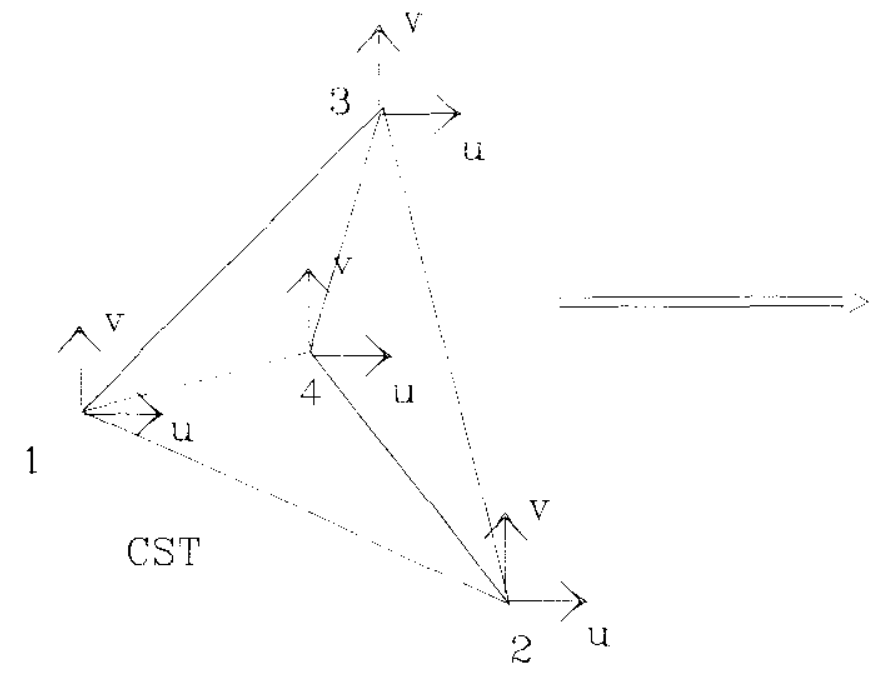

Figura 1.2 Condensação estática do elemento CST.

Entretanto, não houve meihora no resultado que motivasse a opção por esse elemento. Na verdade, verificou-se a completa identidade de resultados.

Apesar de não ser o elemento com melhores resultados, o CST oferece a vantagem de se acoplar facilmente com o DKT, com resultados comprovadamente eficientes. Os resultados obtidos serão comentados no fim desse trabalho. 
Apresenta-se então a base teórica que leva à montagem desse elemento de casca plano. No capítulo II apresenta-se o elemento finito de membrana. É feito uma breve introdução aos conceitos da teoria da elasticidade. Em seguida desenvolve-se a matriz de rigidez desse elemento, baseando-se no princípio da mínima energia potencial.

O elemento finito de placa ( DKT), é apresentado no capítulo III, explicitando-se todos os detalhes que levam a obtenção da matriz de rigidez do mesmo.

O processo de montagem da matriz de rigidez do elemento finito de casca plano está exposto no capítulo IV.

Os resultados obtidos são apresentados no capítulo $V$. Os comentários com relação aos resultados e sugestōes para prosseguimento da pesquisa são feitos no capitulo $\mathrm{VI}$, onde se apresentam as conclusões finais. 


\section{O ELEMENTO FINITO DE MEMBRANA}

\subsection{INTRODUÇÃO}

Como já foi dito anteriormente, para se montar um elemento finito de casca plano, necessita-se de um elemento finito de placa e outro de membrana. Nesse capítulo desenvolve-se o elemento finito de membrana que será usado na montagem desse elemento de casca. Os esforços devidos ao efeito de membrana são obtidos a partir desse elemento.

O elemento finito de membrana usado nesse trabalho é o CST( Constant Strain Triangle). Nesse elemento supõe-se que as deformaçōes ocorridas na face do mesmo sejam constantes. Todo o desenvolvimento para se chegar na matriz de rigidez desse elemento será exposto a seguir. Antes, porém, se fará uma breve recordação dos conceitos da teoria da elasticidade, que são essenciais nesse desenvolvimento.

As hipóteses básicas adotadas são:

a. O material é elástico-linear e obedece a lei de Hooke;

b. O material é homogêneo e isótropo. 


\subsection{RELAÇÕES BÁSICAS DA TEORIA DA ELASTICIDADE}

\subsubsection{VARIAÇÃO DAS TENSÕES.}

No interior de um sistema elástico as tensões variam de ponto para ponto, em função das coordenadas $x, y$ e $z$ dos pontos estudados. Considere agora, o paralelepípedo infinitesimal da figura 2.1:

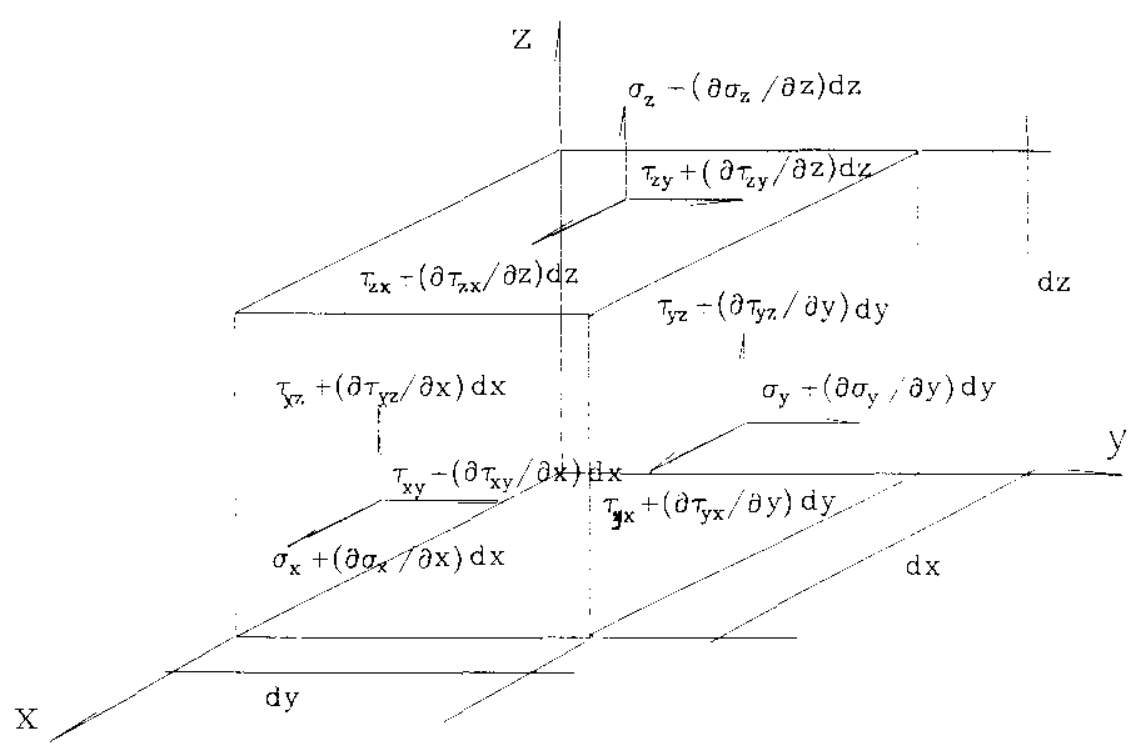

Figura 2.1 Estado de tensões.

Neste paralelepípedo, são dadas as tensões nas faces definidas pelos planos coordenados e se deseja obter as tensões em faces situadas em planos distantes de $d x, d y$ e $d z$, respectivamente. No corpo indicado na figura 2.1 estão indicados os sentidos positivos das tensões nas seis faces 
do paralelepípedo. Nesta figura ainda estão indicadas as forças por unidade de volume, $X, Y$ e $Z$. Conhecendo-se as tensões nos planos coordenados, pode-se conhecê-las em qualquer outro plano, bastando para isso aplicar a fórmula de Taylor. Assim, para a tensão $\sigma_{x}$, na face oposta ao plano yz, distante de $\mathrm{dx}$ tem-se:

$$
\sigma_{x}+\left(\frac{\partial \sigma_{x}}{\partial x}\right) d x+\left(\frac{1}{2 !}\right)\left(\frac{\partial^{2} \sigma_{x}}{\partial^{2} x}\right)(d x)^{2}+\ldots
$$

O mesmo acontecendo para as demais tensões situadas no plano yz, ou seja:

$$
\begin{aligned}
& \tau_{x y}+\left(\frac{\partial \tau_{x y}}{\partial x}\right) d x+\left(\frac{1}{2 !}\right)\left(\frac{\partial^{2} \tau_{x y}}{\partial x^{2}}\right)(d x)^{2}+\ldots \\
& \tau_{x z}+\left(\frac{\partial \tau_{x z}}{\partial x}\right) d x+\left(\frac{1}{2 !}\right)\left(\frac{\partial^{2} \tau_{x z}}{\partial x^{2}}\right)(d x)^{2}+\ldots
\end{aligned}
$$

Nas expressões (2.1), (2.2) e (2.3), pode-se desprezar os termos $(d x)^{2},(d x)^{3}, \ldots$, em presença dos dois primeiros, resultando:

$$
\begin{aligned}
& \sigma_{x}+\left(\frac{\partial \sigma_{x}}{\partial x}\right) d x \\
& \tau_{x y}+\left(\frac{\partial \tau_{x y}}{\partial x}\right) d x \\
& \tau_{x z}+\left(\frac{\partial \tau_{x z}}{\partial x}\right) d x
\end{aligned}
$$


Procedendo da mesma forma com os demais planos coordenados, obtém-se para o plano xz:

$$
\begin{gathered}
\sigma_{y}+\left(\frac{\partial \sigma_{y}}{\partial y}\right) d y \\
\tau_{x y}+\left(\frac{\partial \tau_{x y}}{\partial y}\right) d y \\
\tau_{y z}+\left(\frac{\partial \tau_{y z}}{\partial y}\right) d y
\end{gathered}
$$

E para o plano $x y$ :

$$
\begin{aligned}
& \sigma_{z}+\left(\frac{\partial \sigma_{z}}{\partial z}\right) d z \\
& \tau_{z x}+\left(\frac{\partial \tau_{z x}}{\partial z}\right) d z \\
& \tau_{z y}+\left(\frac{\partial \tau_{z y}}{\partial z}\right) d z
\end{aligned}
$$

\subsubsection{RECIPROCIDADE DAS TENSÕES TANGENCIAIS.}

O corpo analisado obedece as equações de equilibrio da estática, ou seja, a somatória das forças nas direções $\mathrm{X}, \mathrm{Y}$ e $\mathrm{Z}$ e dos momentos em 
relação aos eixos devem ser nulos. Assim, calculando-se o momento em relação ao eixo z'( localizado no centro do retangulo) obtém-se :

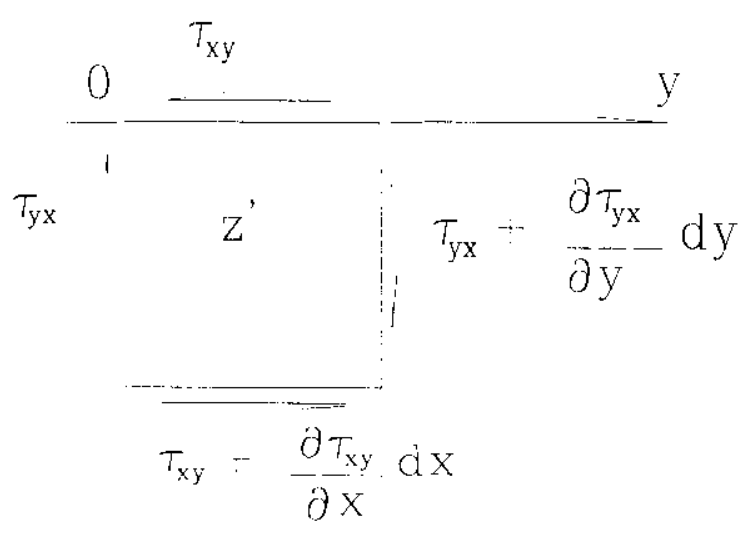

$\mathrm{x}$

Figura 2.2 Tensōes tangenciais.

$$
\Sigma \mathrm{M}_{\mathrm{z}}=0 \quad \Rightarrow
$$

$$
\begin{array}{r}
\tau_{y x}(d x d z)\left(\frac{d y}{2}\right)+\left(\tau_{x y}+\left(\frac{\partial \tau_{x y}}{\partial y}\right) d y\right) d x d z\left(\frac{d y}{2}\right)-\tau_{x y} d y d z\left(\frac{d x}{2}\right)- \\
\left(\tau_{x y}+\left(\frac{\partial \tau_{x y}}{\partial x}\right) d x\right) d y d z\left(\frac{d x}{2}\right)=0
\end{array}
$$

Desprezando-se os infinitésimos de ordem superior e simplificando-se, vem:

$$
\tau_{x y}=\tau_{y x}
$$


Utilizando-se o mesmo procedimento para os eixo $x$ e $y$, indicados na figura 2.2 obtém-se :

$$
\begin{aligned}
& \tau_{x z}=\tau_{z x} \\
& \tau_{y z}=\tau_{z y}
\end{aligned}
$$

\subsubsection{EQUAÇÕES DE EQUILÍBRIO.}

As condições de equilibrio nas direções $X, Y$ e $Z$ são dadas por :

$$
\begin{aligned}
& \Sigma \mathrm{F}_{\mathrm{x}}=0 \\
& \Sigma \mathrm{F}_{\mathrm{y}}=0 \\
& \Sigma \mathrm{F}_{\mathrm{z}}=0
\end{aligned}
$$

Assim, para direção X (figura 2.1) :

$$
\begin{aligned}
& -\sigma_{x} d y d z+\left(\sigma_{x}+\left(\frac{\partial \sigma_{x}}{\partial x}\right) d x\right) d y d z-\tau_{y x} d x d z+\left(\tau_{y x}+\left(\frac{\partial \tau_{y x}}{\partial y}\right) d y\right) d x d z- \\
& -\tau_{z x} d x d y+\left(\tau_{z x}+\left(\frac{\partial \tau_{z x}}{\partial z}\right) d z\right) d x d y+X d x d y d z=0
\end{aligned}
$$

Simplificando e dividindo por $d x . d y . d z$ resulta em:

$$
\frac{\partial \sigma_{x}}{\partial \mathrm{x}}+\frac{\partial \tau_{y x}}{\partial y}+\frac{\partial \tau_{z x}}{\partial z}+X=0
$$

Analogamente para as direções $Y$ e $Z$ obtém-se, respectivamente : 


$$
\frac{\partial \sigma_{y}}{\partial y}+\frac{\partial \tau_{z y}}{\partial z}+\frac{\partial \tau_{x y}}{\partial x}+Y=0
$$

e,

$$
\frac{\partial \sigma_{z}}{\partial z}+\frac{\partial \tau_{x z}}{\partial x}+\frac{\partial \tau_{y z}}{\partial y}+Z=0
$$

\subsubsection{DEFORMAÇÕES ESPECÍFICAS.}

Considere um corpo elástico qualquer, em equilibrio, e um ponto $P$ no seu interior, conforme mostra a figura 2.3 :

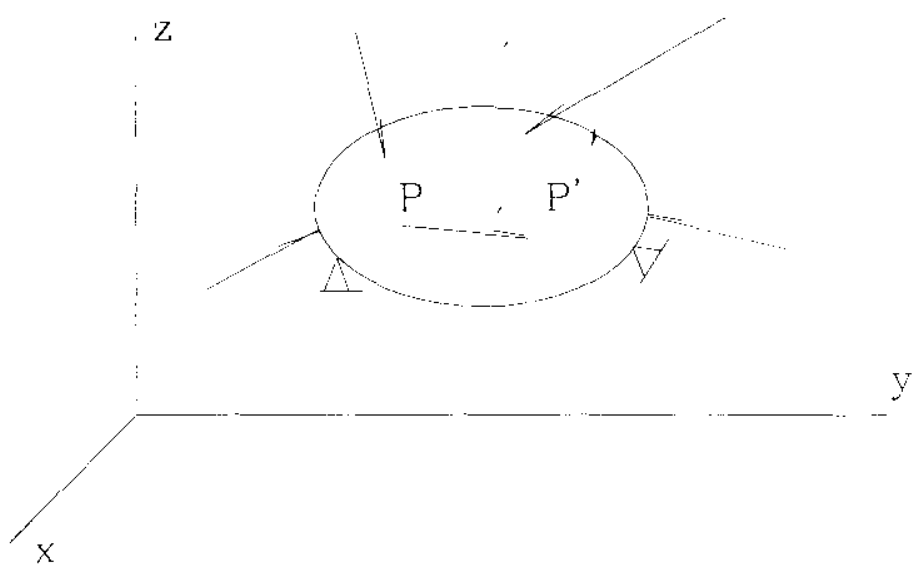

Figura 2.3 Corpo sob ação carregamento.

Pela ação do carregamento, o ponto $P$ sofre um deslocamento vindo a ocupar a posição $\mathrm{P}^{\prime}$. O deslocamento $P P^{\prime}$ é uma função das variáveis $x, y$ e z. Também as componentes deste deslocamento, segundo os eixos $x, y e$ $z$ denominados respectivamente $u, v$ e $w$, são também funções dessas variáveis, isto é: 


$$
\begin{aligned}
& u=u(x, y, z) \\
& v=v(x, y, z) \\
& w=w(x, y, z)
\end{aligned}
$$

Seja agora um paralelepípedo infinitesimal, de lados $d x$, dy e $d z$, como mostra a figura 2.4 :

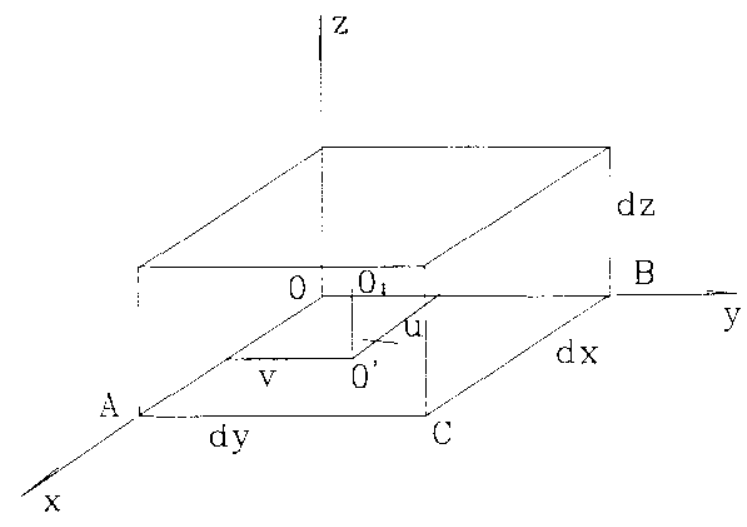

Figura 2.4 Paralelepipedo infinitesimal.

Após o carregamento o ponto $O$ passa a ocupar a posição $O_{1}$, cuja projeção sobre o plano xy representa-se por $\mathrm{O}^{\prime}$. Na figura 2.5 está a ampliação do que ocorre no plano $x y$. $O$ ponto $O$ sofre os deslocamentos u $\mathrm{e}$ $v$, enquanto os lados $O A$ e OB, passam a ocupar as posições O'A"' e O'B"', como mostra a figura 2.5 . 


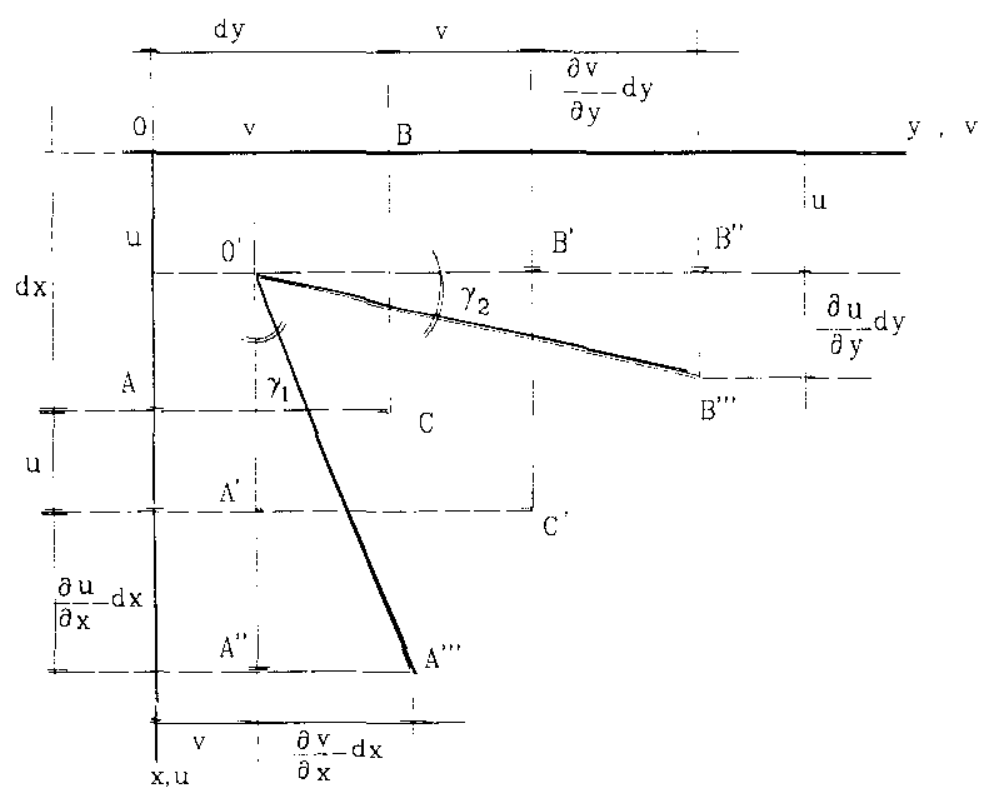

Figura 2.5 Projeção dos deslocamentos.

A deformação especifica na direção $x$ é dada por

$$
\varepsilon_{x}=\frac{\left(\frac{\partial u}{\partial x}\right)(d x)}{d x}=\left(\frac{\partial u}{\partial x}\right)
$$

Da mesma forma :

$$
\begin{aligned}
& \varepsilon_{\mathrm{y}}=\frac{\partial \mathrm{v}}{\partial \mathrm{y}} \\
& \varepsilon_{\mathrm{z}}=\frac{\partial \mathrm{w}}{\partial \mathrm{z}}
\end{aligned}
$$

A distorção angular do lado OA é dada por :

$$
\operatorname{tg} \gamma_{1}=\partial v / \partial x
$$


Como as deformações são muito pequenas é válida a relação:

$$
\gamma_{1}=\partial v / \partial x
$$

Analogamente, para o lado OB:

$$
\gamma_{2}=\partial u / \partial y
$$

Portanto, a distorção angular do elemento considerado é:

$$
\gamma_{\mathrm{xy}}=\gamma_{1}+\gamma_{2}=\frac{\partial \mathrm{v}}{\partial \mathrm{x}}+\frac{\partial \mathrm{u}}{\partial \mathrm{y}}
$$

Para os outros planos tem-se :

$$
\begin{aligned}
& \gamma_{x z}=\frac{\partial w}{\partial x}+\frac{\partial u}{\partial z} \\
& e \\
& \gamma_{y z}=\frac{\partial w}{\partial y}+\frac{\partial v}{\partial z}
\end{aligned}
$$

As relações tensão-deformação podem ser expressas na seguinte forma matricial :

$$
\varepsilon_{\mathrm{r}}=\mathbf{N} \mathbf{u}_{\mathrm{r}}
$$


onde :

$$
\varepsilon_{\mathrm{r}}=\left\{\begin{array}{c}
\varepsilon_{\mathrm{x}} \\
\varepsilon_{\mathrm{y}} \\
\varepsilon_{\mathrm{z}} \\
\gamma_{\mathrm{xy}} \\
\gamma_{\mathrm{xz}} \\
\gamma_{\mathrm{yz}}
\end{array}\right\}
$$$$
\mathbf{u}_{\mathrm{r}}=\left\{\begin{array}{c}
\mathrm{u} \\
\mathrm{v} \\
\mathrm{w}
\end{array}\right\}
$$

$$
\mathbf{N}=\left[\begin{array}{ccc}
\partial / \partial \mathrm{x} & 0 & 0 \\
0 & \partial / \partial \mathrm{y} & 0 \\
0 & 0 & \partial / \partial \mathrm{z} \\
\partial / \partial \mathrm{y} & \partial / \partial \mathrm{x} & 0 \\
\partial / \partial \mathrm{z} & 0 & \partial / \partial \mathrm{x} \\
0 & \partial / \partial \mathrm{z} & \partial / \partial \mathrm{y}
\end{array}\right]
$$

Como já foi dito, o material obedece a lei de Hooke, isto é:

$$
\begin{aligned}
& \varepsilon_{x}=\frac{1}{E}\left[\sigma_{x}-v\left(\sigma_{y}+\sigma_{z}\right)\right]=\frac{\partial u}{\partial x} \\
& \varepsilon_{y}=\frac{1}{E}\left[\sigma_{y}-v\left(\sigma_{x}+\sigma_{z}\right)\right]=\frac{\partial v}{\partial y} \\
& \varepsilon_{z}=\frac{1}{E}\left[\sigma_{z}-v\left(\sigma_{x}+\sigma_{y}\right)\right]=\frac{\partial w}{\partial z} \\
& \gamma_{x y}=\frac{1}{G} \tau_{x y}=\frac{\partial v}{\partial x}+\frac{\partial u}{\partial y} \\
& \gamma_{x z}=\frac{1}{G} \tau_{x z}=\frac{\partial u}{\partial z}+\frac{\partial w}{\partial x} \\
& \gamma_{y z}=\frac{1}{G} \tau_{y z}=\frac{\partial v}{\partial z}+\frac{\partial w}{\partial y}
\end{aligned}
$$

Onde: $\mathrm{E}$ módulo de Young

$\checkmark$ Coeficiente de Poisson

$G=E /(2(1+v))$ 


\subsubsection{O ESTADO PLANO DE TENSÕES}

A análise dos problemas elásticos é extremamente simplificada quando as tensões, ou deslocamentos, processam-se sempre paralelamente a um determinado plano. Na prática, grande número de problemas podem ser reduzidos a estes casos, isto é, a um estado de tensões ou deformações planas .

Seja uma membrana, como mostra a figura 2.6a, sendo a figura $2.6 \mathrm{~b}$ uma representação do corte transversal dessa chapa. Admite-se que a espessura $t$ é muito pequena em relação às outras dimensões. As forças que mantém o corpo em equilibrio atuam paralelamente ao plano médio da chapa, como mostra a figura $2.6 \mathrm{~b}$. Se não houverem forças externas atuando nas faces da chapa, a tensão $\sigma_{z}$ será nula. O mesmo acontecerá com as tensões tangenciais $\tau_{x z}$ e $\tau_{y z}$. Admitindo que isso ocorra em todos os planos paralelos às faces da chapa, tem-se definido um estado plano de tensões. O erro desta hipótese será tanto menor quanto menor for a espessura t. Assim, na lei de Hooke, fazendo-se $\sigma_{z}=\tau_{x z}=\tau_{y z}=0$, ter-se-á:

$$
\begin{aligned}
& \varepsilon_{x}=(1 / E)\left[\sigma_{x}-v \sigma_{y}\right] \\
& \varepsilon_{y=}(1 / E)\left[\sigma_{y}-v \sigma_{x}\right] \\
& \gamma_{x y}=(1 / E)(2(1+v)) \tau_{x y}
\end{aligned}
$$

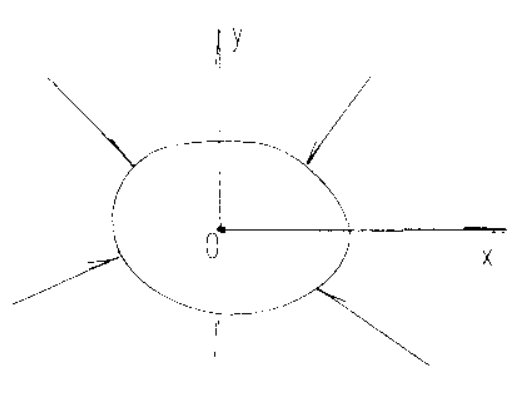

(a)

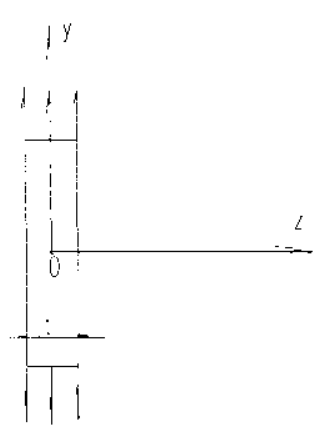

(b)

Figura 2.6 Chapa sob ação de carregamento. 
Estas equações podem ser expressas na forma matricial como se segue :

$$
\varepsilon=\text { C. } \sigma
$$

onde:

$$
\begin{aligned}
& \varepsilon=\left\{\begin{array}{c}
\varepsilon_{\mathrm{x}} \\
\varepsilon_{\mathrm{y}} \\
\gamma_{\mathrm{xy}}
\end{array}\right\} \quad \text { e, } \quad \sigma=\left\{\begin{array}{c}
\sigma_{\mathrm{x}} \\
\sigma_{\mathrm{y}} \\
\tau_{\mathrm{xy}}
\end{array}\right\} \\
& \mathbf{C}=\frac{1}{\mathrm{E}}\left[\begin{array}{ccc}
1 & -v & 0 \\
-v & 1 & 0 \\
0 & 0 & 2(1+v)
\end{array}\right]
\end{aligned}
$$

Para expressar as tensões em função das deformações, basta inverter a equação (2.36) :

$$
\sigma=\mathrm{D} \varepsilon
$$

onde:

$$
\mathbf{D}=\frac{E}{1-v^{2}}\left[\begin{array}{ccc}
1 & v & 0 \\
v & 1 & 0 \\
0 & 0 & \frac{1-v}{2}
\end{array}\right]
$$


Nesse estado plano de tensões, as deformações são expressas como segue :

$$
\varepsilon=\mathbf{L} . \mathbf{u}
$$

onde :

$$
\mathbf{u}=\left\{\begin{array}{l}
u \\
v
\end{array}\right\} \quad \text { e } \quad \mathbf{L}=\left[\begin{array}{cc}
\frac{\partial}{\partial x} & 0 \\
0 & \frac{\partial}{\partial y} \\
\frac{\partial}{\partial y} & \frac{\partial}{\partial x}
\end{array}\right]
$$

\subsection{ENERGIA POTENCIAL DE UM CORPO ELÁSTICO}

Dado um campo de deslocamentos qualquer :

$$
\begin{aligned}
& u=u(x, y, z) \\
& v=v(x, y, z) \\
& w=w(x, y, z)
\end{aligned}
$$

que satisfaz as condições de contorno do problema, então se diz que este é um campo de deslocamentos admissiveis. A partir deste campo é possível calcular as deformações específicas $e$ as tensões. Com isto pode-se calcular a energia de deformação que é dada por:

$$
\begin{gathered}
U=(1 / 2) \int_{V}\left[\sigma_{x} \cdot \varepsilon_{x}+\sigma_{y} \cdot \varepsilon_{y}+\sigma_{z} \cdot \varepsilon_{z}+\tau_{x y} \cdot \gamma_{x y}+\tau_{x z} \cdot \gamma_{x z}+\tau_{y z} \cdot \gamma_{y z}\right] d V \\
d V \quad \text { elemento infinitesimal de volume. }
\end{gathered}
$$


A energia potencial do elemento é definida por:

$$
\Pi I=U-\int_{s}\left[S_{x} \cdot u+S_{y} \cdot v+S_{z} \cdot W\right] d S+\Sigma P_{i} r_{i}
$$

ou,

$$
\Pi=U-\Omega
$$

Onde $S_{x}, S_{y}$ e $S_{z}$ são as componentes das forças por unidade de superfície, e $u, v$ e $w$ são os deslocamentos nas direções das respectivas forças, $P_{i}$ são as cargas concentradas na estrutura, e $r_{i}$ os deslocamentos corresppondentes às cargas $\mathrm{P}_{\mathrm{i}}$.

\subsection{O MÉTODO DOS ELEMENTOS FINITOS}

Quando se estuda um fenômeno físico de qualquer natureza, na maioria dos casos a solução desse problema recai na solução da equação diferencial que rege seu comportamento. Isso é válido para fenômenos que variam do eletromagnetismo até o escoamento de fluidos. Entretanto, a solução de uma equação diferencial existe apenas para um pequeno número de casos.

Além da dificuldade matemática já mencionada, a solução analítica, quando existir, de uma equação diferencial para uma casca é por demais trabalhosa, e inacessivel para a grande maioria dos engenheiros projetistas.

Procurando contornar essa dificuldade, o caminho adotado foi partir para a solução numérica de tais equações, cuja aceitabilidade dos resultados está na dependência direta do número de iterações, ou da discretização adotada.

Baseado nos principios do cálculo variacional, cujo rigor matemático de sua formulação não é objeto de estudo desse trabalho, o Método dos 
Elementos Finitos tem-se mostrado bastante eficiente na análise de diversos tipos de estrutura. Mormente estruturas laminares, nas quais a solução analítica, como já foi dito, é inviável.

Nesse método, considera-se a estrutura subdividida( ou discretizada) em partes, ou elementos, de dimensões finitas, para os quais se estabelecem as relaçōes entre esforços e deslocamentos. Para cada elemento, o relacionamento entre esforços e deslocamentos nodais pode ser expresso sob a seguinte forma:

$$
k_{e} \cdot u_{e}=f_{e}
$$

Onde,

$$
\begin{aligned}
& \mathbf{k}_{e} \quad \text { matriz de rigidez do elemento, } \\
& \mathbf{u}_{e} \quad \text { vetor de deslocamentos nodais, } \\
& \mathbf{f}_{e} \quad \text { vetor de esforços nodais. }
\end{aligned}
$$

De forma bastante simplista, essa relação pode ser vista como uma extensão da relação para uma mola sob ação de uma força, ou seja K. $\Delta \mathrm{x}=$ $F$, na qual a força $F$ produz um desiocamento $\Delta x$ na sua direção.

Ao estabelecer a relação entre os deslccamentos nodais de cada elemento com os deslocamentos nodais da estrutura, obter-se-á uma relação que representa o comportamento de toda estrutura. Essa relação é expressa da seguinte forma :

$$
K . U=F
$$

Onde,
K matriz de rigidez da estrutura,
U vetor de deslocamentos nodais da estrutura,
F vetor de ações atuantes na estrutura. 
Conhecendo-se a matriz de rigidez de cada elemento e as ações atuantes nos mesmos, monta-se a relação matricial anterior. A partir desta última equação, chega-se nos deslocamentos provocados pelas ações que atuam na estrutura, e finalmente nos esforços desejados. Na sequência deste trabaiho é obtida a matriz de rigidez de um elemento de membrana

\subsection{MATRIZ DE RIGIDEZ DO ELEMENTO DE MEMBRANA.}

$\mathrm{Na}$ montagem da matriz de rigidez do elemento finito de membrana, trabalha-se com coordenadas adimensionais. Esse desenvolvimento é mostrado a partir das relações que seguem.

Seja um triângulo qualquer, como mostra a figura 2.7:

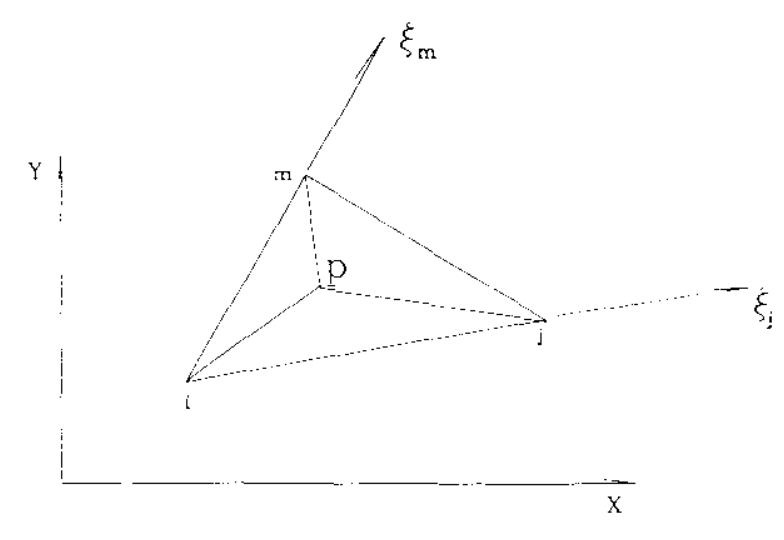

Figura 2.7 Coordenadas adimensionais.

Neste triângulo definem-se as seguintes relações : 


$$
\begin{aligned}
& \xi_{j}=\frac{\bar{\xi}_{j}}{1_{i j}} ; \quad \xi_{m}=\frac{\xi_{m}}{1_{i m}} \\
& \text { onde } \bar{\xi}_{m}=\frac{\Delta_{m}}{\Delta}
\end{aligned}
$$

$\Delta_{\mathrm{m}}$ área do triângulo $\mathrm{iPm}$

$\Delta$ área do triangulo ijm

$\mathrm{I}_{\mathrm{ij}}$ comprimento do lado ij do triângulo ijm.

Tem-se :

$$
\Delta_{\mathrm{i}}+\Delta_{\mathrm{j}}+\Delta_{\mathrm{m}}=\Delta \Rightarrow \Delta_{\mathrm{i}} / \Delta+\Delta_{\mathrm{j}} / \Delta+\Delta_{\mathrm{m}} / \Delta=1
$$

então :

$$
\xi_{i}+\xi_{j}+\xi_{m}=1
$$

Para o ponto $P(x, y)$ no interior do triângulo, verifica-se também a seguinte relação:

$$
\begin{aligned}
& x=x_{i} \xi_{i}+x_{j} \xi_{j}+x_{m} \xi_{m} \\
& y=y_{i} \xi_{i}+y_{j} \xi_{j}+y_{m} \xi_{m}
\end{aligned}
$$

Estas relações podem ser escritas na forma matricial, como segue :

$$
\begin{aligned}
& x=\varphi \cdot x^{n} \\
& y=\varphi \cdot y^{n}
\end{aligned}
$$

onde,

$$
\begin{aligned}
& \varphi=\left\{\begin{array}{lll}
\xi_{i} & \xi_{j} & \xi_{m}
\end{array}\right\} \\
& \left(x^{n}\right)^{\top}=\left\{\begin{array}{lll}
x_{i} & x_{j} & x_{m}
\end{array}\right\} \\
& \left(y^{n}\right)^{\top}=\left\{\begin{array}{lll}
y_{i} & y_{j} & y_{m}
\end{array}\right\}
\end{aligned}
$$


A partir de (2.44) e (2.45) pode-se escrever a seguinte relação matricial entre os sistemas de coordenadas adimensionais e cartesiano:

$$
\left\{\begin{array}{l}
1 \\
x \\
y
\end{array}\right\}=\left[\begin{array}{ccc}
1 & 1 & 1 \\
x_{i} & x_{j} & x_{m} \\
y_{i} & y_{j} & y_{m}
\end{array}\right]\left\{\begin{array}{l}
\xi_{i} \\
\xi_{j} \\
\xi_{m}
\end{array}\right\}
$$

ou, na forma inversa :

$$
\left\{\begin{array}{l}
\xi_{i} \\
\xi_{j} \\
\xi_{m}
\end{array}\right\}=\frac{1}{2 \Delta}\left[\begin{array}{lll}
x_{j} y_{m}-x_{m} y_{j} & y_{j}-y_{m} & x_{m}-x_{j} \\
x_{m} y_{i}-x_{i} y_{m} & y_{m}-y_{i} & x_{i}-x_{m} \\
x_{i i} y_{j}-x_{j} y_{i} & y_{i}-y_{j} & x_{j}-x_{i}
\end{array}\right]\left\{\begin{array}{l}
1 \\
x \\
y
\end{array}\right\}
$$

onde :

$$
\Delta=\frac{1}{2}\left(\mathrm{x}_{\mathrm{j}} \mathrm{y}_{\mathrm{m}}-\mathrm{x}_{\mathrm{m}} \mathrm{y}_{\mathrm{i}}+\mathrm{x}_{\mathrm{ii}} \mathrm{y}_{\mathrm{j}}-\mathrm{x}_{\mathrm{i}} \mathrm{y}_{\mathrm{m}}-\mathrm{x}_{\mathrm{m}} \mathrm{y}_{\mathrm{j}}-\mathrm{x}_{\mathrm{j}} \mathrm{y}_{\mathrm{i}}\right)
$$

Ao elemento finito indicado na figura 2.7 são agora atribuídos nós, que podem estar localizados teoricamente em qualquer ponto do elemento. A cada um destes nós são atribuídos parâmetros nodais, isto é, os deslocamentos $u_{i}$ e $\mathbf{v}_{i}$ nestes pontos.

Para cada elemento finito, adota-se uma função aproximadora para seus deslocamentos e que são expressas em função dos parâmetros nodais. No elemento finito adotado neste trabalho, supõe-se que os deslocamentos $\mathbf{u} \mathbf{e} \mathbf{v}$ variem linearmente no domínio do elemento. Com isto, as deformações $\varepsilon_{x}, \varepsilon_{y}$ e $\gamma_{x y}$ são constantes no domínio do elemento, que por isso recebe o nome de Constant Strain Triangle ( CST) . 


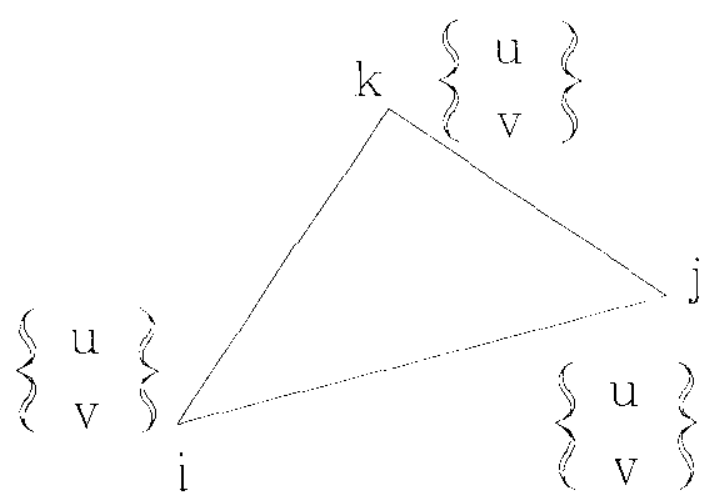

Figura 2.8 Elemento CST.

O vetor de parâmetros nodais é dado na seguinte ordem:

$$
u^{n T}=\left\{\begin{array}{llllll}
u_{1} & u_{2} & u_{3} & v_{1} & v_{2} & v_{3}
\end{array}\right\}
$$

Os deslocamentos $\mathbf{u}$ e $\mathbf{v}$ escrito em função dos deslocamentos nodais são dados por :

$$
\begin{aligned}
& u=u_{1} \cdot \xi_{1}+u_{2} \cdot \xi_{2}+u_{3} \cdot \xi_{3} \\
& v=v_{1} \cdot \xi_{1}+v_{2} \cdot \xi_{2}+v_{3} \cdot \xi_{3}
\end{aligned}
$$

Ou, na forma matricial :

$$
\mathbf{u}=\Phi \cdot \mathbf{u}^{\mathrm{n}}
$$

onde:

$$
\mathbf{u}=\left\{\begin{array}{l}
u \\
v
\end{array}\right\} \quad \Phi=\left\{\begin{array}{ll}
\varphi & 0 \\
0 & \varphi
\end{array}\right\} \quad, \quad \varphi=\left\{\begin{array}{lll}
\xi_{1} & \xi_{2} & \xi_{3}
\end{array}\right\}
$$

e,

$$
\mathbf{u}^{n}=\left\{\begin{array}{l}
* n \\
\mathbf{u} \\
v^{n}
\end{array}\right\}, \quad \vec{u}^{* n}=\left\{\begin{array}{l}
u_{1} \\
u_{2} \\
u_{3}
\end{array}\right\} \quad \text { e } \quad \vec{v}^{n}=\left\{\begin{array}{c}
v_{1} \\
v_{2} \\
v_{3}
\end{array}\right\}
$$


Com base nas considerações anteriores, passa-se ao desenvolvimento da matriz de rigidez do elemento de membrana. Voltando ao enunciado da energia potencial de um corpo tem-se:

$$
\Pi=U+\Omega
$$

Para o estado plano de tensões, a energia de deformação é dada como segue :

$$
U=(1 / 2) \int_{V}\left[\sigma_{x} \cdot \varepsilon_{x}+\sigma_{y} \cdot \varepsilon_{y}+\tau_{x y} \cdot \gamma_{x y}\right] d V
$$

ou

$$
U=(1 / 2) \int_{V} \cdot \varepsilon^{\top} \cdot \sigma d V
$$

Na relação para a energia potencial, procurar-se-á um estado no qual essa energia seja minimizada. Isso significa dizer que vale a relação :

$$
\delta \Pi=\delta(U+\Omega)=0
$$

A partir de (2.53), obtém-se o sistema final de equações lineares da membrana. A partir da equação (2.37) pode-se reescrever (2.52) como segue :

$$
U=(1 / 2) \int_{V} \varepsilon^{\top} \sigma d V=(1 / 2) \int_{V} \varepsilon^{\top} \cdot D \cdot \varepsilon d V
$$

Já foi visto que ( 2.49$)$ :

$$
\mathbf{u}=\Phi \cdot \mathbf{u}^{\mathrm{n}}
$$

Assim, de (2.38) tem-se : 


$$
\varepsilon=\text { L. } \mathbf{u}=\text { L. } \Phi \cdot \mathbf{u}^{\mathrm{n}}=\mathbf{B} \cdot \mathbf{u}^{\mathrm{n}}
$$

onde:

$B=L . \Phi$

$$
\mathbf{B}=\left|\begin{array}{cccccc}
\omega_{1} & \omega_{2} & \omega_{3} & 0 & 0 & 0 \\
0 & 0 & 0 & \eta_{1} & \eta_{2} & \eta_{3} \\
\eta_{1} & \eta_{2} & \eta_{3} & \omega_{1} & \omega_{2} & \omega_{3}
\end{array}\right|
$$

onde:

$$
\begin{aligned}
& \omega_{\mathrm{i}}=y_{j}-y_{k} \\
& \eta_{i}=x_{k}-x_{j}
\end{aligned} \quad \mathrm{ijk}=123,231,312
$$

Então, para um elemento com espessura constante, a expressão para a energia de deformação será :

$$
\begin{array}{ll}
U= & (1 / 2) \int_{A} \cdot u^{n \top} \cdot B^{\top} D^{\prime} \cdot B \cdot u^{n} d A \\
\text { com } \quad D^{\prime}=t D \\
\\
\text { té a espessura do elemento. }
\end{array}
$$

A matriz de rigidez desse elemento é dada por :

$$
K=\int_{A} \cdot B^{T} D^{\prime} \cdot B \cdot d A
$$

A partir das relações dadas em coordenadas adimensionais, desenvolve-se a matriz de rigidez . 
Ao se calcular a integral que define a matriz de rigidez do elemento, determina-se os coeficientes de rigidez desejados. Sua resolução não apresenta maiores dificuldades, e será omitida neste texto, por se julgar desnecessário. A seguir apresenta-se os coeficientes desejados de forma compacta.

$$
\mathbf{K}=\frac{\mathrm{D}}{4 \mathrm{~A}^{2}}\left[\begin{array}{ll}
\mathbf{k}_{11} & \mathbf{k}_{12} \\
\mathbf{k}_{21} & \mathbf{k}_{22}
\end{array}\right]
$$

onde

$$
\begin{aligned}
& \text { A é a área do elemento. } \\
& D=E \cdot t\left(1-v^{2}\right)
\end{aligned}
$$

Os coeficiente da matriz K são obtidos como segue :

$$
\begin{array}{lll}
\mathbf{k}_{11} & \Rightarrow & \mathbf{k}_{\mathrm{ij}}=\omega_{\mathrm{i}} \cdot \omega_{\mathrm{j}}+\mu \cdot \eta_{\mathrm{i}} \eta_{\mathrm{j}} \\
\mathbf{k}_{12} & \Rightarrow & \mathbf{k}_{\mathrm{ij}}=\nu \omega_{i} \eta_{\mathrm{j}}+\mu \omega_{j} \eta_{\mathrm{i}} \\
\mathbf{k}_{22} & \Rightarrow & \mathbf{k}_{\mathrm{ij}}=\eta_{\mathrm{i}} \eta_{\mathrm{j}}+\mu \omega_{i} \omega_{\mathrm{j}}
\end{array}
$$

onde :

$$
\begin{aligned}
& \text { para cada } i=1,3 \text { tem-se } j=1,3 \\
& \omega_{i}=y_{j}-y_{k} \\
& \eta_{i}=x_{k}-x_{j} \\
& i, j, k \text { em permutação cíclica }(123,312,231)
\end{aligned}
$$

Obtém-se dessa forma a matriz de rigidez do elemento finito de membrana. 


\section{O ELEMENTO FINITO DE PLACA.}

\subsection{INTRODUÇÃO}

$\mathrm{Na}$ formulação do elemento finito de casca plano, como já foi dito, necessita-se de um elemento finito de placa que possua formulação compatível com o elemento finito de membrana. Neste capítulo apresentase o elemento de placa usado nesse trabalho. Pertencendo à classe dos elementos com nove graus de liberdade, o elemento DKT ( assim chamado por se aplicar a teoria de Kirchhoff nos pontos nodais), segundo BATOZ (1980), tem-se mostrado eficiente no estudo do comportamento de placas. Procura-se mostrar detalhadamente todo procedimento usado na montagem da matriz de rigidez desse elemento.

Antes, porém, será obordado o comportamento de uma placa sob efeito de cargas perpendiculares ao seu plano médio. Nesse estudo que se faz sobre placas, considera-se primeiro o comportamento das placas sob influência do esforço cortante ( placa espessa ). Em seguida, despreza-se a influência desse esforço, para se estudar apenas placas delgadas.

\subsection{HIPÓTESES BÁSICAS DA PLACA SOLICITADA À FLEXÃO.}

Seja uma placa de contorno qualquer, submetida a um carregamento 
$p(x, y)$ qualquer. Um elemento genérico $d x . d y . h$ da placa estará sujeito às tensões $\sigma_{x}, \sigma_{y}, \sigma_{z}, \tau_{x y}, \tau_{x z}, \tau_{y x}, \tau_{y z}, \tau_{z x}, \tau_{z y}$. Sabe-se que $\tau_{z y}=\tau_{y z}, \tau_{x z}=\tau_{z x}, \tau_{z y}=\tau_{y z}$. Assim, as tensões atuantes nas faces do elemento estão indicadas na figura 3.1:
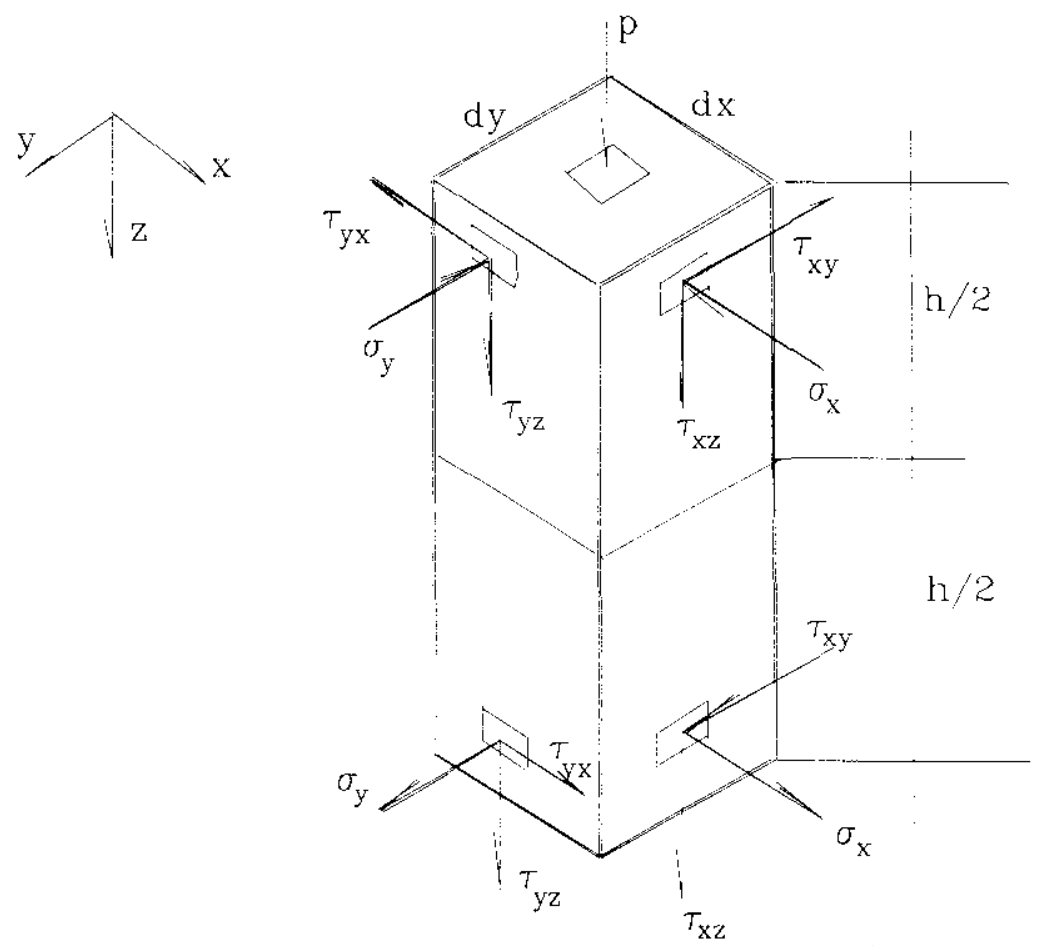

(a)
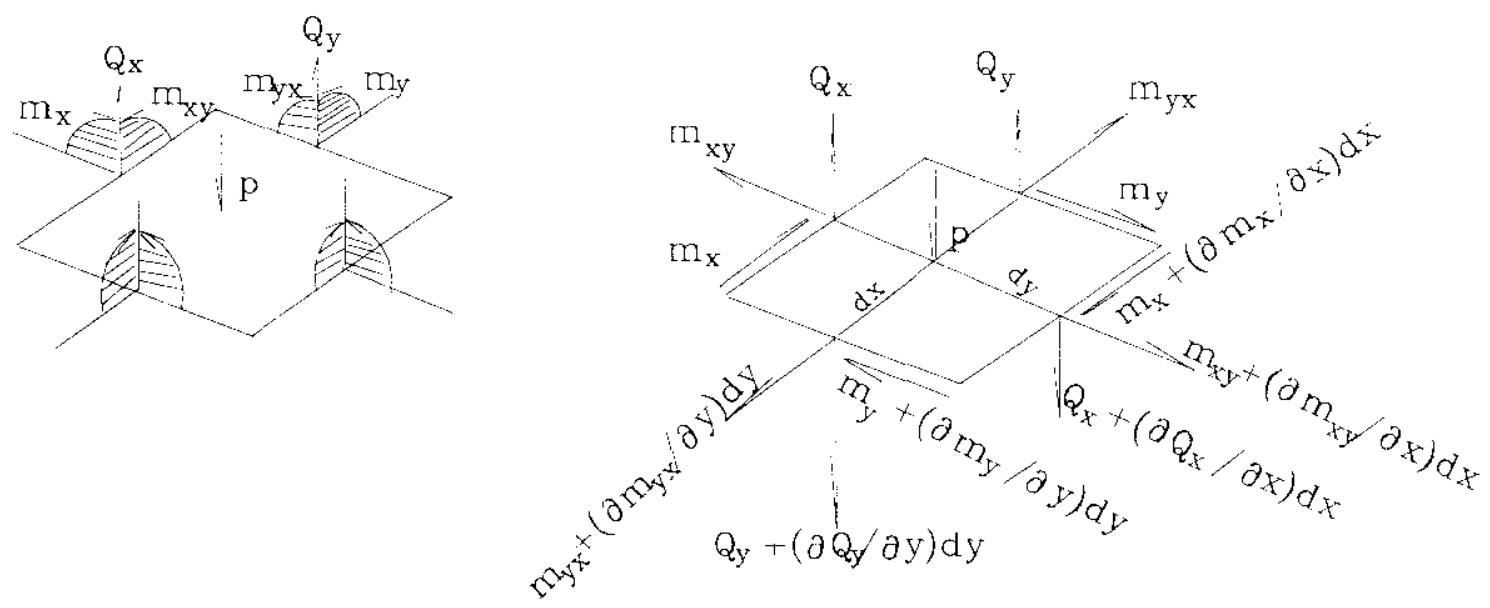

(b)

(C)

Figura 3.1 Placa sob carregamento. 
A orientação dos esforços é dada conforme a seguinte convenção :

a. As tensões $\sigma$ são positivas quando provocam tração na face do elemento; as tensões $\tau$ são positivas se coincidem com o sentido positivo dos eixos.

b. Os momentos fletores são positivos se provocam tração na fibra inferior;

c. Os momentos volventes são positivos quando seu vetor é emergente da face considerada.;

d. As forças cortantes são positivas se, olhando o eixo crescente da esquerda para a direita, tendem a girar o elemento no sentido horário.

Pode-se definir então, os esforços solicitantes que atuam nas faces do elemento:

$$
\begin{array}{lr}
\mathrm{m}_{\mathrm{x}}=\int \sigma_{\mathrm{x}} \mathrm{zdz} & (-\mathrm{h} / 2 \leq \mathrm{z} \leq \mathrm{h} / 2) \\
\mathrm{m}_{\mathrm{y}}=\int \sigma_{\mathrm{y}} \mathrm{zdz} & (-\mathrm{h} / 2 \leq \mathrm{z} \leq \mathrm{h} / 2) \\
\mathrm{m}_{\mathrm{yx}}=\int \tau_{\mathrm{yx}} \mathrm{zdz}=\int \tau_{\mathrm{xy}} \mathrm{zdz}=-\mathrm{m}_{\mathrm{xy}} & (-\mathrm{h} / 2 \leq \mathrm{z} \leq \mathrm{h} / 2) \\
\mathrm{q}_{\mathrm{x}}=\int \tau_{\mathrm{xz}} \mathrm{dz} & (-\mathrm{h} / 2 \leq \mathrm{z} \leq \mathrm{h} / 2) \\
\mathrm{q}_{\mathrm{y}}=\int \tau_{\mathrm{yz}} \mathrm{dz} & (-\mathrm{h} / 2 \leq \mathrm{z} \leq \mathrm{h} / 2)
\end{array}
$$

Na teoria de placas admite-se válidas as seguintes hipóteses :

a. O material é elástico-linear e segue a lei de Hooke;

b Os deslocamentos verticais $\mathbf{w}$ são pequenos comparados com a espessura $\mathbf{h}$ da placa;

c. Os deslocamentos horizontais dos pontos do plano médio são despreziveis. 


\subsection{CONDIÇÕES DE EQUILÍBRIO DE UM ELEMENTO DA PLACA.}

Considerando-se positivos os sentidos dos esforços indicados na figura 3.1, pode-se escrever as seguintes equações de equilibrio :

de forças verticais,

$$
\left(\left(\frac{\partial q_{x}}{\partial x}\right) d x\right) d y+\left(\left(\frac{\partial q_{y}}{\partial y}\right) d y\right) d x+p d x d y=0
$$

ou :

$$
\frac{\partial q_{x}}{\partial x}+\frac{\partial q_{y}}{\partial y}=-p
$$

equilibrio do momento em torno do eixo $x$,

$$
\left(q_{y} d x\right) d y-\left(\left(\frac{\partial m_{y}}{\partial y}\right) d y\right) d x+\left(\left(\frac{\partial m_{x y}}{\partial x}\right) d x\right) d y=0
$$

ou,

$$
\frac{\partial \mathrm{m}_{\mathrm{y}}}{\partial \mathrm{y}}-\frac{\partial \mathrm{m}_{\mathrm{xy}}}{\partial \mathrm{x}}=\mathrm{q}_{\mathrm{y}}
$$

equilibrio do momento em torno do eixo y,

$$
\left(q_{x} d y\right) d x-\left(\left(\frac{\partial m_{x}}{\partial x}\right) d x\right) d y+\left(\left(\frac{\partial m_{y x}}{\partial y}\right) d y\right) d x=0
$$

ou,

$$
\frac{\partial \mathbf{m}_{x}}{\partial \mathbf{x}}-\frac{\partial \mathrm{m}_{\mathrm{xy}}}{\partial \mathrm{y}}=\mathrm{q}_{\mathrm{x}}
$$


Pode-se agrupar as tres equações como segue :

$$
\frac{\partial^{2} \mathrm{~m}_{\mathrm{x}}}{\partial \mathrm{x}^{2}}-2 \frac{\partial^{2} \mathrm{~m}_{\mathrm{xy}}}{\partial \mathrm{x} \partial \mathrm{y}}+\frac{\partial^{2} \mathrm{~m}_{\mathrm{y}}}{\partial \mathrm{y}^{2}}=-\mathrm{p}
$$

As equações de equilíbrio nas tensões são $(2.19,2.20$ e 2.21) :

$$
\begin{aligned}
& \frac{\partial \sigma_{x}}{\partial x}+\frac{\partial \tau_{x y}}{\partial y}+\frac{\partial \tau_{x z}}{\partial z}+X=0 \\
& \frac{\partial \tau_{x y}}{\partial x^{*}}+\frac{\partial \sigma_{y}}{\partial y}+\frac{\partial \tau_{y z}}{\partial z}+Y=0 \\
& \frac{\partial \tau_{x z}}{\partial x}+\frac{\partial \tau_{y z}}{\partial y}+\frac{\partial \sigma_{z}}{\partial z}+Z=0
\end{aligned}
$$

Também, tem-se como válidas as relações de Hooke (2.34) :

$$
\begin{aligned}
& \varepsilon_{x}=\frac{1}{E}\left[\sigma_{x}-v\left(\sigma_{y}+\sigma_{z}\right)\right]=\frac{\partial u}{\partial x} \\
& \varepsilon_{y}=\frac{1}{E}\left[\sigma_{y}-v\left(\sigma_{x}+\sigma_{z}\right)\right]=\frac{\partial v}{\partial y} \\
& \varepsilon_{z}=\frac{1}{E}\left[\sigma_{z}-v\left(\sigma_{x}+\sigma_{y}\right)\right]=\frac{\partial w}{\partial z} \\
& \gamma_{x y}=\frac{1}{G} \tau_{x y}=\frac{\partial v}{\partial x}+\frac{\partial u}{\partial y} \\
& \gamma_{x z}=\frac{1}{G} \tau_{x z}=\frac{\partial u}{\partial z}+\frac{\partial w}{\partial x} \\
& \gamma_{y z}=\frac{1}{G} \tau_{y z}=\frac{\partial v}{\partial z}+\frac{\partial w}{\partial y}
\end{aligned}
$$




\subsection{DETERMINAÇÃO DAS TENSÕES PARA PLACAS COM EFEITO DO ESFORÇO CORTANTE}

Supōe-se que as tensões $\sigma_{x}, \sigma_{y}$ e $\tau_{x y}$ variem linearmente. Assim partindo -se de uma faixa de largura unitária na direção $x$, pode-se escrever:

$$
\sigma_{x}=\frac{M_{x}}{I_{z}} z
$$

então ,

$$
\sigma_{\mathrm{x}}=\frac{12 \cdot \mathrm{M}_{\mathrm{x}}}{\mathrm{h}^{3}} \mathrm{z} \quad \text { para } \quad \mathrm{I}_{\mathrm{z}}=4 \frac{\mathrm{h}^{3}}{12}
$$

Analogamente

$$
\sigma_{y}=\frac{12 \cdot M_{y}}{h^{3}} z \quad \text { e, } \quad \tau_{x y}=\frac{12 \cdot M_{x y}}{h^{3}} z
$$

A partir da figura 3.1 tem-se as seguintes condiçōes de contorno:

$$
\begin{array}{ll}
\sigma_{z}=-p(x, y) & \text { para } z=-h / 2 \\
\sigma_{z}=0 & \text { para } z=+h / 2 \\
\tau_{x z}=\tau_{y z}=0 & \text { para } z=+/-h / 2
\end{array}
$$


Substituindo-se as tensões (3.8) nas duas últimas equações de equilibrio (3.7), desprezadas as forças de massa, e integrando-se ao longo de z, com o auxilio da última condição de contorno (3.9), obtêm-se :

$$
\begin{aligned}
& \tau_{x z}=\frac{3 Q_{x}}{2 h}\left[1-\left(\frac{2 z}{h}\right)^{2}\right] \\
& e \\
& \tau_{y z}=\frac{3 Q_{y}}{2 h}\left[1-\left(\frac{2 z}{h}\right)^{2}\right]
\end{aligned}
$$

Substituindo as tensões dadas em (3.10) na primeira das equações de equilibrio (3.7), utilizando-se da (3.2) e integrando-se ao longo de $z$, com auxilio das duas primeiras condições de contorno (3.9), obtém-se :

$$
\sigma_{z}=-\frac{3 p}{4}\left[\frac{2}{3}-\frac{2 z}{h}+\frac{1}{3}\left(\frac{2 z}{h}\right)^{2}\right]
$$

Na teoria de placas espessas (REISSNER, 1945) , as seções planas não permanecem planas após as deformaçōes. Assim, com o objetivo de simplificar 0 tratamento matemático do problema, calculam-se 0 deslocamento médio $w_{o}$ e os giros médios da seção $\theta_{x}$ e $\theta_{y}$, e supõe-se que, com estes valores, as seções permaneçam pianas mas não normais à superficie média deformada.

Estes valores são calculados igualando-se o trabalho realizado para executar os giros médios, e os deslocamentos médios, ao trabalho das tensões correspondentes ao efetuar os deslocamentos reais $u, v e w$, ou seja: 


$$
\begin{array}{lc}
\int_{-\mathrm{h} / 2}^{+\mathrm{h} / 2} \sigma_{\mathrm{x}} \mathrm{udz}=\mathrm{M}_{\mathrm{x}} \theta_{\mathrm{x}} \quad ; \quad \int_{-\mathrm{h} / 2}^{\mathrm{hh} / 2} \tau_{\mathrm{xy}} \mathrm{vdz}=\mathrm{M}_{\mathrm{xy}} \theta_{\mathrm{y}} \\
\int_{-\mathrm{h} / 2}^{+\mathrm{h} / 2} \sigma_{\mathrm{y}} \mathrm{vdz}=\mathrm{M}_{\mathrm{y}} \theta_{\mathrm{y}} \quad ; \quad \int_{-\mathrm{h} / 2}^{+\mathrm{h} / 2} \tau_{\mathrm{xy}} \mathrm{udz}=\mathrm{M}_{\mathrm{xy}} \theta_{\mathrm{x}} \\
\int_{-\mathrm{h} / 2}^{+\mathrm{h} / 2} \tau_{\mathrm{xz}} \mathrm{wdz}=\mathrm{Q}_{\mathrm{x}} \mathrm{W}_{\mathrm{o}} \quad ; \quad & \int_{-\mathrm{h} / 2}^{+\mathrm{h} / 2} \tau_{\mathrm{yz}} \mathrm{Wdz}=\mathrm{Q}_{\mathrm{y}} \mathrm{W}_{\mathrm{o}}
\end{array}
$$

Utilizando-se das equações (3.8) e (3.10), e substituindo-as em (3.12) obtém-se :

$$
\begin{aligned}
& \theta_{\mathrm{x}}=\frac{12}{\mathrm{~h}^{3}} \int_{-\mathrm{h} / 2}^{+\mathrm{h} / 2} \mathrm{uzd} \mathrm{z} \\
& \theta_{\mathrm{y}}=\frac{12}{\mathrm{~h}^{3}} \int_{-\mathrm{h} / 2}^{+\mathrm{h} / 2} \mathrm{vzdz} \\
& \mathrm{w}_{\mathrm{o}}=\frac{3}{2 \mathrm{~h}} \int_{-\mathrm{h} / 2}^{+\mathrm{h} / 2} \mathrm{w}\left[1-\left(\frac{2 \mathrm{z}}{\mathrm{h}}\right)^{2}\right] \mathrm{dz}
\end{aligned}
$$

Supondo-se que as seções permaneçam planas após as deformações, como já foi citado anteriormente, tem-se que os valores dos deslocamentos u e v são os seguintes:

$$
\mathrm{u}=-\mathrm{z} \cdot \theta_{\mathrm{x}} \quad \mathrm{v}=-\mathrm{z} \cdot \theta_{\mathrm{y}}
$$

Substituindo-se as tensões (3.10) nas duas últimas relações dadas segundo a lei Hooke (3.7), e integrando-se em relação a $z$ de $-h / 2$ a $+h / 2$, sabendo-se que $\partial u / \partial z=-\theta_{x}$ e $\partial v / \partial z=-\theta_{y}$ (3.14), obtêm-se: 


$$
\begin{aligned}
& \frac{\partial \mathrm{u}}{\partial \mathrm{z}}+\frac{\partial \mathrm{w}}{\partial \mathrm{x}}=\frac{6}{5} \frac{2(1+v)}{\mathrm{Eh}} \mathrm{Q}_{\mathrm{x}} \\
& \frac{\partial \mathrm{v}}{\partial \mathrm{z}}+\frac{\partial \mathrm{w}}{\partial \mathrm{y}}=\frac{6}{5} \frac{2(1+v)}{\mathrm{Eh}} \mathrm{Q}_{\mathrm{y}}
\end{aligned}
$$

ou,

$$
\begin{aligned}
& \theta_{x}=\frac{\partial w}{\partial x}-\gamma_{x} \\
& \theta_{y}=\frac{\partial w}{\partial y}-\gamma_{y}
\end{aligned}
$$

onde,

$$
\begin{aligned}
\bar{\gamma}_{\mathrm{x}} & =\frac{6}{5} \frac{2(1+v)}{\mathrm{Eh}} \mathrm{Q}_{\mathrm{x}} \\
\mathrm{e} & \\
\bar{\gamma}_{\mathrm{y}} & =\frac{6}{5} \frac{2(1+v)}{\mathrm{Eh}} \mathrm{Q}_{\mathrm{y}}
\end{aligned}
$$

Definindo-se :

$$
\beta_{x}=-\theta_{x}
$$

e,

$$
\beta_{y}=-\theta_{y}
$$

pode-se reescrever as relações anteriores como se segue :

$$
\begin{aligned}
& \beta_{\mathrm{x}}=-\frac{\partial \mathrm{w}}{\partial \mathrm{x}}+\bar{\gamma}_{\mathrm{x}}=-\mathrm{w}_{, \mathrm{x}}+\bar{\gamma}_{\mathrm{x}} \\
& \mathrm{e}, \\
& \beta_{\mathrm{y}}=-\frac{\partial \mathrm{w}}{\partial \mathrm{y}}+\bar{\gamma}_{\mathrm{y}}=-\mathrm{w}_{, \mathrm{y}}+\bar{\gamma}_{\mathrm{y}} \\
& \text { onde : } \frac{\partial \mathrm{w}}{\partial \mathrm{x}}=\mathrm{w}_{, \mathrm{x}} \text { e } \frac{\partial \mathrm{w}}{\partial \mathrm{y}}=\mathrm{w}_{, y}
\end{aligned}
$$


ou,

$$
\begin{gathered}
\bar{\gamma}_{\mathrm{x}}=\mathrm{w}_{, \mathrm{x}}+\beta_{\mathrm{x}} \\
- \\
\bar{\gamma}_{\mathrm{y}}=\mathrm{w}_{, y}+\beta_{\mathrm{y}}
\end{gathered}
$$

Assim, os deslocamentos podem ser expressos como segue:

$$
\begin{aligned}
& u=-z \theta_{x}=z \beta_{x} \\
& v=-z \theta_{y}=z \beta_{y}
\end{aligned}
$$

Substituindo-se (3.21) nas relações expressas na lei de Hooke, obtém-se as seguintes expressões para as deformações :

$$
\begin{aligned}
& \varepsilon_{\mathrm{x}}=\mathrm{z} \frac{\partial \beta_{\mathrm{x}}}{\partial \mathrm{x}}=\mathrm{z} \beta_{\mathrm{x}, \mathrm{x}} \\
& \varepsilon_{\mathrm{y}}=\mathrm{z} \frac{\partial \beta_{\mathrm{y}}}{\partial \mathrm{y}}=\mathrm{z} \beta_{\mathrm{y}, \mathrm{y}} \\
& \gamma_{\mathrm{xy}}=\mathrm{z}\left(\frac{\partial \beta_{\mathrm{x}}}{\partial \mathrm{y}}+\frac{\partial \beta_{\mathrm{y}}}{\partial \mathrm{x}}\right)=\mathrm{z}\left(\beta_{\mathrm{x}, \mathrm{y}}+\beta_{\mathrm{y}, \mathrm{x}}\right) \\
& \gamma_{\mathrm{xz}}=\gamma_{\mathrm{x}}=\beta_{\mathrm{x}}+\mathrm{w}_{, \mathrm{x}} \\
& \gamma_{\mathrm{yz}}=\gamma_{\mathrm{y}}=\beta_{\mathrm{y}}+\mathrm{w}_{, y}
\end{aligned}
$$

Que podem ser expressas da seguinte forma matricial :

$$
\varepsilon=\mathrm{Z \kappa}
$$


onde :

$$
\varepsilon=\left\{\begin{array}{c}
\varepsilon_{x} \\
\varepsilon_{y} \\
\gamma_{x y}
\end{array}\right\} \quad \text { e } \quad \kappa=\left\{\begin{array}{c}
\beta_{x, x} \\
\beta_{y, y} \\
\beta_{x, y}+\beta_{y, x}
\end{array}\right\}
$$

e

$$
\gamma=\left\{\begin{array}{l}
\gamma_{x z} \\
\gamma_{y z}
\end{array}\right\}=\left\{\begin{array}{c}
- \\
\gamma_{x} \\
- \\
\gamma_{y}
\end{array}\right\}=\left\{\begin{array}{l}
\beta_{x}+w_{, x} \\
\beta_{y}+w_{, y}
\end{array}\right\}
$$

Reescrevendo-se as relações de Hooke, de forma que as tensões estjam em função das deformações (lembrando que $\varepsilon_{z}$, é considerada desprezivel ) resulta em :

$$
\sigma_{b}=\mathbf{z} \mathbf{D}_{\mathbf{b}} \kappa+\sigma_{z} \cdot \mathbf{D}_{s}
$$

onde:

$\kappa$ é o vetor de curvaturas dado em (3.23),

$$
\begin{aligned}
& \sigma_{\mathrm{b}}=\left\{\begin{array}{l}
\sigma_{\mathrm{x}} \\
\sigma_{\mathrm{y}} \\
\tau_{\mathrm{xy}}
\end{array}\right\}, \quad \mathbf{D}_{\mathrm{b}}=\frac{\mathrm{E}^{2}}{1-v^{2}}\left[\begin{array}{ccc}
1 & v & 0 \\
v & 1 & 0 \\
0 & 0 & \frac{1-v}{2}
\end{array}\right], \\
& \sigma_{\mathrm{z}}=\frac{-3 \mathrm{p} v}{4(1-v)}\left(\frac{3}{2}-\frac{2 \mathrm{z}}{\mathrm{h}}+\frac{1}{3}\left(\frac{2 \mathrm{z}}{\mathrm{h}}\right)^{3}\right) \quad \mathrm{e} \quad \mathbf{D}_{\mathrm{s}}=\frac{v}{1-v}\left\{\begin{array}{l}
1 \\
1 \\
0
\end{array}\right\}
\end{aligned}
$$

e,

$$
\sigma_{s}=\left\{\begin{array}{c}
\tau_{x z} \\
\tau_{y z}
\end{array}\right\}=\mathbf{G}\left\{\begin{array}{c}
- \\
\gamma_{x} \\
- \\
\gamma_{y}
\end{array}\right\}
$$




\subsection{DETERMINAÇÃO DOS ESFORÇOS}

Para se obter os esforços em função das deformações, basta substituir as relações dadas em (3.24), nas relações (3.6), e integrar no domínio da placa.

Assim, para os momentos vem :

$M=D \cdot k+M_{0}$

onde:

k é o vetor de curvaturas dado em (3.23), e

$$
=\left\{\begin{array}{l}
M_{x} \\
M_{y} \\
M_{x y}
\end{array}\right\}, \quad D=\frac{E^{3} h^{3}}{12\left(1-v^{2}\right)}\left[\begin{array}{ccc}
1 & v & 0 \\
v & 1 & 0 \\
0 & 0 & \frac{1-v}{2}
\end{array}\right] \quad, \quad M_{o}=\frac{p^{2}}{10}\left(\frac{v}{1-v}\right)\left\{\begin{array}{l}
1 \\
1 \\
0
\end{array}\right\}
$$

E para o esforço cortante vem :

$$
\mathbf{Q}=\mathbf{D}_{\mathrm{q}} \gamma
$$

onde :

$$
\mathrm{Q}=\left\{\begin{array}{l}
\mathrm{Q}_{\mathrm{x}} \\
\mathrm{Q}_{\mathrm{y}}
\end{array}\right\}, \quad \mathrm{D}_{\mathrm{q}}=\frac{6}{5} \mathrm{Gh}\left[\begin{array}{ll}
1 & 0 \\
0 & 1
\end{array}\right] \quad \text { e } \quad \gamma=\left\{\begin{array}{l}
\gamma_{\mathrm{xz}} \\
\gamma_{\mathrm{yz}}
\end{array}\right\}
$$




\subsection{EQUAÇÃO DIFERENCIAL DAS PLACAS.}

Com as equações dadas no item anterior, pode-se obter os esforços que solicitam a placa num ponto qualquer $\left(m_{x}, m_{y}, m_{x y}, q_{x}, q_{y}\right)$, se se conhece a função que define a elástica na placa $w(x, y)$. Substituindo-se os momentos (3.25) na equação das placas (3.5) tem-se :

$$
\nabla^{4} w=\frac{1}{D}\left(p-\frac{h^{2}}{10} \frac{2-v}{1-v} \nabla^{2} p\right)
$$

onde :

$$
\nabla^{4}=\nabla^{2} \nabla^{2} \quad \text { e } \quad \nabla^{2}=\left(\frac{\partial^{2}}{\partial \mathrm{x}^{2}}+\frac{\partial^{2}}{\partial \mathrm{y}^{2}}\right) .
$$

\subsection{ENERGIA DE DEFORMAÇÃO DE PLACAS COM EFEITO DO ESFORÇO CORTANTE}

A energia de deformação compõe-se das parcelas devidas à flexão $\left(U_{b}\right)$ e ao cisalhamento $\left(U_{s}\right)$. Assim :

$$
\mathrm{U}=\mathrm{U}_{\mathrm{b}}+\mathrm{U}_{\mathrm{s}}
$$

onde :

$$
U_{b}=(1 / 2) \int_{A} \kappa^{\top} \cdot D_{b} \cdot \kappa d x d y
$$

e,

$$
\mathrm{U}_{\mathrm{s}}=(1 / 2) \int_{\mathrm{A}} \gamma^{\top} \cdot \mathrm{D}_{\mathrm{s}} \cdot \gamma d x d y
$$


Pode-se então escrever explicitamente as relações para as energias de deformação :

$$
\begin{array}{r}
U_{b}=\left(E \cdot h^{3} /\left(24\left(1-v^{2}\right)\right)\right) \cdot \int_{A}\left\{\beta_{x, x}^{2}+\beta_{y, y}^{2}+2 v \beta_{y, y} \beta_{x, x}+\right. \\
\left.((1-v) / 2) \cdot\left(\beta_{y, x}+\beta_{x, y}\right)^{2}\right\} d x d y
\end{array}
$$

e,

$$
U_{s}=\left(\operatorname{Ehr} /(4(1+v)) \int_{A}\left\{\left(w_{, x}+\beta_{x}\right)^{2}+\left(w_{, y}+\beta_{, y}\right)^{2}\right\} d x d y\right.
$$

\subsection{MONTAGEM DA MATRIZ DE RIGIDEZ DO ELEMENTO DKT.}

A partir da expressão (3.28) pode-se obter um elemento finito com efeito do esforço cortante. Entretanto, esșe trabalho tem como objetivo o estudo de placas delgadas. Em tais placas, o efeito do esforço cortante é desprezivel na presença da parcela devida à flexão.Isso significa dizer que a expressão (3.28) pode ser aproximada por :

$$
U=U_{b}
$$

A partir da relação anterior, pode-se desenvolver a matriz de rigidez de um elemento finito para placas delgadas.

Baseado na hipótese de Kirchhoff para placas delgadas( "pontos da placa originalmente normais à superfície média indeformada, permanecem normais à superfície média deformada"), o elemento usado neste trabalho, chamado de DKT( Discrete Kirchhoff Teory), tem-se mostrado 
numericamente preciso na análise de placas delgadas. Esse elemento possui 9 graus de liberdade (deslocamento vertical w e as rotações $\theta_{x}$ e $\theta_{y}$ nos vértices), como pode ser visto na figura 3.2. O seu vetor de parâmetros nodais é dado como segue :

$$
\mathbf{u}^{\top}=\left[\begin{array}{lllllllll}
w_{1} & \theta_{x 1} & \theta_{y 1} & w_{2} & \theta_{x 2} & \theta_{y 2} & w_{3} & \theta_{x 3} & \theta_{y 3}
\end{array}\right]
$$

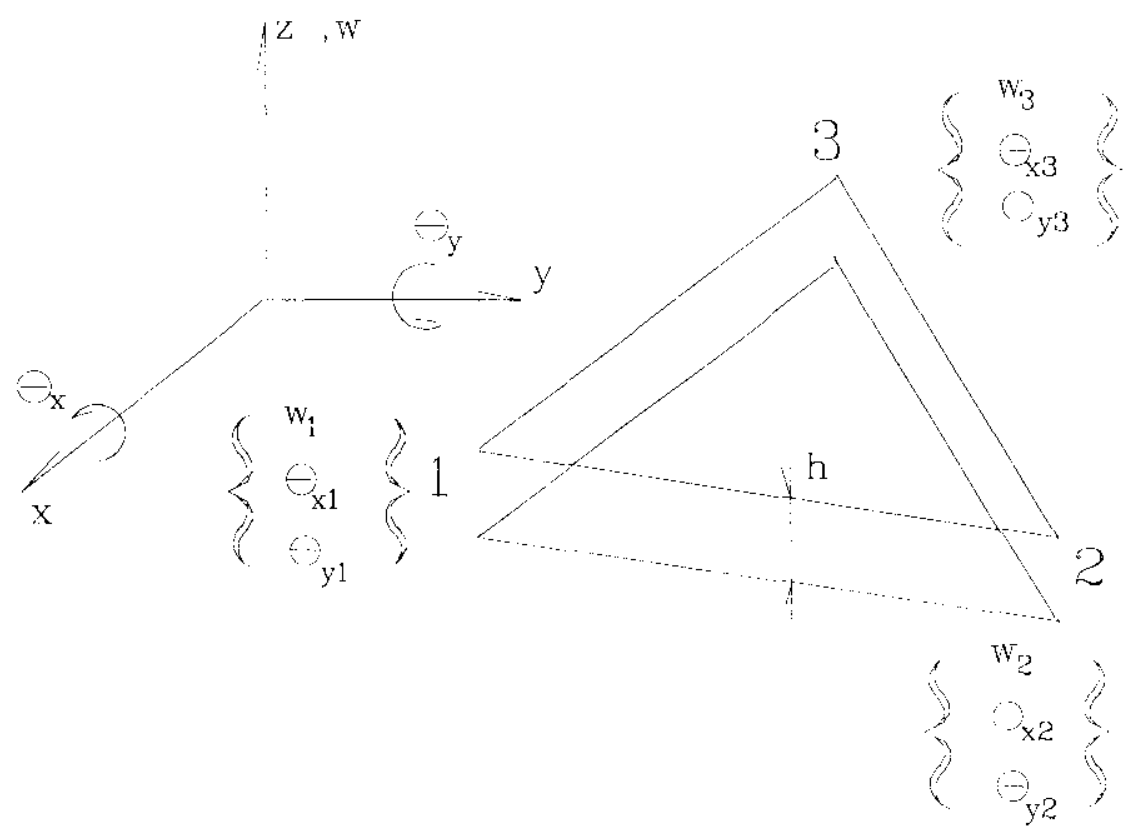

Figura 3.2 Elemento DKT.

Neste elemento admite-se que $\beta_{x}$ e $\beta_{y}$ variem quadraticamente sobre o mesmo. Assim, seja a função aproximadora para $\beta_{x}$ : 


$$
\beta_{x}=\Phi . \alpha
$$

sendo

$$
\Phi=\left[\begin{array}{llllll}
1 & \xi & \eta & \xi^{2} & \xi \eta & \eta^{2}
\end{array}\right] \quad \text { e } \quad \alpha=\left\{\begin{array}{l}
\alpha_{1} \\
\alpha_{2} \\
\alpha_{3} \\
\alpha_{4} \\
\alpha_{5} \\
\alpha_{6}
\end{array}\right\}
$$

onde :

$$
\begin{aligned}
& \alpha_{i} \quad \text { constantes a serem determinadas, } \\
& \xi \text { e } \eta \text { são as coordenadas de área. }
\end{aligned}
$$

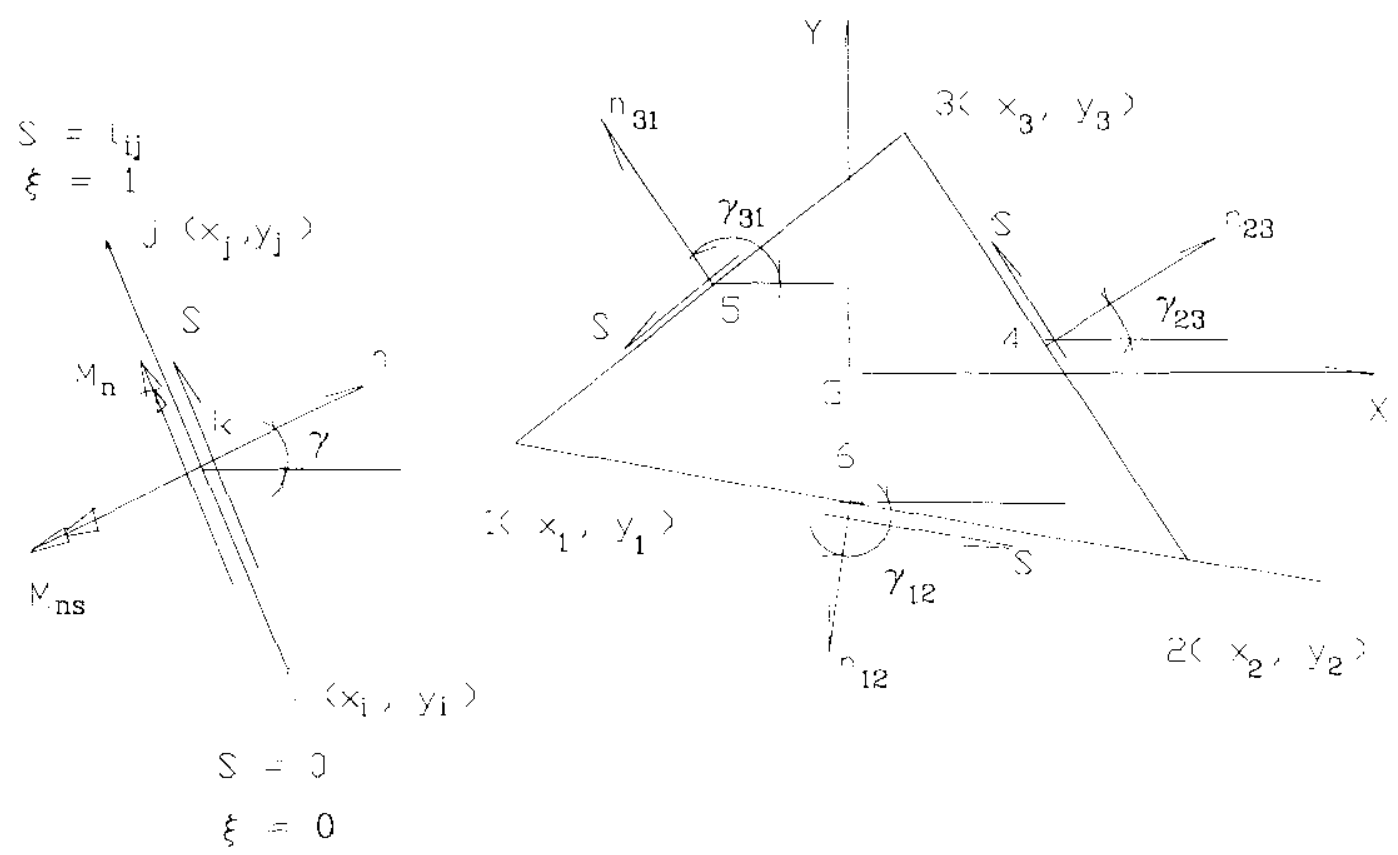

Figura 3.3 Relações geométricas no elemento triangular. 
Onde :

$$
\begin{aligned}
& \mathrm{I}_{\mathrm{ij}}=\left(\mathrm{x}_{\mathrm{ij}}^{2}+\mathrm{y}_{\mathrm{ij}}^{2}\right)^{1 / 2} ; \mathrm{x}_{\mathrm{ij}}=\mathrm{x}_{\mathrm{j}}-\mathrm{x}_{\mathrm{i}} ; \mathrm{y}_{\mathrm{ij}}=\mathrm{y}_{\mathrm{j}}-\mathrm{y}_{\mathrm{i}} \\
& \gamma_{i j}=\left(\overrightarrow{\mathrm{x}}, \overrightarrow{\mathrm{n}_{\mathrm{ij}}}\right) \gamma_{i j}=\left(\overrightarrow{\mathrm{x}}, \overrightarrow{\gamma_{\mathrm{ij}}}\right) ; \xi=\mathrm{S} / \mathrm{I}_{\mathrm{ij}} ; \\
& \mathrm{x}=\mathrm{x}_{\mathrm{i}}-\xi \mathrm{x}_{\mathrm{ij}} ; \mathrm{y}=\mathrm{y}_{\mathrm{i}}-\xi \mathrm{y}_{\mathrm{ij}} \\
& \mathrm{C}=\operatorname{Cos} \gamma_{\mathrm{ij}}=-\mathrm{y}_{\mathrm{ij}} / \mathrm{I}_{\mathrm{ij}} ; \mathrm{S}=\operatorname{Sin} \gamma_{\mathrm{ij}}=+\mathrm{x}_{\mathrm{ij}} / \mathrm{I}_{\mathrm{ij}} \\
& \mathrm{x}_{\mathrm{k}}=(1 / 2)\left(\mathrm{x}_{\mathrm{i}}+\mathrm{x}_{\mathrm{j}}\right) ; \mathrm{y}_{\mathrm{k}}=(1 / 2)\left(\mathrm{y}_{\mathrm{i}}+\mathrm{y}_{\mathrm{j}}\right)
\end{aligned}
$$

Aplicando-se a função para $\beta_{x}$ nos pontos de 1 a 6 , conforme figura 3.3 , terse-á

$$
\left\{\begin{array}{l}
\beta_{x 1} \\
\beta_{x 2} \\
\beta_{x 3} \\
\beta_{x 4} \\
\beta_{x 5} \\
\beta_{x 6}
\end{array}\right\}=\left[\begin{array}{cccccc}
1 & 0 & 0 & 0 & 0 & 0 \\
1 & 1 & 0 & 1 & 0 & 0 \\
1 & 0 & 1 & 0 & 0 & 1 \\
1 & 1 / 2 & 1 / 2 & 1 / 4 & 1 / 4 & 1 / 4 \\
1 & 0 & 1 / 2 & 0 & 0 & 1 / 4 \\
1 & 1 / 2 & 0 & 1 / 4 & 0 & 0
\end{array}\right] \cdot\left\{\begin{array}{l}
\alpha_{1} \\
\alpha_{2} \\
\alpha_{3} \\
\alpha_{4} \\
\alpha_{5} \\
\alpha_{6}
\end{array}\right\}
$$

A partir da equação anterior obtém-se :

$$
\left\{\begin{array}{l}
\alpha_{1} \\
\alpha_{2} \\
\alpha_{3} \\
\alpha_{4} \\
\alpha_{5} \\
\alpha_{6}
\end{array}\right\}=\left[\begin{array}{cccccc}
1 & 0 & 0 & 0 & 0 & 0 \\
-3 & -1 & 0 & 0 & 0 & 4 \\
-3 & 0 & -1 & 0 & 4 & 0 \\
2 & 2 & 0 & 0 & 0 & -4 \\
4 & 0 & 0 & 4 & -4 & -4 \\
2 & 0 & 2 & 0 & -4 & 0
\end{array}\right]\left\{\begin{array}{l}
\beta_{x 1} \\
\beta_{x 2} \\
\beta_{x 3} \\
\beta_{x 4} \\
\beta_{x 5} \\
\beta_{x 6}
\end{array}\right\}
$$

A partir das relações anteriores (3.34) e (3.37) obtém-se a expressão para $\beta_{\mathrm{x}}$. De modo análogo se obtém também $\beta_{\mathrm{y}}$. A expressões são dadas por : 


$$
\beta_{x}=\sum_{i=1}^{6} \mathrm{~N}_{\mathrm{i}} \beta_{x i} \quad ; \quad \beta_{y}=\sum_{i=1}^{6} \mathrm{~N}_{\mathrm{i}} \beta_{y i}
$$

onde $\beta_{x i}$ e $\beta_{y i}$ são os valores nodais nos vértices e nos pontos médios dos lados, conforme figura $3.3 ; N_{i}(\xi, \eta)$ são as funções de forma dadas como segue :

$$
\begin{aligned}
& \mathrm{N}_{1}=2(1-\xi-\eta)(1 / 2-\xi-\eta) \\
& \mathrm{N}_{2}=\xi(2 \xi-1) \\
& \mathrm{N}_{3}=\eta(2 \eta-1) \\
& \mathrm{N}_{4}=4 \xi \eta \\
& \mathrm{N}_{5}=4 \eta(1-\xi-\eta) \\
& \mathrm{N}_{6}=4 \xi(1-\xi-\eta)
\end{aligned}
$$

Admite-se também que a função que descreve o comportamento do deslocamento vertical ( $w$ ), ao longo dos lados, é cúbica. Então seja a seguinte relação :

$$
w(s)=\Phi \cdot \alpha
$$

onde,

$$
\begin{aligned}
& \Phi=\left\{\begin{array}{llll}
1 & \mathrm{~s} & \mathrm{~s}^{2} & \mathrm{~s}^{3}
\end{array}\right\} \\
& \alpha^{\top}=\left\{\begin{array}{llll}
\alpha_{0} & \alpha_{1} & \alpha_{2} & \alpha_{3}
\end{array}\right\} \\
& \alpha_{i} \quad \text { constantes a serem determinadas, } \\
& \mathrm{s} \quad \text { variável independente definida ao longo do } \\
& \text { lado ij do elemento( conforme figura 3.3). }
\end{aligned}
$$

Ao se aplicar a função anterior e sua derivada em relação a $s$, nos pontos i e j do lado $\mathrm{I}_{\mathrm{ij}}$, ter-se-á a relação seguinte : 


$$
\left\{\begin{array}{c}
w_{i} \\
w_{j} \\
w_{, s i} \\
w_{, j j}
\end{array}\right\}=\left[\begin{array}{cccc}
1 & 0 & 0 & 0 \\
1 & l_{i j} & l_{i j}^{2} & l_{i j}^{3} \\
0 & 1 & 0 & 0 \\
0 & 1 & 2 . l_{i j} & 3 . l_{i j}^{2}
\end{array}\right]\left\{\begin{array}{l}
\alpha_{0} \\
\alpha_{1} \\
\alpha_{2} \\
\alpha_{3}
\end{array}\right\}
$$

Da equação (3.41) obtém-se :

$$
\left\{\begin{array}{l}
\alpha_{0} \\
\alpha_{1} \\
\alpha_{2} \\
\alpha_{3}
\end{array}\right\}=\left[\begin{array}{cccc}
1 & 0 & 0 & 0 \\
0 & 0 & 1 & 0 \\
-\frac{3}{l_{i j}^{2}} & \frac{3}{l_{i j}^{2}} & -\frac{2}{l_{i j}} & -\frac{1}{l_{i j}} \\
\frac{2}{l_{i j}^{3}} & -\frac{2}{l_{i j}^{3}} & \frac{1}{l_{i j}^{2}} & \frac{1}{l_{i j}^{2}}
\end{array}\right]\left\{\begin{array}{c}
w_{i} \\
w_{j} \\
w_{, v i} \\
w_{., j}
\end{array}\right\}
$$

Assim, se obtém o deslocamento transversal $(w)$ ao longo do lado ij em função de $w_{i}, w_{, s i}, w_{j}, w_{, s j}$ como segue :

$w=\left(1-\frac{3 s^{2}}{l_{i j}^{2}}+\frac{2 s^{3}}{l_{i j}^{3}}\right) w_{i}+\left(s-\frac{2 s^{2}}{l_{i j}}+\frac{s^{3}}{l_{i j}^{2}}\right) w_{, s i}+\left(\frac{3 s^{2}}{l_{i j}^{2}}-\frac{2 s^{3}}{l_{i j}^{3}}\right) w_{j}+\left(-\frac{s^{2}}{l_{i j}}+\frac{s^{3}}{l_{i j}^{2}}\right) w_{, s j}$

onde $w_{s i}$ e $w_{s j}$ são as derivadas de $w_{s}$ nos ponto $i$ e j.

A derivada de $w$ em relação à variável $s$ é dada por:

$$
\begin{aligned}
w_{, s}=\left(-\frac{6 s}{l_{i j}^{2}}+\frac{6 s^{2}}{l_{i j}^{3}}\right) & w_{i}+\left(1-\frac{4 s}{l_{i j}}+\frac{3 s^{2}}{l_{i j}^{2}}\right) w_{, s i}+ \\
& \left(\frac{6 s}{l_{i j}^{2}}-\frac{6 s^{2}}{l_{i j}^{3}}\right) w_{j}+\left(-\frac{2 s}{l_{i j}}+\frac{3 s^{2}}{l_{i j}^{2}}\right) w_{, s j}
\end{aligned}
$$

A patir de (3.44) obtém-se a derivada de w em um ponto médio do lado $l_{i j}$, isto é : 
$w_{, s k}=-\left(3 /\left(21_{i j}\right)\right) w_{i}-(1 / 4) w_{, s i}+\left(3 /\left(21_{i j}\right)\right) w_{j}-(1 / 4) w_{, s j}$

$k$ ponto médio do lado ij.

Nesse elemento admite-se que a hipótese de Kirchhoff seja válida em todos os pontos nodais do contorno do elemento. Portanto, valem as relações seguintes :

$$
\begin{aligned}
& {[\gamma]=\left[\begin{array}{l}
\beta_{x}+w_{, x} \\
\beta_{y}+w_{, y}
\end{array}\right]=\left[\begin{array}{l}
0 \\
0
\end{array}\right] \quad \text { nos pontos } 1,2 \text { e } 3} \\
& \beta_{\text {sk }}+w_{\text {sk }}=0 \quad k=4,5,6
\end{aligned}
$$

Outra hipótese admitida para obtenção da matriz de rigidez do elemento é que $\beta_{n}$ varie linearmente ao longo dos lados, ou seja:

$$
\beta_{n k}=(1 / 2)\left(\beta_{n i}+\beta_{n j}\right),
$$

onde $k$ refere-se aos pontos 4,5 e 6 , para os lados 23,31 e 12, respectivamente.

Com base nas hipótese e relações anteriores, passa-se agora ao desenvolvimento da matriz de rigidez desse elemento. Para tanto a relação para $\beta_{x}$ e $\beta_{y}$ deve ser dada em função do vetor de parâmetros nodais dado na relação (3.34) .

Além disso, são válidas as seguintes relações geométricas no elemento triangular: 


$$
\left|\begin{array}{c}
\beta_{x} \\
\beta_{y}
\end{array}\right|=\left|\begin{array}{cc}
C & -S \\
S & C
\end{array}\right| \begin{gathered}
\beta_{n} \\
\beta_{s}
\end{gathered} \mid
$$

e

$$
\left|\begin{array}{l}
w_{w} \\
w_{, n}
\end{array}\right|=\left|\begin{array}{cc}
\mathrm{C} & \mathrm{S} \\
\mathrm{S} & -\mathrm{C}
\end{array}\right|\left|\begin{array}{l}
\theta_{\mathrm{x}} \\
\theta_{y}
\end{array}\right|
$$

$$
\text { onde } \mathrm{C}=\cos \left(\overrightarrow{\mathrm{x}}, \vec{n}_{i j}\right) \text { e } \mathrm{S}=\operatorname{sen}\left(\overrightarrow{\mathrm{x}}, \vec{n}_{i j}\right)
$$

e,

$$
\begin{aligned}
& w_{, x}=-\theta_{y} \\
& w_{, y}=\theta_{x}
\end{aligned}
$$

Assim seja a relação (3.38a) :

$$
\begin{gathered}
\beta_{x}=2(1-\xi-\eta)(1 / 2-\xi-\eta) \beta_{x 1}+\xi(2 \xi-1) \beta_{x 2}+\eta(2 \eta-1) \beta_{x 3}+ \\
4 \xi \eta \beta_{x 4}+4 \eta(1-\xi-\eta) \beta_{x 5}+4 \xi(1-\xi-\eta) \beta_{x 6}
\end{gathered}
$$

Da imposição da hipótese de Kirchhoff nos nós vem :

$$
\begin{aligned}
& \beta_{x 1}=\theta_{y 1} \\
& \beta_{x}=\theta_{y 2} \\
& \beta_{x}=\theta_{y 3}
\end{aligned}
$$

Das relações geométricas dadas em (3.48) e (3.49) pode-se escrever:

$$
\beta_{x}=\text { C. } \beta_{n}-S \cdot \beta_{s}
$$

onde $\mathrm{C}$ e $\mathrm{S}$ são os cossenos dos ângulos formados entre a normal aos lados, e o eixo X. Calculando $\beta_{x}$ para o nó 4 do elemento finito, obtém-se : 


$$
\beta_{x 4}=C_{23} \cdot \beta_{n 4}-S_{23} \cdot \beta_{s 4}
$$

Da hipótese dada na relação (3.47), tem-se que :

$$
\beta_{r .4}=(1 / 2)\left(\beta_{n 2}+\beta_{n 3}\right)
$$

E também que (hipótese de Kirchhoff) :

$$
\beta_{n}+w_{, n}=0 \Rightarrow \quad \beta_{n}=-w_{, n}
$$

Então :

$$
\begin{aligned}
& \beta_{n 2}=-w_{\text {,n2 }} \\
& \beta_{n 3}=-w_{, n 3}
\end{aligned}
$$

Ainda das relações geométricas (3.48) e (3.49) tem-se :

$$
w_{, n}=S \cdot \theta_{\mathrm{x}}-\mathrm{C} \theta_{\mathrm{y}}
$$

Portanto :

$$
\begin{aligned}
& w_{, n 2}=\mathrm{S}_{23} \cdot \theta_{\mathrm{x} 2}-\mathrm{C}_{23} \theta_{\mathrm{y} 2} \\
& w_{, n 3}=\mathrm{S}_{23} \cdot \theta_{\mathrm{x} 3}-\mathrm{C}_{23} \theta_{\mathrm{y} 3}
\end{aligned}
$$

Assim :

$$
\begin{aligned}
& \beta_{n 2}=-w_{, n 2}=-\left(S_{23} \cdot \theta_{x 2}-C_{23} \theta_{y 2}\right) \\
& \beta_{n 3}=-w_{, n 3}=-\left(S_{23} \cdot \theta_{x 3}-C_{23} \theta_{y 3}\right)
\end{aligned}
$$


Da imposição da hipótese de Kirchhoff nos nós do elemento, e da relação obtida da variação cúbica de $w$ ao longo dos lados, vem:

$$
\begin{aligned}
& \beta_{s k}=-w_{, s k} \Rightarrow \\
& \left.\beta_{s 4}=-w_{, s 4}=-\left(3 /\left(2 I_{23}\right)\right) w_{2}-(1 / 4) w_{, s 2}+\left(3 /\left(2 I_{23}\right)\right) w_{3}-(1 / 4) w_{, s 3}\right)
\end{aligned}
$$

Como :

$$
w_{, s}=C \cdot \theta_{x}+S \theta_{y}
$$

Logo ,

$$
\begin{aligned}
& w_{, s 2}=\mathrm{C}_{23} \cdot \theta_{\mathrm{x} 2}+\mathrm{S}_{23} \theta_{\mathrm{y} 2} \\
& w_{, s 3}=\mathrm{C}_{23} \cdot \theta_{\mathrm{x} 3}+\mathrm{S}_{23} \theta_{\mathrm{y} 3}
\end{aligned}
$$

Então, de (3.59) e (3.58) tem-se :

$$
\begin{aligned}
\beta_{s 4}= & -\left[3 /\left(2 \mathrm{I}_{23}\right)\right) w_{2}-(1 / 4)\left(\mathrm{C}_{23} \cdot \theta_{\mathrm{x} 2}+\mathrm{S}_{23} \theta_{\mathrm{y} 2}\right)+ \\
& \left.+\left(3 /\left(2 \mathrm{I}_{23}\right)\right) w_{3}-(1 / 4)\left(\mathrm{C}_{23} \cdot \theta_{\mathrm{x} 3}+\mathrm{S}_{23} \theta_{\mathrm{y}}\right)\right]
\end{aligned}
$$

A partir de (3.57) e (3.54) obtém-se $\beta_{n 4}$. Substituindo $\beta_{n 4}$ e $\beta_{s 4}$ (3.60) na relação para $\beta_{x 4}$, obter-se-á : 


$$
\begin{aligned}
\beta_{x 4}=C_{23}\{ & \left.\frac{1}{2}\left[\left(-S_{23} \theta_{x 2}+C_{23} \theta_{y 2}\right)+\left(-S_{23} \theta_{x 3}+C_{23} \theta_{y 3}\right)\right]\right\}- \\
& -S_{23}\left[\frac{3}{2 l_{23}} w_{2}-\frac{1}{4}\left(C_{23} \theta_{x 2}+S_{23} \theta_{y 2}\right)-\frac{3}{2 l_{23}} w_{3}+\frac{1}{4}\left(C_{23} \theta_{x 3}+S_{23} \theta_{y 3}\right.\right.
\end{aligned}
$$

Adotando o mesmo procedimento para $\beta_{\times 5}$ e $\beta_{\times 6}$ ter-se-á :

$$
\begin{aligned}
& \beta_{x 5}=C_{31}\left\{\frac{1}{2}\left[\left(-S_{31} \theta_{x 3}+C_{31} \theta_{y 3}\right)+\left(-S_{31} \theta_{x 1}+C_{31} \theta_{y 1}\right)\right]\right\}- \\
& -\mathrm{S}_{31}\left[\frac{3}{21_{31}} \mathrm{w}_{3}-\frac{1}{4}\left(\mathrm{C}_{31} \theta_{\mathrm{x} 3}+\mathrm{S}_{31} \theta_{\mathrm{y} 3}\right)-\frac{3}{21_{31}} \mathrm{w}_{1}+\frac{1}{4}\left(\mathrm{C}_{31} \theta_{\mathrm{x} 1}+\mathrm{S}_{31} \theta_{\mathrm{y1}}\right)\right]
\end{aligned}
$$

e

$$
\begin{aligned}
\beta_{\mathrm{x} 6}= & \mathrm{C}_{12}\left\{\frac{1}{2}\left[\left(-\mathrm{S}_{12} \theta_{\mathrm{x} 1}+\mathrm{C}_{12} \theta_{\mathrm{y} 1}\right)+\left(-\mathrm{S}_{12} \theta_{\mathrm{x} 2}+\mathrm{C}_{12} \theta_{\mathrm{y} 2}\right)\right]\right\}- \\
& -\mathrm{S}_{12}\left[\frac{3}{21_{12}} \mathrm{w}_{1}-\frac{1}{4}\left(\mathrm{C}_{12} \theta_{\mathrm{x} 1}+\mathrm{S}_{12} \theta_{\mathrm{y} 1}\right)-\frac{3}{21_{12}} \mathrm{w}_{2}+\frac{1}{4}\left(\mathrm{C}_{12} \theta_{\mathrm{x} 2}+\mathrm{S}_{12} \theta_{\mathrm{y} 2}\right)\right]
\end{aligned}
$$

Ao se substituir $\beta_{x 1}, \beta_{x 2}, \beta_{x 3}, \beta_{x 4}, \beta_{x 5}$ e $\beta_{x 6}$ em $\beta_{x}$, ter-se-á este último dados em função dos parâmetros nodais. $O$ mesmo procedimento é adotado para $\beta_{y}$. As relações resultantes são dadas a seguir.

$$
\begin{aligned}
& \beta_{x}=H_{x}^{\top}(\xi, \eta) \mathbf{u} \\
& \beta_{y}=H_{y}^{\top}(\xi, \eta) \mathbf{u}
\end{aligned}
$$

onde $u$ é o vetor de parâmetros nodais dado por (3.34), e $H_{x}$ e $H_{y}$ são as nove componentes do vetor função de forma. As componentes são funções de $N_{i}, \mathrm{i}=1,6$ e das coordenadas dos nós, 


$$
\begin{aligned}
& \mathbf{H}^{\top}{ }_{\mathbf{x}}(\xi, \eta)=\left\langle\begin{array}{lllllllllll}
H_{x 1} & H_{x 2} & H_{x 3} & H_{x 4} & H_{x 5} & H_{x 6} & H_{x 7} & H_{x 8} & H_{x 9}
\end{array}\right\rangle \\
& \mathbf{H}_{\mathrm{y}}^{\top}(\xi, \eta)=\left\langle\begin{array}{llllllllll}
\mathrm{H}_{\mathrm{y} 1} & \mathrm{H}_{\mathrm{y} 2} & \mathrm{H}_{\mathrm{y} 3} & \mathrm{H}_{\mathrm{y} 4} & \mathrm{H}_{\mathrm{y} 5} & \mathrm{H}_{\mathrm{y} 6} & \mathrm{H}_{\mathrm{y} 7} & \mathrm{H}_{\mathrm{y} 8} & \mathrm{H}_{\mathrm{y} 9}
\end{array}\right\rangle
\end{aligned}
$$

As funções $H_{x i}$ e $H_{y i}$ são dadas a seguir :

$$
\begin{aligned}
& \mathrm{H}_{\mathrm{x} 1}=1.5\left(\mathrm{a}_{6} \mathrm{~N}_{6}-\mathrm{a}_{5} \mathrm{~N}_{5}\right) \\
& \mathrm{H}_{\mathrm{x} 2}=\mathrm{b}_{5} \mathrm{~N}_{5}+\mathrm{b}_{6} \mathrm{~N}_{6} \\
& \left.\mathrm{H}_{\mathrm{x} 3}=\mathrm{N}_{1}-\mathrm{c}_{5} \mathrm{~N}_{5}-\mathrm{c}_{6} \mathrm{~N}_{6}\right) \\
& \mathrm{H}_{\mathrm{y} 1}=1.5\left(\mathrm{~d}_{6} \mathrm{~N}_{6}-\mathrm{d}_{5} \mathrm{~N}_{5}\right) \\
& \mathrm{H}_{\mathrm{y} 2}=-\mathrm{N}_{1}+\mathrm{e}_{5} \mathrm{~N}_{5}+\mathrm{e}_{6} \mathrm{~N}_{6} \\
& \mathrm{H}_{\mathrm{y} 3}=-\mathrm{H}_{\mathrm{x} 2}
\end{aligned}
$$

As funções $\mathrm{H}_{\mathrm{x} 4}, \mathrm{H}_{\mathrm{x} 5}, \mathrm{H}_{\mathrm{x} 6}, \mathrm{H}_{\mathrm{y} 4}, \mathrm{H}_{\mathrm{y} 5}$ e $\mathrm{H}_{\mathrm{y} 6}$ são obtidas das expressões acima trocando $N_{1}$ por $N_{2}$ e os índices 6 e5 por 4 e 6 , respectivamente. As funçōes $H_{x 7}, H_{x 8}, H_{x 9}, H_{y 7}, H_{y 8}$ e $H_{y 9}$ são obtidas trocando $N_{1}$ por $N_{3}$ e os índices 6 e 5 por 5 e 4 , respectivamente.Tem-se também as seguintes relações :

$$
\begin{aligned}
& a_{k}=-x_{i j} / l_{i j}^{2} \\
& b_{k}=(3 / 4) x_{i j} y_{i j} / /_{i j}^{2} \\
& c_{k}=\left((1 / 4) x_{i j}{ }^{2}-(1 / 2) y_{i j}{ }^{2}\right) / l_{i j}{ }^{2} \\
& d_{k}=-y_{i j} / l_{i j}^{2} \\
& e_{k}=\left((1 / 4) y_{i j}{ }^{2}-(1 / 2) x_{i j}{ }^{2}\right) / l_{i j}{ }^{2} \\
& l_{i j}{ }^{2}=\left(x_{i j}{ }^{2}+y_{i j}{ }^{2}\right)
\end{aligned}
$$

onde $k=4,5,6$ para os lados $i j=23,31,12$ respectivamente.

A partir de (3.64), o vetor de curvaturas dado por (3.23) pode ser reescrito na seguinte forma: 


$$
k=B \cdot u
$$

onde B é a matriz dada a seguir :

$$
\mathbf{B}(\xi, \eta)=\frac{1}{2 A}\left[\begin{array}{c}
y_{31} \mathbf{H}_{x, \xi}^{\mathrm{T}}+y_{12} \mathbf{H}_{x, \eta}^{\mathrm{T}} \\
-x_{31} \mathbf{H}_{y, \xi}^{\mathrm{T}}-x_{12} \mathbf{H}_{y, \eta}^{\mathrm{T}} \\
-x_{31} \mathbf{H}_{x, \xi}^{\mathrm{T}}-x_{12} \mathbf{H}_{x, \eta}^{\mathrm{T}}+y_{31} \mathbf{H}_{y, \xi}^{\mathrm{T}}+y_{12} \mathbf{H}_{y, \eta}^{\mathrm{T}}
\end{array}\right]
$$

onde:

$$
2 A=x_{31} y_{12}-x_{12} y_{31} \text {. }
$$

u é o vetor de parâmetros nodais.

Partindo das definições para $\mathrm{H}_{x}$ e $\mathrm{H}_{y}$ e das funções de forma $\mathrm{N}_{i}$ pode-se obter as suas derivadas com relação a $\xi$ e $\eta$, que são dadas a seguir:

$$
\mathbf{H}_{x, 5}=\left[\begin{array}{c}
\mathrm{P}_{6}(1-2 \xi)+\left(\mathrm{P}_{5}-\mathrm{P}_{6}\right) \eta \\
\mathrm{q}_{6}(1-2 \xi)-\left(\mathrm{q}_{5}+\mathrm{q}_{6}\right) \eta \\
-4+6(\xi+\eta)+\mathrm{r}_{6}(1-2 \xi)-\eta\left(\mathrm{r}_{5}+\mathrm{r}_{6}\right) \\
-\mathrm{P}_{6}(1-2 \xi)+\eta\left(\mathrm{P}_{4}+\mathrm{P}_{6}\right) \\
\mathrm{q}_{6}(1-2 \xi)-\eta\left(\mathrm{q}_{6}-\mathrm{q}_{4}\right) \\
-2+6 \xi+\mathrm{r}_{6}(1-2 \xi)+\eta\left(\mathrm{r}_{4}-\mathrm{r}_{6}\right) \\
-\eta\left(\mathrm{P}_{5}+\mathrm{P}_{4}\right) \\
\eta\left(\mathrm{q}_{4}-\mathrm{q}_{5}\right) \\
-\eta\left(\mathrm{r}_{5}-\mathrm{r}_{4}\right)
\end{array}\right]
$$


$\mathbf{H}_{\mathrm{y}, \xi}=\left[\begin{array}{c}\mathrm{t}_{6}(1-2 \xi)+\eta\left(\mathrm{t}_{5}-\mathrm{t}_{6}\right) \\ 1+\mathrm{r}_{6}(1-2 \xi)-\eta\left(\mathrm{r}_{5}+\mathrm{r}_{6}\right) \\ -\mathrm{q}_{6}(1-2 \xi)+\eta\left(\mathrm{q}_{5}+\mathrm{q}_{6}\right) \\ -\mathrm{t}_{6}(1-2 \xi)+\eta\left(\mathrm{t}_{4}+\mathrm{t}_{6}\right) \\ -1+\mathrm{r}_{6}(1-2 \xi)+\eta\left(\mathrm{r}_{4}-\mathrm{r}_{6}\right) \\ -\mathrm{q}_{6}(1-2 \xi)-\eta\left(\mathrm{q}_{4}-\mathrm{q}_{6}\right) \\ -\eta\left(\mathrm{t}_{4}+\mathrm{t}_{5}\right) \\ \eta\left(\mathrm{r}_{4}-\mathrm{r}_{5}\right) \\ -\eta\left(\mathrm{q}_{4}-\mathrm{q}_{5}\right)\end{array}\right]$

$\mathbf{H}_{x, \eta}=\left[\begin{array}{c}-P(1-2 \eta)-\xi\left(P_{6}-P_{5}\right) \\ q_{5}(1-2 \eta)-\xi\left(q_{5}+q_{6}\right) \\ -4+6(\xi+\eta)+r_{5}(1-2 \eta)-\xi\left(r_{5}+r_{6}\right) \\ \xi\left(P_{4}+P_{6}\right) \\ \xi\left(q_{4}-q_{6}\right) \\ -\xi\left(r_{6}-r_{4}\right) \\ P_{5}(1-2 \eta)-\xi\left(P_{4}+P_{5}\right) \\ q_{5}(1-2 \eta)+\xi\left(q_{4}-q_{5}\right) \\ -2+6 \eta+r_{5}(1-2 \eta)+\xi\left(r_{4}-r_{5}\right)\end{array}\right]$

$$
\mathbf{H}_{y, \eta}=\left[\begin{array}{c}
-t_{5}(1-2 \eta)-\xi\left(t_{6}-t_{5}\right) \\
1+r_{5}(1-2 \eta)-\xi\left(r_{5}+r_{6}\right) \\
-q_{5}(1-2 \eta)+\xi\left(q_{5}+q_{6}\right) \\
\xi\left(t_{4}+t_{6}\right) \\
\xi\left(r_{4}-r_{6}\right) \\
-\xi\left(q_{4}-q_{6}\right) \\
t_{5}(1-2 \eta)-\xi\left(t_{4}+t_{5}\right) \\
-1+r_{5}(1-2 \eta)+\xi\left(r_{4}-r_{5}\right) \\
-q_{5}(1-2 \eta)-\xi\left(q_{4}-q_{5}\right)
\end{array}\right]
$$


onde:

$$
\begin{aligned}
& P_{k}=-6 x_{i j} / l_{i j}^{2}=6 a_{k} \\
& q_{k}=3 x_{i j} y_{i j} / l_{i j}^{2}=4 b_{k} \\
& t_{k}=-6 y_{i j} / l_{i j}^{2}=6 d_{k} \\
& r_{k}=3 y_{i j}^{2} /_{i j}^{2} \\
& k=4,5,6 \text { para ij }=23,31,12 \text { respectivamente. }
\end{aligned}
$$

O processo para se obter a matriz de rigidez segue o procedimento básico do método dos elementos finitos, minimizando a energia potencial

Substituindo as relações já obtidas na expressão da energia de deformação, podemos chegar na matriz de rigidez :

$$
\mathbf{K}_{\text {DKT }}=2 A \int_{0}^{1} \int_{0}^{1-\eta} \mathbf{B}^{\mathbf{T}} \mathbf{D}_{\mathbf{b}} \mathbf{B} d \xi d \eta
$$

Consegue-se dessa forma obter a matriz de rigidez desejada. Para se resolver a integral anterior, usa-se as regras básicas de integração de funções em domínios triangulares. Pode-se agora, passar à montagem da matriz de rigidez do elemento de casca plano. 


\section{O ELEMENTO FINITO DE CASCA PLANO}

\subsection{INTRODUÇÃO}

Nesse capítulo monta-se o elemento finito de casca plano, usando os elementos finitos que se tem desenvolvido. Esse elemento finito é obtido pela adição da rigidez dos elementos de membrana e de placa. Entretanto, adicionar as matrizes de rigidez, não significa que se fará uma soma aigébrica simplesmente. Trata-se de alocar os coeficientes de rigidez conforme disposição do vetor de parâmetros nodais do elemento de casca, definido a partir dos elementos de membrana e de placa

$O$ procedimento de montagem da matriz de rigidez do elemento de casca plano é o mesmo apresentado em ZIENKIEWICZ(1977), e será exposto nos itens seguintes, seguifhdo os mesmos passos apresentados na referência mencionada.

\subsection{RIGIDEZ DE UM ELEMENTO DE CASCA PLANO EM COORDENADAS LOCAIS}

Considere um elemento finito plano submetido concomitantemente às ações paralelas e perpendiculares ao seu plano médio, conforme figura 4.1: 

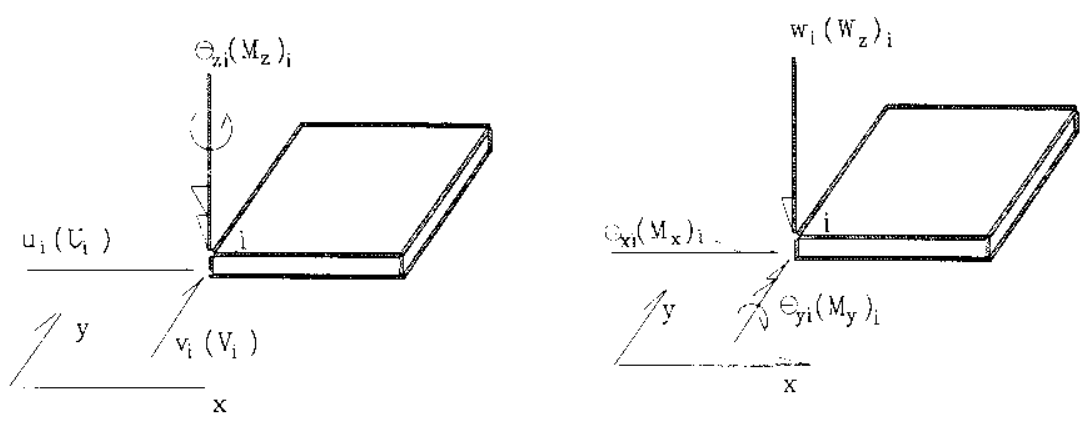

Figura 4.1 Elemento finito plano( apud ZIENKIEWICZ 1977).

Para as ações paralelas a seu plano, o estado de deformação é descrito unicamente em função dos desiocamentos $u$ e $v$, segundo o plano cartesiano $X Y$, respectivamente. A matriz de rigidez foi obtida minimizando a energia potencial, sendo válida a relação seguinte :

$$
K^{m} \cdot a^{m}=f^{m}
$$

onde :

$\mathbf{a}^{m}{ }_{i}$ é o vetor de parâmetros nodais para ações de membrana,

$\mathbf{f}^{\mathrm{m}}{ }_{\mathrm{i}}$ é o vetor de forças nodais atuantes na membrana,

$\mathrm{K}^{\mathrm{m}}{ }_{\mathrm{i}}$ é a matriz de rigidez associada ao vetor de parâmetros nodais da membrana.

sendo :

$$
a_{i}^{m}=\left\langle\begin{array}{c}
u_{i} \\
v_{i}
\end{array}\right\rangle \quad \text { e } f_{i}^{m}=\left\langle\begin{array}{l}
F_{x i} \\
F_{y i}
\end{array}\right\rangle
$$


Da mesma forma, para as ações de flexão, o estado de deformação vem expresso em função dos deslocamentos na direção $Z(w)$ e das rotaçöes $\theta_{x}$ e $\theta_{y}$. Portanto :

$$
K^{e b} \cdot a^{\frac{k}{m}}=f^{e b}
$$

onde:

$\mathbf{a}^{\mathrm{b}}{ }_{\mathrm{i}}$ é o vetor de parâmetros nodais.

$f_{i}^{b}$ é o vetor de forças nodais.

$\mathbf{K}^{\mathrm{eb}}$ é a parcela da matriz de rigidez associada ao vetor de parâmetros nodais $\mathbf{a}_{i}{ }_{i}$,

sendo :

$$
a_{i}^{b}=\left\{\begin{array}{c}
w_{i} \\
\theta_{x i} \\
\theta_{y i}
\end{array}\right\} \quad e, \quad f_{i}^{b}=\left\{\begin{array}{c}
F_{z i} \\
M_{x i} \\
M_{y i}
\end{array}\right\}
$$

Deve-se agora ressaltar dois importantes aspectos na formulação do elemento de casca plano. O primeiro, é que os deslocamentos impostos para forças paralelas ao plano médio do elemento não alteram as deformações de flexão e vice-versa. A segunda, que o giro $\theta_{z}$ não interfere como parâmetro de definição das deformações em nenhum dos casos. Com relação ao giro $\theta_{z}$,será introduzido na montagem da matriz como rigidez fictícia, e sua justificativa será feita no próximo ítem.

Assim, os parâmetros nodais combinados são dados a seguir :

$$
K^{e} \cdot a=f^{e}
$$

onde :

$a_{i}$ é o vetor de parâmetros nodais do elemento de casca plano, $f_{i}^{e}$ é o vetor de forças nodais atuantes no elemento de casca,

$\mathbf{K}_{\mathrm{i}}^{\mathrm{e}}$ é a parcela da matriz de rigidez do elemento de casca pla no, associada ao vetor $\mathbf{a}_{i}$ 
sendo:

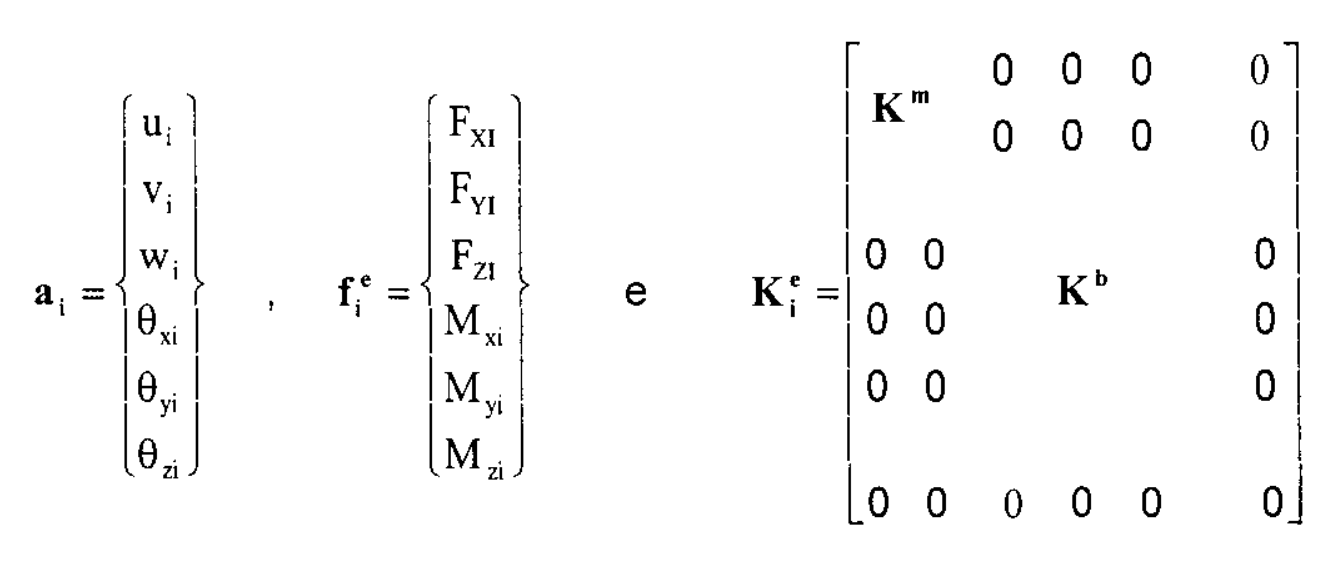

Note-se que essa rigidez refere-se a um sistema de coordenadas locais, ou em um mesmo plano. Quando se trabaha em planos distintos, para se adicionar a rigidez de todos os elementos, deve-se compatibilizar o sistema de referência.

\subsection{ELEMENTO FINITO DE CASCA PLANO}

Usando os elementos finitos já vistos nos capítulos II e III, e seguindo os passos dados nos ítens anteriores, pode-se montar o elemento finito de casca plano, que é o intuito desse trabalho. Esse elemento está mostrado na figura 4.2 


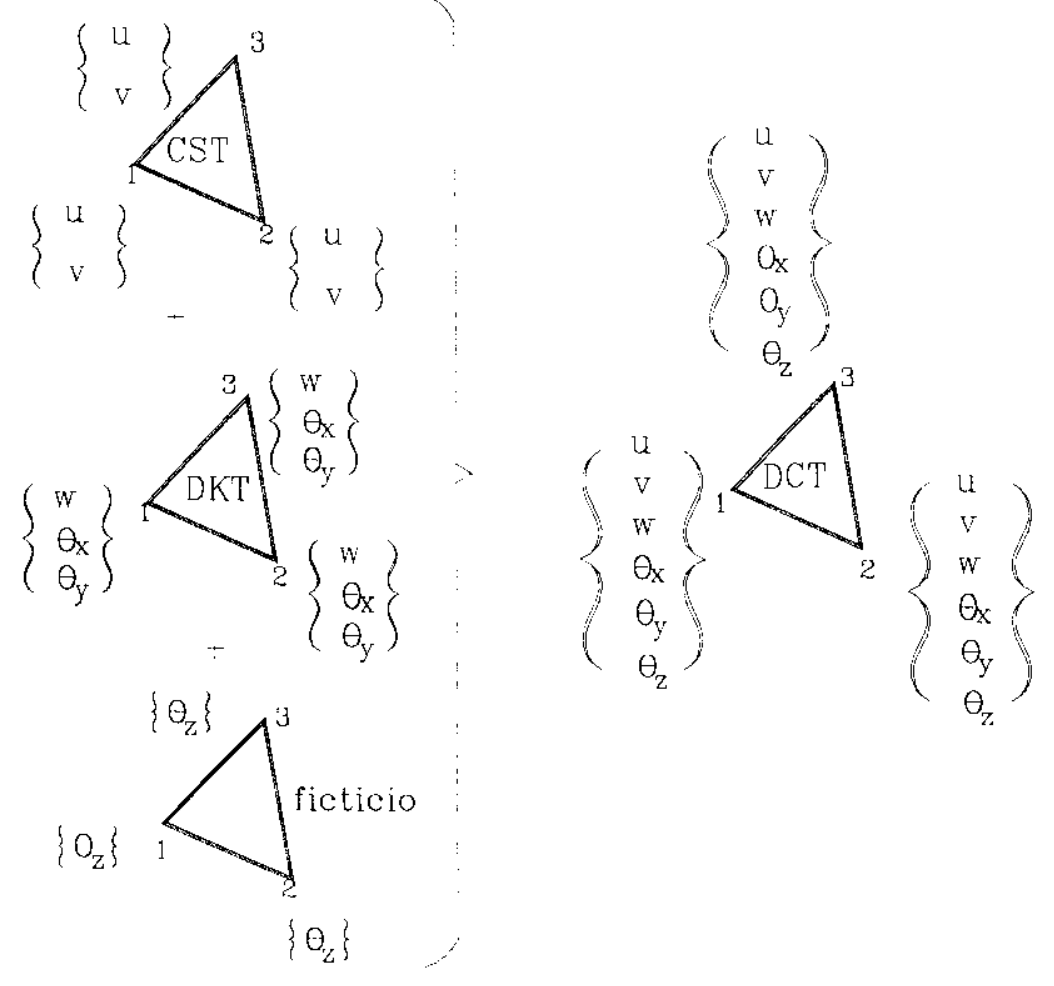

Figura 4.2 Elemento finito de casca plano.

Assim a matriz de rigidez do elemento de casca será dada por:

$$
k_{\mathrm{dct}}=k_{\mathrm{cst}}+k_{\mathrm{dkt}}+k_{\theta z}
$$

A adição representada na última equação refere-se ao acoplamento dos elementos que compõem o elemento finito de casca plano, e não de uma adição matricial, como poderia se supor. $O$ procedimento para se montar essa matriz de rigidez será mostrado em seguida.

O vetor de parâmetros nodais é dado por :

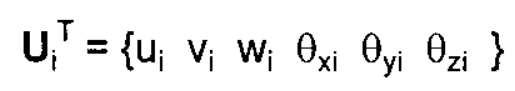

Neste caso, tem-se um elemento finito com 6 graus de liberdade por nó, totalizando um elemento com 18 graus de liberdade. 


\subsection{RIGIDEZ ROTACIONAL FICTÍCIA.}

Nessa formulação adotada aparece uma dificuldade quando todos os elementos que concorrem em um mesmo ponto são coplanares. Isso ocorre porque impõe-se um valor nulo à rigidez na direção $\theta_{z}$.

Se nesse ponto considerando-se o sistema das equações de equilíbrio em coordenadas locais, ter-se-á seis equações, a última das quais é apenas:

$$
0=0
$$

Para resolver esse problema, adota-se nesses pontos um coeficiente de rigidez arbitrário $\left(k_{\theta z}^{\prime}\right)$. Isso conduz a resolver a equação :

$$
k_{\theta z i}^{\prime} \cdot \theta_{z i}=0
$$

Como $\theta_{z i}$ não altera as tensōes, e por consequência não interfere em nehuma equação de equilibrio, pode-se adotar para $k_{\theta z i}^{\prime}$ qualquer valor arbitrário, pois não afeta o resultado. Neste trabalho tem-se adotado o valor 0.5 .

\subsection{TRANSFORMAÇÃO PARA COORDENADAS GLOBAIS}

A matriz de rigidez deduzida na seção anterior utilizava um sistema de coordenadas locais. Para montar a matriz de rigidez da estrutura, precisa-se trabalhar com um sistema de coordenadas compatíveis, já que cada 
elemento de casca estará vinculado a seu próprio sistema de referência( sistema local, cujas coordenadas passa-se a designar por $x^{\prime}, y^{\prime}$ e z'). A esse sistema de coordenadas compativeis chama-se de sistema global de coordenadas ( coordenadas $x, y$ e $z$ ). Isso permite que se tenha todas as matrizes relacionadas a um mesmo sistema de referência, onde se determinará os deslocamentos nodais.

Todos os pontos nodais do elementos terão suas coordenadas definidas no sistema global e, de forma conveniente, faz-se as transformações para se encontrar as coordenadas locais de cada elemento. Assim, calculase todas as matrizes de rigidez no sistema local, e se faz a transformação para o sistema global, onde monta-se a matriz de rigidez da estrutura. Impondo as condições de contorno no sistema global, pode-se resolver o sistema de equações lineares e obter os deslocamentos desejados.

\subsubsection{TRANSFORMAÇÃO DE COORDENADAS LOCAIS EM UM SISTEMA QUALQUER}

De um modo geral, os vetores de parâmetros nodais de um nó de um elemento finito, em relação aos sistemas de coordenadas global e local, estão relacionados por :

$$
\mathbf{a}^{\prime}=\text { L.a }
$$

onde:

L é a matriz dos cossenos diretores adequada,

a é o vetor de parâmetros nodais no sistema global,

a' é o vetor de parâmetros nodais no sistema local. 
As correspondentes componentes das forças devem realizar a mesma quantidade de trabalho, portanto :

$$
q^{\top} \cdot a=q^{\prime \top} \cdot a^{\prime}
$$

q vetor de forças nodais quaisquer.

Das relações (4.5) e (4.6) tem-se :

$$
q^{\top} \cdot a=q^{\prime \top} \cdot L \cdot a
$$

ou seja :

$$
q=L^{\top} \cdot q^{\prime}
$$

Pode-se escrever a relação :

$$
q^{\prime}=K^{\prime} \cdot a^{\prime}
$$

K' é a matriz de rigidez no sistema local de coordenadas.

Assim tem-se :

$$
q=L^{\top} K^{\prime} L a
$$

Como:

$$
\mathbf{q}=\mathrm{K} . \mathbf{a}
$$

K é a matriz de rigidez no sistema global de coordenadas. 
Finalmente, em coordenadas globais ter-se-á :

$$
K=L^{\top} K^{\prime} L
$$

Assim, a partir da matriz de rigidez no sistema local, pode-se obter sua transformação para o sistema global. Usa-se a expressão anterior para transportarmos todas as matrizes para o mesmo sistema de referência ( global ), onde monta-se a matriz de rigidez da estrutura.

\subsubsection{MONTAGEM DA MATRIZ NO SISTEMA GLOBAL}

$\mathrm{Na}$ figura 4.3 tem-se representados os dois sistemas de referência, local ( $x^{\prime}, y^{\prime}$ e $z^{\prime}$ ) e global ( $x, y$ e $z$ ). Como já foi visto, as forças e os deslocamentos de um nó se transformam de um sistema global ao local por uma matriz $L$ da forma como segue:

$$
a_{i}^{\prime}=L a_{i} \quad ; \quad f_{i}^{\prime}=L f_{i}
$$

onde:

$$
\mathbf{L}=\left[\begin{array}{ll}
\lambda & 0 \\
0 & \lambda
\end{array}\right]
$$

sendo $\lambda$ a matriz dos cossenos diretores de dimensão $3 \times 3$, dos ângulos que formam entre si os dois sistemas de eixo, ou seja:

$$
\lambda=\left[\begin{array}{lll}
\lambda_{x^{\prime} x} & \lambda_{x^{\prime} y} & \lambda_{x^{\prime} z} \\
\lambda_{y^{\prime} x} & \lambda_{y^{\prime} y} & \lambda_{y^{\prime} z} \\
\lambda_{z^{\prime} x} & \lambda_{z^{\prime} y} & \lambda_{z^{\prime} z}
\end{array}\right]
$$

onde : $\quad \lambda_{x^{\prime} x}=$ cosseno do ângulo formado pelos eixos $x$ e $x^{\prime}$. 


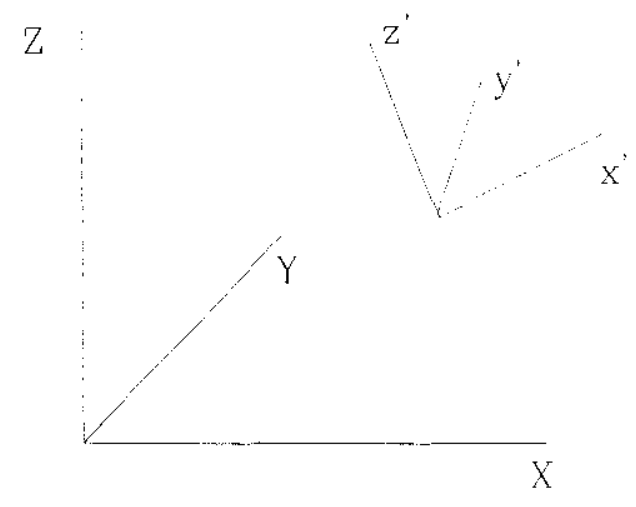

Figura 4.3 Sistemas global e local( apud ZIENKIEWICZ, 1977).

Portanto para o conjunto de forças que atuam sobre os nós do elemento pode-se escrever :

$$
a^{, e}=T a^{e},
$$

onde :

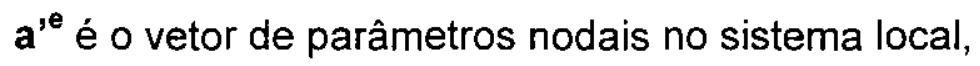

$\mathbf{a}^{\mathbf{e}}$ é o vetor de parâmetros nodais no sistema global,

T é a matriz de transformação dada por:

$$
\mathbf{T}=\left[\begin{array}{lll}
\mathbf{L} & \mathbf{0} & \mathbf{0} \\
\mathbf{0} & \mathbf{L} & \mathbf{0} \\
\mathbf{0} & \mathbf{0} & \mathbf{L}
\end{array}\right]
$$

Segundo as regras das transformações ortogonais dadas na seção anterior, a matriz de rigidez de um elemento em coordenadas globais é dada como segue :

$$
K^{e}=T^{\top} \cdot K^{, e} \cdot T
$$

Para um elemento com maior quantidade de nós, basta usar na diagonal da matriz $T$ um número de matrizes $L$ igual à quantidade de nós do 
elemento que se está usando. As coordenadas locais serão calculadas como segue, se a origem dos sistema de eixos coincide :

$$
\left\{\begin{array}{l}
x^{\prime} \\
y^{\prime} \\
z^{\prime}
\end{array}\right\}=\lambda\left\{\begin{array}{l}
x \\
y \\
z
\end{array}\right\}
$$

onde $\lambda$ é a matriz dos cossenos diretores.

\subsubsection{MATRIZ DOS COSSENOS DIRETORES}

Seja uma casca dada na figura 4.4 dividida em elementos triangulares. Representa-se por ijm um triângulo qualquer. Tomando arbitrariamente o lado ij como uma das direções do sistema local ( $x^{\prime}$ ), pode-se encontrar a matriz dos cossenos diretores.

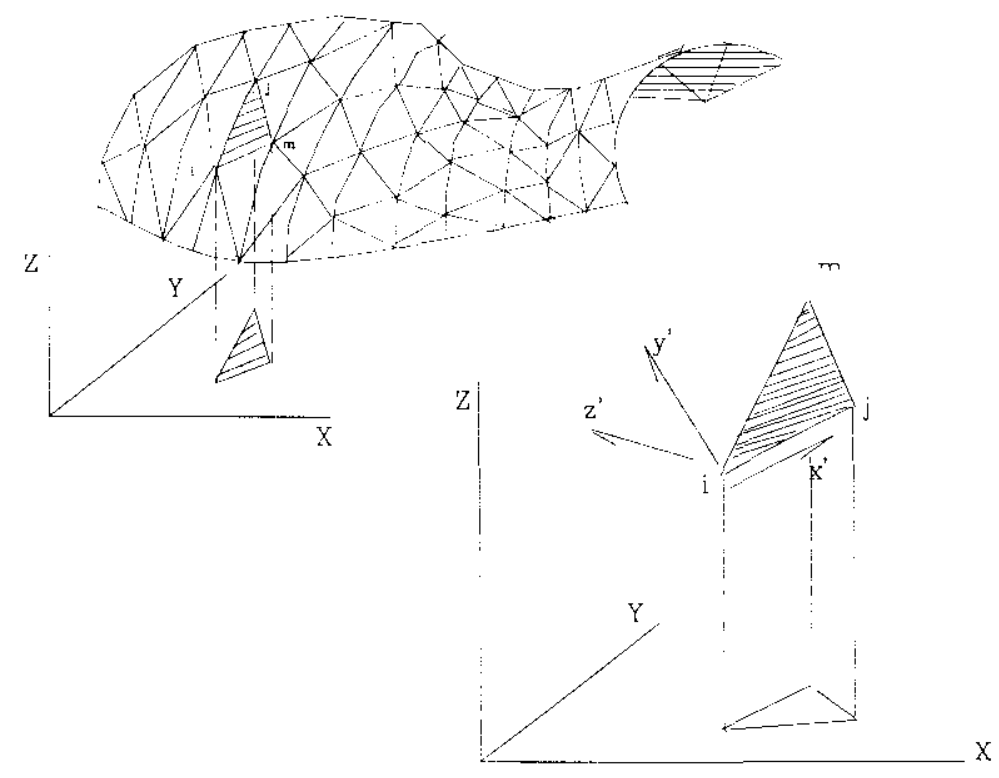

Figura 4.4 Casca dividida em elementos triangulares planos( apud ZIENKIEWICZ, 1977). 
Esse lado está definido pelo vetor $\mathbf{V}_{\mathrm{ij}}$ e em função das coordendas globais . Portanto :

$$
\mathbf{V}_{i j}=\left\{\begin{array}{c}
x_{j}-x_{i} \\
y_{j}-y_{i} \\
z_{j}-z_{i}
\end{array}\right\}
$$

Os cossenos diretores obtém-se dividindo as componente deste vetor por seu módulo, ou seja definindo o vetor unitário :

$$
\begin{aligned}
& \mathbf{v}_{x^{\prime}}=\left\{\begin{array}{l}
\lambda_{x^{\prime} x} \\
\lambda_{x^{\prime} y} \\
\lambda_{x^{\prime} z}
\end{array}\right\}=\frac{1}{l_{i j}}\left\{\begin{array}{l}
x_{i j} \\
y_{i j} \\
z_{i j}
\end{array}\right\} \\
& \text { onde : } \quad l_{i j}=\sqrt{x_{i j}^{2}+y_{i j}^{2}+z_{i j}^{2}} \\
& x_{\mathrm{ij}}=\mathrm{x}_{\mathrm{j}}-\mathrm{x}_{\mathrm{i}}
\end{aligned}
$$

A direção $z^{\prime}$ deve ser normal ao plano do triângulo. Fazendo o produto vetorial dos vetores associados aos lados $i j \mathrm{e} i m$ do triângulo dado, obtém-se o vetor $V_{z^{\prime}}$ que é perpendicular ao plano do triângulo. Assim sendo,

$$
\mathbf{V}_{z^{\prime}}=\mathbf{V}_{\mathrm{ij}} \mathrm{xV}_{\mathrm{im}}=\left\{\begin{array}{l}
\mathrm{y}_{\mathrm{ij}} \mathrm{z}_{\mathrm{im}}-\mathrm{z}_{\mathrm{ij}} \mathrm{y}_{\mathrm{im}} \\
\mathrm{z}_{\mathrm{ij}} \mathrm{x}_{\mathrm{im}}-\mathrm{x}_{\mathrm{ij}} \mathrm{z}_{\mathrm{ji}} \\
\mathbf{x}_{\mathrm{ij}} \mathrm{y}_{\mathrm{im}}-\mathrm{y}_{\mathrm{ij}} \mathrm{x}_{\mathrm{im}}
\end{array}\right\}
$$

onde, $x_{i j}=x_{j}-x_{i}$, etc.

O módulo desse vetor é dado por:

$1_{z^{\prime}}=\sqrt{\left(y_{i j} z_{i m}-z_{i j} y_{i m}\right)^{2}+\left(z_{i j} x_{i m}-x_{i j} z_{j i}\right)^{2}+\left(x_{i j} y_{i m}-y_{i j} x_{i m}\right)^{2}}=2 . A$

sendo $\mathrm{A}$ a área do triângulo. 
Os cossenos diretores serão dados pelo vetor unitário na direção z'. Ou seja :

$$
\mathbf{v}_{\mathbf{z}}^{\prime}=\left\{\begin{array}{c}
\lambda_{z^{\prime} x} \\
\lambda_{z^{\prime} y} \\
\lambda_{z^{\prime} z}
\end{array}\right\}=\frac{\mathbf{V}_{z}^{\prime}}{l_{z^{\prime}}}
$$

Finalmente, os cossenos diretores do eixo y' serão obtidos pelo produto vetorial dos vetores unitários $\mathbf{v}_{z^{\prime}}$ e $\mathbf{v}_{\mathrm{x}^{\prime}}$, ou seja :

$$
\mathbf{v}_{y^{\prime}}=\mathbf{v}_{z^{\prime}} x \mathbf{v}_{x^{\prime}}=\left\{\begin{array}{l}
\lambda_{y^{\prime} x} \\
\lambda_{y^{\prime} y} \\
\lambda_{y^{\prime} z}
\end{array}\right\}=\left\{\begin{array}{l}
\lambda_{z^{\prime} y} \lambda_{x^{\prime} z}-\lambda_{y^{\prime} z} \lambda_{x^{\prime} y} \\
\lambda_{y^{\prime} z} \lambda_{x^{\prime} x}-\lambda_{z^{\prime} x} \lambda_{x^{\prime} z} \\
\lambda_{y^{\prime} x} \lambda_{x^{\prime} y}-\lambda_{z^{\prime} y} \lambda_{x^{\prime} x}
\end{array}\right\}
$$

Não se divide esse vetor pelo seu módulo, pois se trata do produto vetorial de dois vetores unitários.

Tem-se, finalmente, encontrado a matriz dos cossenos diretores. Substituindo-a na matriz $T$, pode-se proceder as transformações que se tem referido. 


\section{O PROGRAMA E EXEMPLOS NUMÉRICOS.}

\subsection{INTRODUÇÃO}

O programa foi elaborado em linguagem FORTRAN, fazendo-se uso do compilador Microsoft Power Station 1.0. Consta de cinco módulos independentes, onde a comunicação entre eles é feita por meio de arquivo de dados. Usa-se arquivos binários, pois além de facilitar, utilizam um tempo de leitura menor.

No primeiro módulo do programa faz-se a leitura dos dados. A entrada de dados também é feita via arquivo. No módulo seguinte monta-se a matriz de rigidez de cada elemento já no sistema global de coordenadas, gravando cada matriz num arquivo de dados auxiliar. Cada matriz será gravada em um registro diferente. Isso evita que o próximo módulo faça leitura equivocada. O terceiro módulo é responsável pela montagem da matriz de rigidez da estrutura, processando a leitura das matrizes de cada elemento calculadas no módulo II e alocando os coeficientes na matriz de rigidez da estrutura. Também são impostas as condições de contorno da estrutura na montagem final da matriz da estrutura.

Após leitura da matriz de rigidez da estrutura, e do respectivo vetor de forças nodais do módulo III, o quarto módulo resolve o sistema de equações 
lineares. A resolução desse sistema nos fornece os deslocamentos nodais da malha usada. Esses deslocamentos são dados em coordenadas globais.

Finalmente pode-se encontrar os esforços atuantes na estrutura. Isso será feito no quinto módulo. Antes, porém, deve-se encontrar os deslocamentos em coordenadas locais. Com base no que foi exposto no capitulo IV, pode-se fazer essa transformação. Para encontrar esses esforços, deve-se voltar à formulação dos elementos de membrana e de placa.

Maiores informações sobre o programa podem ser encontradas na biblioteca de softwares do departamento de Estruturas da Escola de Engenharia de São Carlos.

Neste capítulo mostra-se alguns exemplos simples, executados com o intuito de verificar a consistência da teoria e do programa elaborado. Os resultados são comparados com soluções teóricas ou de outras modelagens numéricas existentes. Quando se faz a comparação pelo erro percentual, usa-se a relação :

$$
E(\%)=((\text { valor analítico-valor encontado }) / \text { valor analítico }) \times 100
$$

\subsection{EXEMPLOS}

\subsubsection{EXEMPLO}

Neste exemplo é feita uma comparação com modelo apresentado em ZAGOTTIS (1986), onde se estuda o comportamento de uma viga engastada, submetida a um carregamento concentrado na extremidade oposta ao engaste, conforme mostra a figura 5.1. Esse exemplo tem o objetivo de verificar se o programa está operando corretamente o acoplamento e os giros das matrizes de rigidez dos elementos envolvidos. Apesar da sua simplicidade, trata-se de um eficiente teste para se verificar a confiabilidade de uma rotina para elementos finitos. 


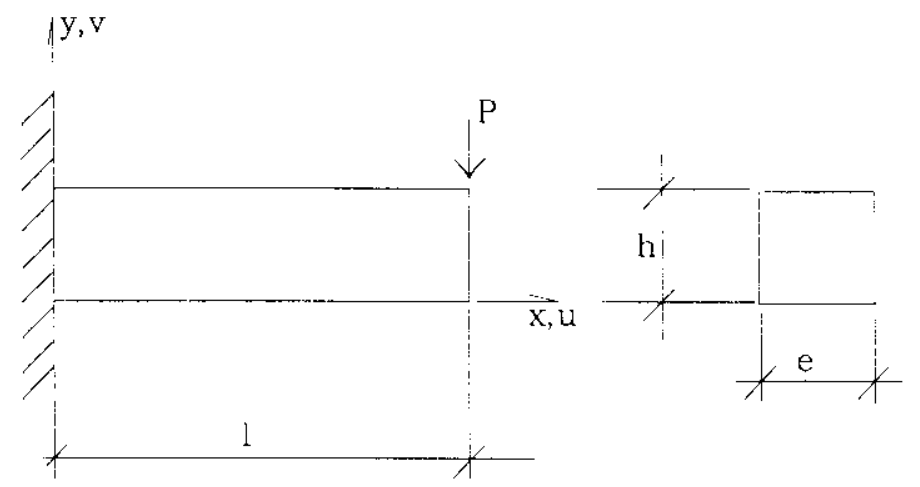

Figura 5.1 Viga engastada com carga concentrada na extremidade.

Considera-se os seguintes valores :

$$
\begin{array}{lll}
P=12.0 \mathrm{tf} & I=2.0 \mathrm{~m} & \mathrm{~h}=1.0 \mathrm{~m} \\
e=1.0 \mathrm{~m} & v=0.0 & E=1000.00 \mathrm{tf} / \mathrm{m}^{2}
\end{array}
$$

Com o intuito de verificar a correta formulação teórica, executa-se o exemplo em uma posição localizada no espaço, como mostra a figura 5.2. A malha usada é idêntica em ZAGOTIS (1986), para se comparar os valores dos deslocamentos encontrados onde também se usa o elemento CST.

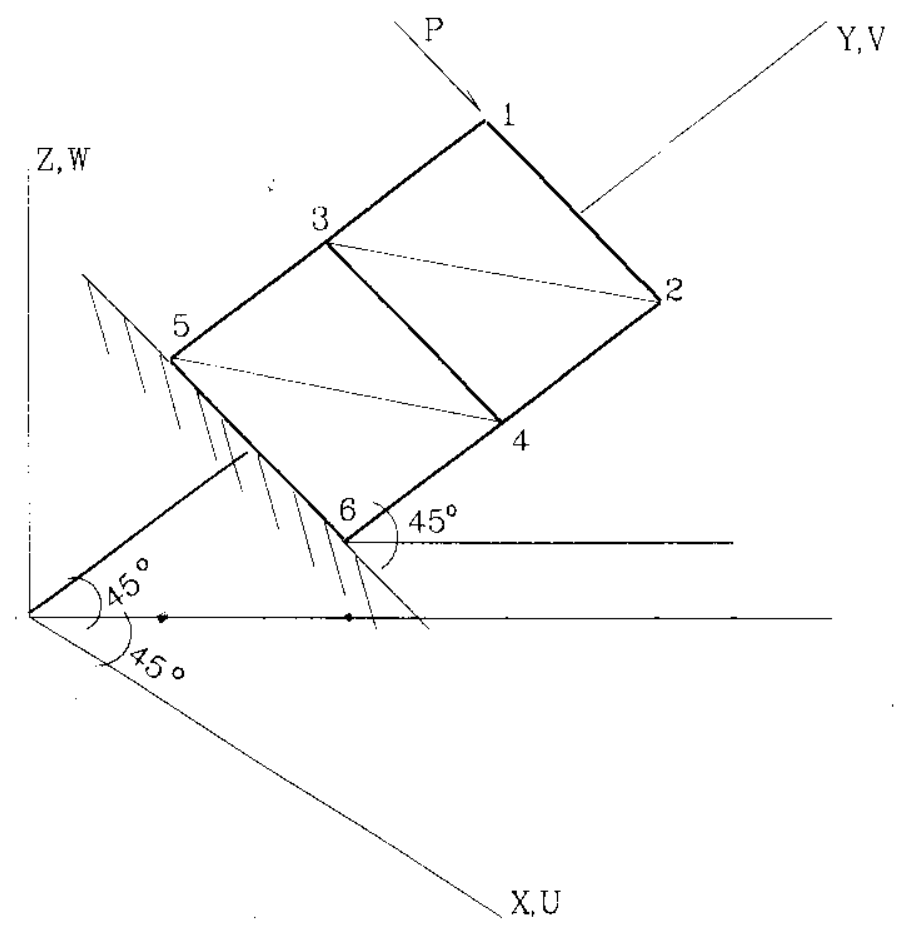

Figura 5.2 Viga engastada inclinada no espaço. 
Os deslocamentos nodais são mostrados em seguida, lembrando que são dados segundo a direção das coordenadas globais . Fazendo-se as devidas projeções ( nas direções 21 e 51), verifica-se a completa igualdade entre os valores encontrados em ZAGOTIS (1986).

$\begin{array}{lcccccc}\text { NO } & \text { Ux } & \text { Vy } & \text { Wz } & \theta x & \theta y & \theta z \\ & & & & & & \\ 1 & .86722 \mathrm{E}-01 & .86722 \mathrm{E}-01 & -.74006 \mathrm{E}-01 & .44637 \mathrm{E}-16 & .44637 \mathrm{E}-16 & .72739 \mathrm{E}-16 \\ 2 & .45154 \mathrm{E}-01 & .45154 \mathrm{E}-01 & -.11250 \mathrm{E}+00 & .38178 \mathrm{E}-16 & .42643 \mathrm{E}-16 & -.79930 \mathrm{E}-16 \\ 3 & .37855 \mathrm{E}-01 & .37855 \mathrm{E}-01 & -.18545 \mathrm{E}-01 & .47038 \mathrm{E}-16 & .47331 \mathrm{E}-16 & -.65084 \mathrm{E}-16 \\ 4 & .10887 \mathrm{E}-01 & .10887 \mathrm{E}-01 & -.50387 \mathrm{E}-01 & .44329 \mathrm{E}-16 & .44329 \mathrm{E}-16 & -.78888 \mathrm{E}-16 \\ 5 & .00000 \mathrm{E}+00 & .00000 \mathrm{E}+00 & .0000 \mathrm{E}+00 & .00000 \mathrm{E}+00 & .00000 \mathrm{E}+00 & .00000 \mathrm{E}+00 \\ 6 & .00000 \mathrm{E}+00 & .00000 \mathrm{E}+00 & .0000 \mathrm{E}+00 & .00000 \mathrm{E}+00 & .00000 \mathrm{E}+00 & .00000 \mathrm{E}+00\end{array}$

Tabela 5.1 Deslocamentos na viga engastada (sistema global).

\subsubsection{EXEMPLO.}

Partindo do exemplo anterior, executa-se a mesma viga com refinamento melhor na malha, conforme figura 5.3. O resultado é comparado com a teoria de viga conhecida, para deflexão vertical no ponto $\mathrm{C}$.

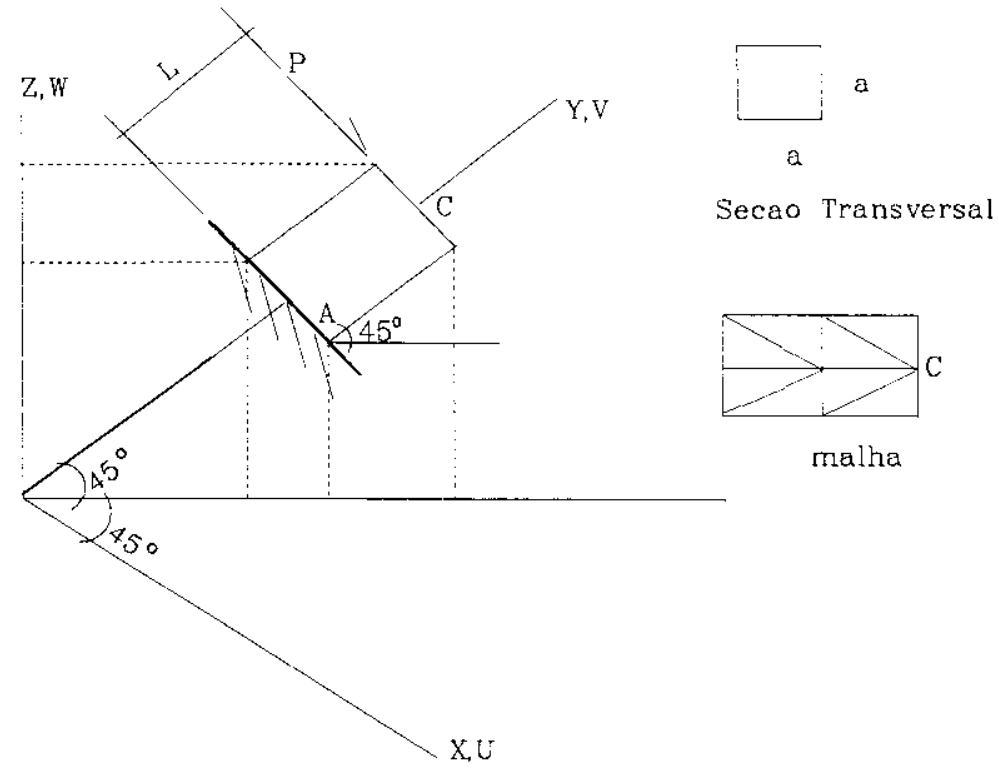

Figura 5.3 Viga engastada inclinada no espaço. 
Para esse exemplo, usou-se as seguintes grandezas físicas :

$$
L=2.0 \mathrm{~m} ; \quad a=1.0 \mathrm{~m} \quad P=1.0 \mathrm{tf} \quad E \mathrm{I}=1.0 \mathrm{tf} . \mathrm{m}^{2}
$$

$\mathrm{Na}$ figura 5.4 mostra-se o comportamento do modelo para a análise do deslocamento no ponto $C$ ( considerando-se a direção paralela à da carga $P$ ), e as tensão atuante no ponto $A$. As malhas executadas correspondem a $9,25,49$ e 81 pontos nodais

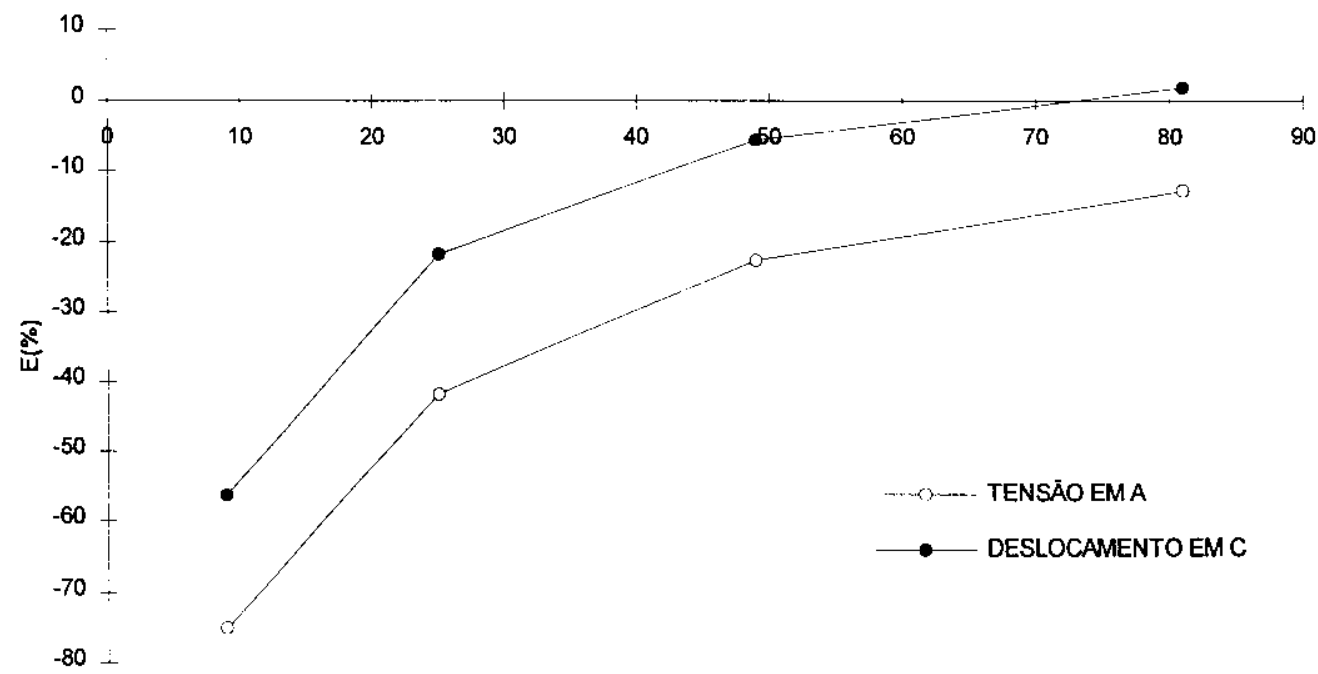

Figura 5.4 Comportamento do modelo para viga engastada.

Nesse exemplo, pela convergência verificada, confirma-se o perfeito comportamento das rotações executadas.

\subsubsection{EXEMPLO.}

Nesse exemplo, procura-se verificar se o programa está operando corretamente as ações devido aos efeitos da placa. Executa-se uma placa 
inclinada no espaço, com carregamento uniformemente distribuido, perpendicular ao seu plano médio. Considera-se a placa engastada nas bordas. Os resultados numéricos do deslocamento perpendicular ao plano médio do ponto $\mathrm{C}$, e dos momentos $\mathrm{Mx}$ e $\mathrm{My}$, são comparados com resultados dados por TIMOSHENKO (1959).

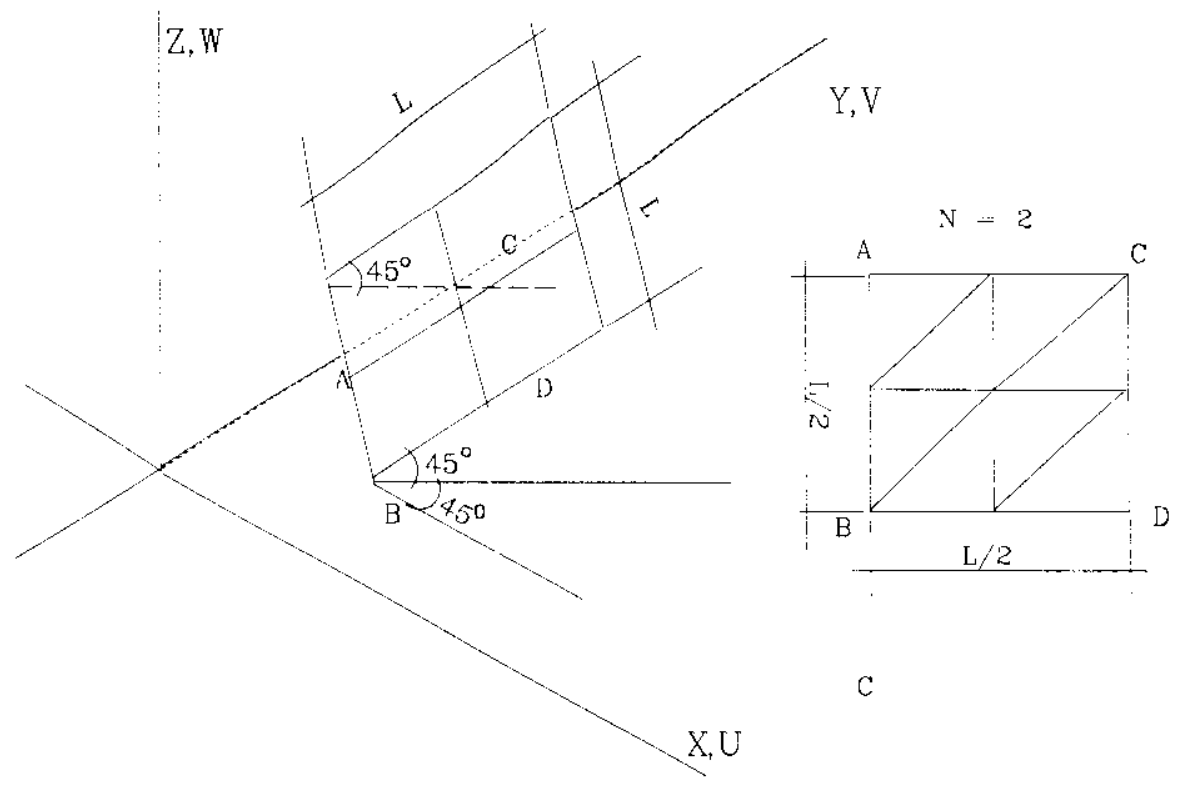

Figura 5.5 Placa inclinada no espaço engastada na quatro bordas.

$\mathrm{Na}$ figura 5.6 mostra-se o comportamento do modelo. As malhas executadas correspondem a $9,25,49$ e 81 pontos nodais. 


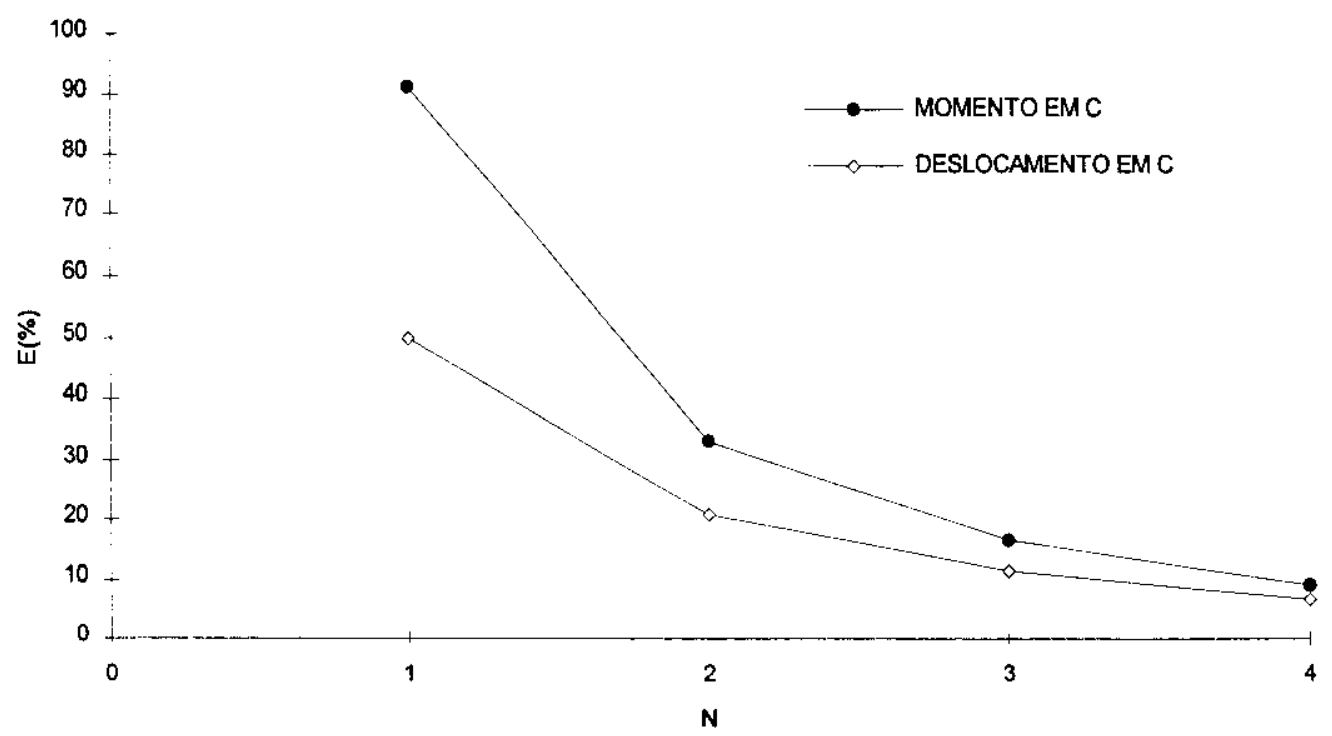

Figura 5.6 Comportamento da placa inclinada no espaço.

Da mesma forma que no exemplo 5.2.2, verifica-se que o elemento de placa DKT responde corretamente ao acoplamento realizado. Isso é observado, também, pela convergência obtida na figura 5.6.

\subsubsection{EXEMPLO}

Neste exemplo verifica-se o comportamento do elemento finito de casca plano adotado, para a cobertura cilíndrica dada na figura 5.6. A orientação das malhas está mostrada na figura 5.7. As malhas executadas cor-

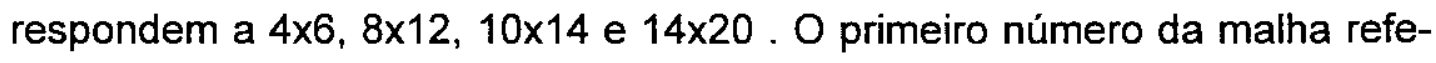
re-se às divisões no sentido angular, e o segundo às divisões no sentido longitudinal. Neste caso, por causa da simetria, executou-se apenas $1 / 4$ da casca dada, como pode ser observado na figura 5.7 


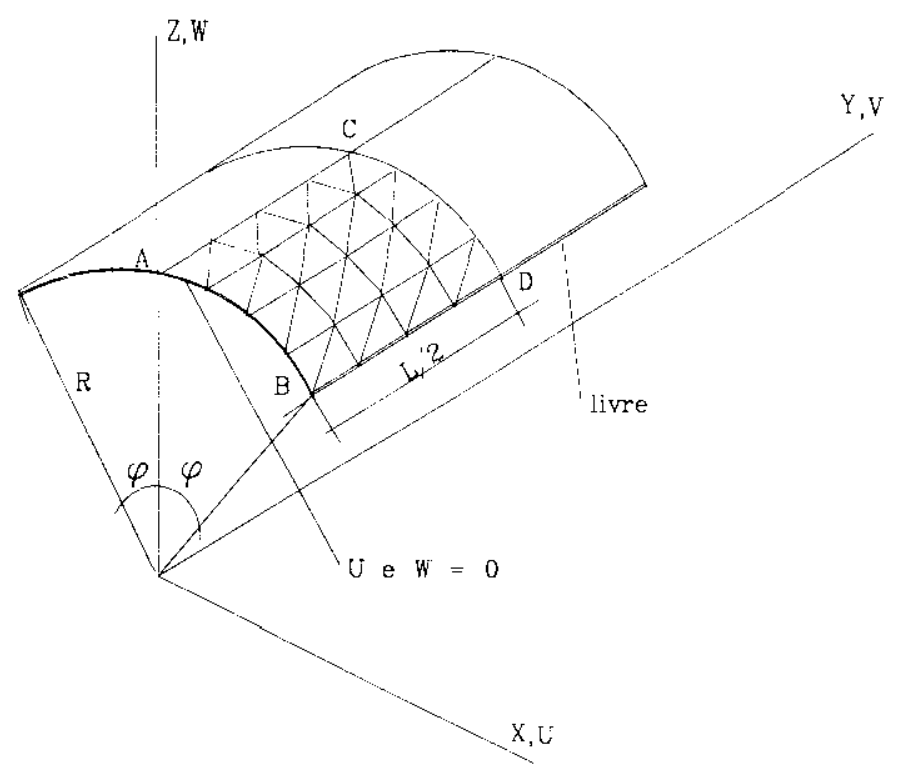

Figura 5.7 Cobertura em casca cilíndrica.

As grandezas físicas adotadas nesse exemplo são :

$$
\begin{array}{lll}
E=3.0 \times 10^{6} \mathrm{psi} & \mathrm{L}=50 \mathrm{ft} & \text { espessura }=3 \text { in } \\
P=90 \mathrm{lb} / \mathrm{ft}^{2} & v=0.0 & \varphi=40^{\circ} \quad \mathrm{R}=25 \mathrm{ft}
\end{array}
$$

Nas figuras 5.8, 5.9, 5.10 e 5.11 mostra-se comportamento do modelo para a execução da malha $14 \times 20$. Os resultados são comparados com a solução analítica dada em ZIENKIEWICS (1977) e COOK (1989). 


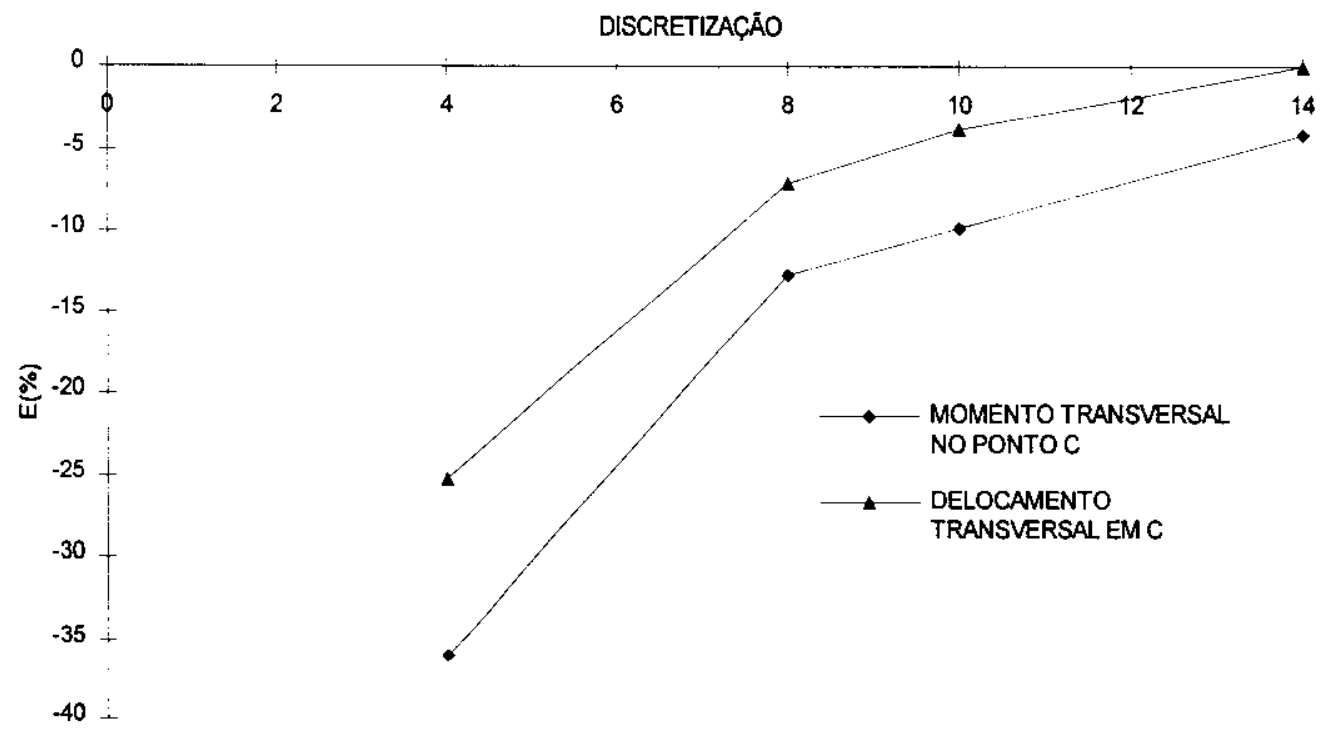

Figura 5.8 Comportamento do modelo para deslocamento e momento transversal no ponto $\mathrm{C}$.

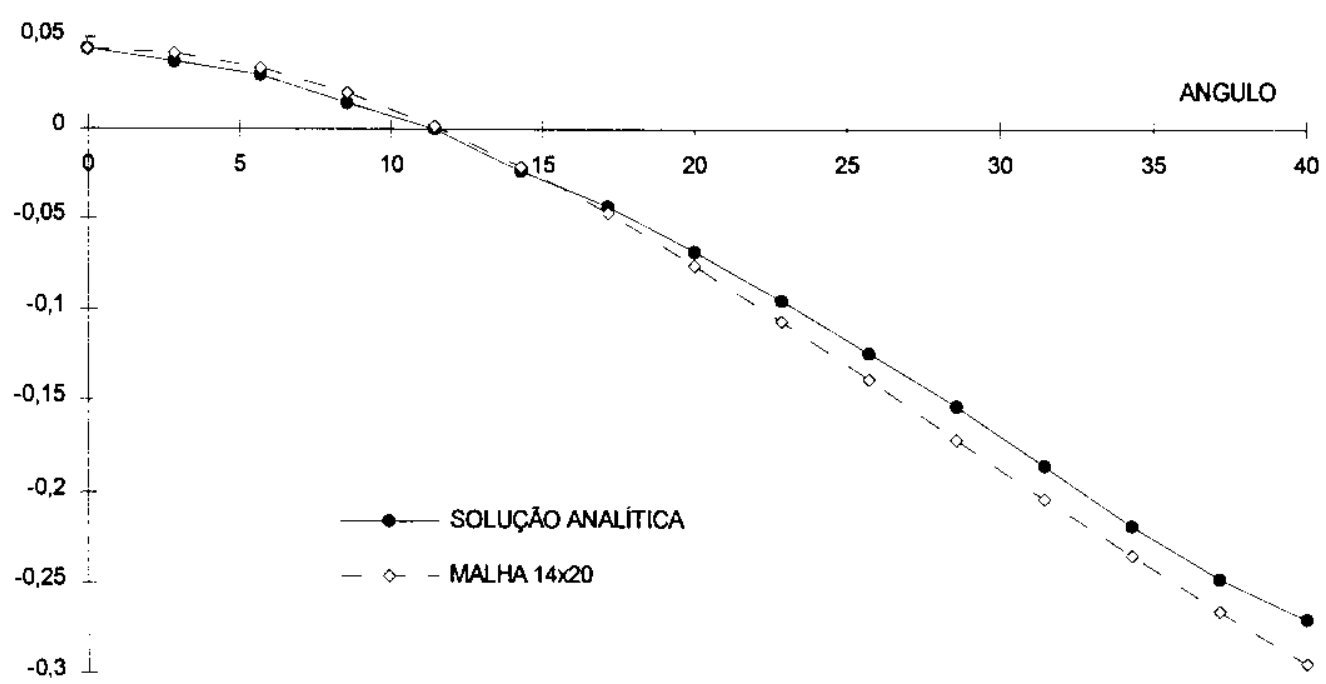

Figura 5.9 Comparação entre a solução analítica e a execução da malha $14 \times 20$, do deslocamento transversal na seção central. 


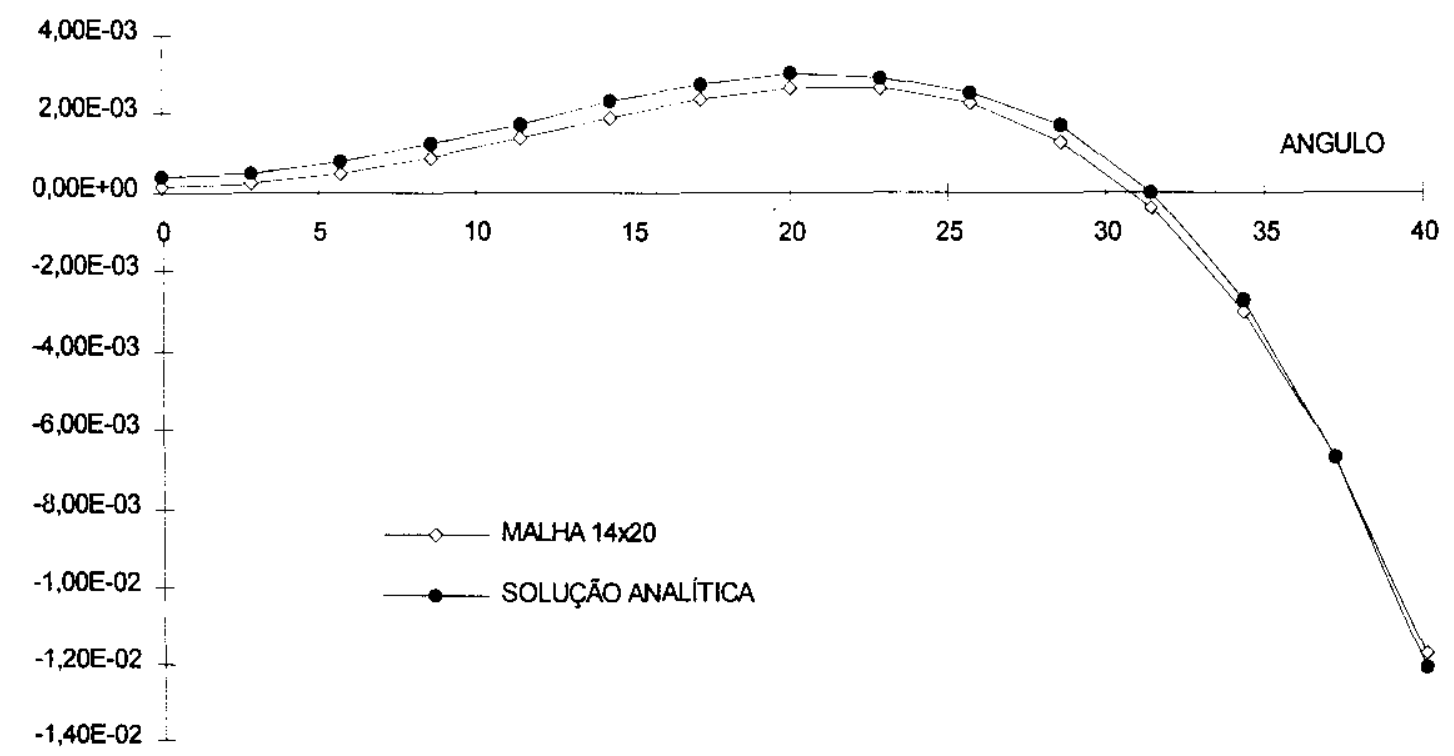

Figura 5.10 Comparação entre a solução analítica e a execução da malha $14 \times 20$ para o deslocamento longitudinal no apoio.

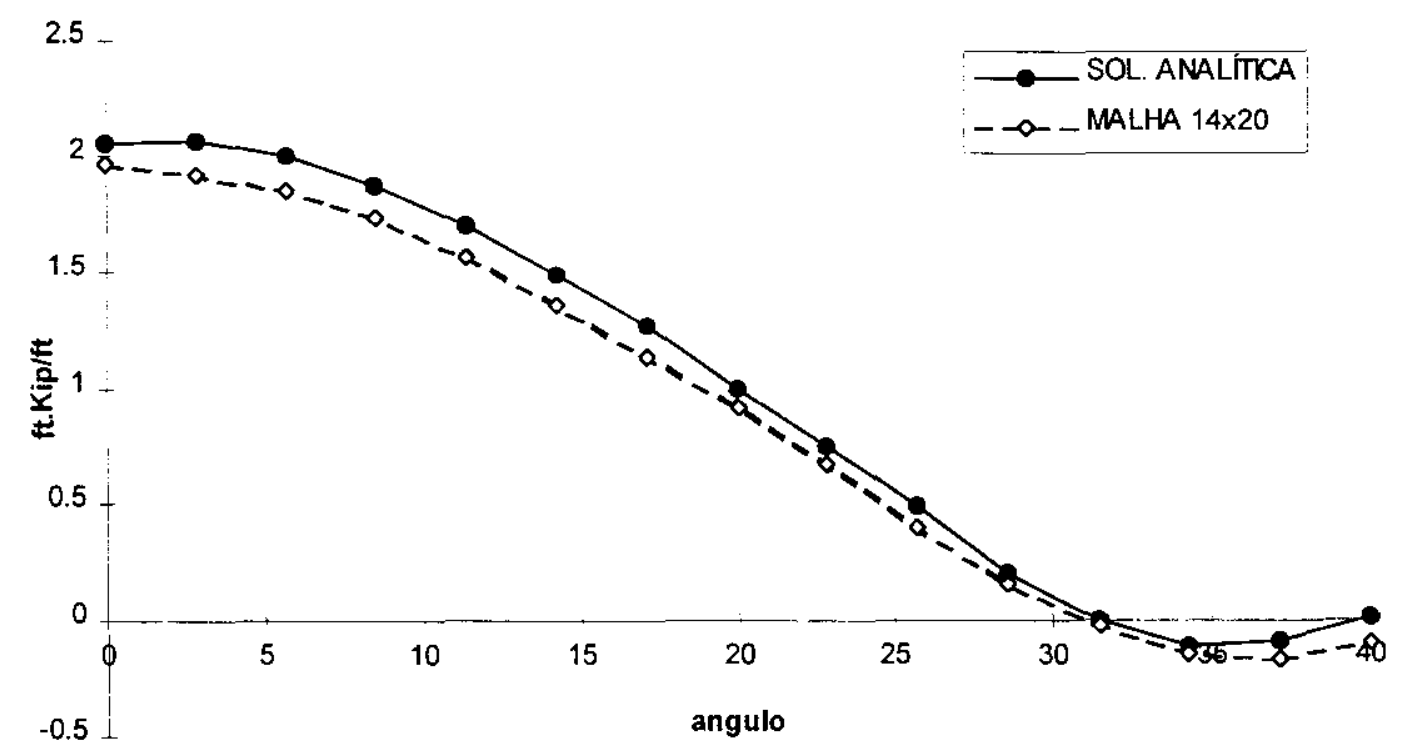

Figura 5.11 Comparação entre a solução analítica e a malha 14×20 do momento transversal na seção central. 
Pelos resultados, observa-se que esse elemento fornece bons resultados para exemplos para cascas abatidas, onde o efeito de flexão é predominante

Como o elemento de membrana usado foi o CST, sendo um elemento pobre para problemas com efeitos de membrana, acredita-se que, em cascas onde tais efeitos sejam relevantes, para se obter bons resultados necessita-se de bom refinamento da malha. Esse elemento de casca não foi testado para cascas onde o efeito de membrana é predominante 


\section{CONCLUSÃO}

Neste trabalho foi desenvolvido um elemento finito de casca formado pelo acoplamento do elemento de placa DKT(Discrete Kirchhoff Teory), e do elemento de membrana CST( Constant Strain Triangle).

Esse elemento fornece boas respostas para cascas em que o efeito de flexão sejam predominantes, o que pode ser observado no exemplo executado.

Considerando-se o fato de se usar o elemento finito de membrana CST, de fraca convergência, acredita-se que quando houver predominância dos efeitos de membrana, também necessitar-se-á de bom refino da malha para se obter bons resultados. Esse elemento não foi testado em casos que há essa predominância.

Como possibilidade de prosseguimento do trabalho, sugere-se a busca de um elemento que represente melhor os efeitos de membrana. Podese conseguir resultados melhores com o elemento LST, entretanto pelo fato desse elemento possuir nós intermediários, a semi-banda do sistema de equações atinge valores elevados, ocupando maior espaço na memória volátil, e absorvendo maior tempo de execução do programa.

Em IBRAHIMBEGOVIC (1990), e ALLMAN(1993), encontram-se alternativas que proporcionam melhoria no comportamento da membrana. $A$ 
verificação de tais resultados, é deixada como sugestão de prosseguimento do trabalho aqui iniciado.

Outra dificuldade encontrada ao longo do trabalho é relativa ao modo de entrada de dados. É comum encontrar-se em literatura corrente geradores de malhas para elementos planos. Quando se trata de elementos espaciais, não se verifica a mesma facilidade.

A montagem do arquivo de entrada de dados, quando se pretende alcançar um bom refinamento da malha, é extremamente extenuante e muito imprecisa. O tempo gasto nessa etapa do trabalho, poderia ser utilizado de forma mais proveitosa, na análise dos resultados obtidos. Sugere-se também um trabalho que possa sanar essa dificuldade. 


\section{BIBLIOGRAFIA}

ARGIRYS, J.H.(1950) Energy Theorems and Structural Analysis. Aircraft Engineering. 26. Pp 347-356, 383-387, 3941954.

ALLMAN, D.J. (1993). Variational validation of a membrane finite element with drilling rotations. Communications in Numerical Methods in Engineering, v.9, n.4, p.345-351, Apr.

BATOZ, J. L. ; BATHE, K-J.; HO, L-W. (1980). A study of three-node triangular plate bending elements. International Journal for Numerical Methods in Engineering, v.15, p.1771-1812.

BATOZ, J. L.; LARDEUR, P. (1989). Discrete shear triangular nine D.O.F. element for the analysis of thick to very thin plates. International Journal for Numerical Methods in Engineering, v.28, n.3, p.533-560, Mar.

BAZELEY, G.P. et al. (1965). Triangular elements in plate bendingconforming and non conforming solutions. In: CONF. ON MATRIX METHODS IN STRUCTURAL MECHANICS, WPAFB, Ohio. Proc. p.547576.

BILLINGTON, D. P. (1982). Thin shell concrete structures. New York, McGraw-Hill. 
CLOUGH, R.W. (1960). The finite element in plane stress analysis. In: A.S.C.E. CONF. ON ELETRONIC COMPUTATION, 2nd, Pittsburgh, USA, Sept. Proc.

CLOUGH, R.W.; FELIPPA, C.A. (1965). A refined quadrilateral element for analysis of plate bending. In: CONF. ON MATRIX METHODS IN STRUCTURAL MECHANICS, WPAFB, Ohio. Proc. p.399-440.

CLOUGH, R.W.; JOHNSON, C.P. (1968). A finite element aproximation for the analysis for the thin shells. Int. J. Solids Structures, v. 4, p.43-60.

CLOUGH, R.W.; TOCHER, J. L. (1965). Finite element stiffness for analysis of plates bending. In: CONF. ON MATRIX METHODS IN STRUCTURAL MECHANICS, WPAFB, Ohio. Proc. p.515-545.

CODA, H.B. (1993). Análise tridimensional transiente de estruturas pela combinação entre o método dos elementos de contorno e o método dos elementos finitos. Tese (Doutorado) - Escola de Engenharia de São Carlos, Universidade de São Paulo.

COOK, R.D.; MALKUS, D.S.; PLESHA, M.E. (1989). Concepts and applications of finite element analysis. 3.ed. New York, John Wiley \& Sons.

COURANT, R. (1943). Variational methods for the solution of problems of equilibrium and vibration. Bulletin of the American Mathematical Society, v.49, p.1-43. 
DHATT,G.; MARCOTTE, L.; MATTE, Y. (1986). A new triangular discrete Kirchhoff plate/shell element. International Journal for Numerical Methods In Engineering, v.23, p.453-470.

FLUGGE, W. (1960). Stresses in shells. Berlin, Springer-Verlag.

HO, P.T.S. (1992). Comparison of performance of a flat faceted shell element and a degenerated superparametric shell element. Computers and Structures, v.44, n.4, p.895-904, Aug.

IBRAHIMBEGOVIC, A.; TAYLOR, R.L.; WILSON, E.L. (1990). Robust quadrilateral membrane finite element with drilling degrees of freedom. International Journal for Numerical Methods in Engineering, v.30, n.3, p.445-457, Aug.

IRONS, B.M. (1966). Engineering application of numerical integration in stiffness method. A.I.A.A. Journal, v.14, p.2035-7.

IRONS, B.M. (1966). Numerical integration applied to finite element methods. In: CONF. USE OF DIGITAL COMPUTERS IN STRUCTURES ENG., Univ. Of Newcastle. Proc.

PETROLITO, J. (1989). Modified ACM element for thick plate analysis. Computers and Structures, v.32, n.6, p.1303-1309.

PHAAL, R.; CALLADINE, C. R. (1992). Simple class of finite elements for plate and shell problems - I: Elements for beams and thin flat plates. International Joumal for Numerical Methods in Engineering, v.35, n.5, p.955-977, Sep.

PHAAL, R.; CALLADINE, C.R. (1992). Simple class of finite elements for plate and shell problems - II: An element for thin shells, with only translational degrees of fridoom. International Joumal for Numerical Methods in Engineering, v.35, n.5, p.979-996, Sep. 
REISSNER, E. (1945). The effect of transverse shear deformation on the bending of elastic plates. Journal of Applied Mechanics, v.12, p.69-77.

SALMON, D.C.; ABEL, J.F. (1989). Assessing the effect of shape distortion in Q9 membrane elements. Computers and Structures, v.33, n.5, p.11831190.

SZE, K.Y.; CHOW, C.L. (1991). Mixed formulation of a four-node Mindlin shell/plate with interpolated covariant transverse shear strains. Computers and Structures, v.40, n.3, p.775-784.

TAIG, I.C. (1961). Structural analysis by the matrix displacement method. Engl. Eletric Aviation Report No S017.

TIMOSHENKO, S.P.; GOODIER, J.N. (1951). Theory of elasticity. New York, McGraw-Hill.

TIMOSHENKO, S.P.; WOINOWSKY-KRIEGER, S. (1959). Theory of plates and shells. New York, McGraw-Hill.

TURNER, M. et al. (1956). Stiffness and deflection analysis of complex structures. Journal of Aeronautical Science, v.23, p.805-823.

WILSON, E.L. (1974). The static condesation algorithn. Int. J. Num. Meth. Eng., v.8, n.1, p.198-203, Jan.

YUAN, K.-Y.; HUANG, Y.-S.; PIAN, T.H.H. (1993). New strategy for assumed stresses for 4-node hybrid stress membrane element. International Joumal for Numerical Methods in Engineering, v.36, n.10, p.1747-1763, May. 
ZAGOTTIS, D.L. (1986). Introdução à teoria das estruturas: elasticidade elementos finitos. São Paulo, EPUSP. cap.10

ZIENKIEWICZ, O.C. (1977). The finite element method. 3.ed. London, McGraw-Hill, London. 